\title{
Low-mass low-metallicity AGB stars as an efficient $i$-process site explaining CEMP-rs stars ${ }^{\star, \star \star}$
}

\author{
D. Karinkuzhi ${ }^{1,2,3}$, S. Van Eck ${ }^{2}$, S. Goriely ${ }^{2}$, L. Siess ${ }^{2}$, A. Jorissen ${ }^{2}$, T. Merle ${ }^{2}$, A. Escorza ${ }^{2,4}$, and T. Masseron ${ }^{5,6}$ \\ 1 Department of Physics, Indian Institute of Science, Bangalore 560012, India \\ 2 Institut d'Astronomie et d'Astrophysique, Université Libre de Bruxelles, ULB, Campus Plaine C.P. 226, Boulevard du Triomphe, \\ 1050 Bruxelles, Belgium \\ e-mail: dkarinku@ulb.ac.be, svaneck@astro.ulb.ac.be \\ 3 Department of Physics, Jnana Bharathi Campus, Bangalore University, Bangalore 560056, India \\ ${ }^{4}$ Institute of Astronomy, KU Leuven, Celestijnenlaan 200D, 3001 Leuven, Belgium \\ 5 Instituto de Astrofísica de Canarias, 38205 La Laguna, Tenerife, Spain \\ ${ }^{6}$ Departamento de Astrofísica, Universidad de La Laguna, 38206 La Laguna, Tenerife, Spain
}

Received 10 July 2020 / Accepted 22 October 2020

\begin{abstract}
Context. Among carbon-enhanced metal-poor (CEMP) stars, some are found to be enriched in slow-neutron capture ( $s$-process) elements (and are then tagged CEMP-s), some have overabundances in rapid-neutron capture ( $r$-process) elements (tagged CEMP-r), and some are characterized by both $s$ - and $r$-process enrichments (tagged CEMP-rs). The current distinction between CEMP-s and CEMP-rs is based on their $[\mathrm{Ba} / \mathrm{Fe}]$ and $[\mathrm{Eu} / \mathrm{Fe}]$ ratios, since barium and europium are predominantly produced by the $s$ - and the $r$ process, respectively. The origin of the abundance differences between CEMP-s and CEMP-rs stars is presently unknown. It has been claimed that the $i$-process, whose site still remains to be identified, could better reproduce CEMP-rs abundances than the $s$-process. Aims. We propose a more robust classification method for CEMP-s and CEMP-rs stars using additional heavy elements other than Ba and Eu. Once a secure classification is available, it should then be possible to assess whether the $i$-process or a variant of the $s$-process better fits the peculiar abundance patterns of CEMP-rs stars.

Methods. We analyse high-resolution spectra of 24 CEMP stars and one $r$-process enriched star without carbon-enrichment, observed mainly with the high-resolution HERMES spectrograph mounted on the Mercator telescope (La Palma) and also with the UVES spectrograph on VLT (ESO Chile) and HIRES spectrograph on KECK (Hawaii). Stellar parameters and abundances are derived using MARCS model atmospheres. Elemental abundances are computed through spectral synthesis using the TURBOSPECTRUM radiative transfer code. Stars are re-classified as CEMP-s or -rs according to a new classification scheme using eight heavy element abundances. Results. Within our sample of 25 objects, the literature classification is globally confirmed, except for HE 1429-0551 and HE 2144-1832, previously classified as CEMP-rs and now as CEMP-s stars. The abundance profiles of CEMP-s and CEMP-rs stars are compared in detail, and no clear separation is found between the two groups; it seems instead that there is an abundance continuum between the two stellar classes. There is an even larger binarity rate among CEMP-rs stars than among CEMP-s stars, indicating that CEMP-rs stars are extrinsic stars as well. The second peak s-process elements $(\mathrm{Ba}, \mathrm{La}, \mathrm{Ce})$ are slightly enhanced in CEMP-rs stars with respect to first-peak $s$-process elements ( $\mathrm{Sr}, \mathrm{Y}, \mathrm{Zr}$ ), when compared to CEMP-s stars. Models of radiative $s$-process nucleosynthesis during the interpulse phases reproduce well the abundance profiles of CEMP-s stars, whereas those of CEMP-rs stars are explained well by low-metallicity $1 M_{\odot}$ models experiencing proton ingestion. The global fitting of our $i$-process models to CEMP-rs stars is as good as the one of our $s$-process models to CEMP-s stars. Stellar evolutionary tracks of an enhanced carbon composition (consistent with our abundance determinations) are necessary to explain the position of CEMP-s and CEMP-rs stars in the Hertzsprung-Russell diagram using Gaia DR2 parallaxes; they are found to lie mostly on the red giant branch (RGB).

Conclusions. CEMP-rs stars present most of the characteristics of extrinsic stars such as CEMP-s, $\mathrm{CH}$, barium, and extrinsic S stars; they can be explained as being polluted by a low-mass, low-metallicity thermally-pulsing asymptotic giant branch (TP-AGB) companion experiencing $i$-process nucleosynthesis after proton ingestion during its first convective thermal pulses. As such, they could be renamed CEMP-sr stars, since they represent a particular manifestation of the $s$-process at low-metallicities. For these objects a call for an exotic $i$-process site may not necessarily be required anymore.
\end{abstract}

Key words. nuclear reactions, nucleosynthesis, abundances - stars: AGB and post-AGB - binaries: spectroscopic stars: fundamental parameters

\section{Introduction}

Low- and intermediate-mass stars (LIMS) dominate the stellar populations in galaxies. They are the siege of a rich nucle-

* Full Tables A.1 and B.2 are only available at the CDS via anonymous ftp to cdsarc.u-strasbg.fr (130.79.128.5) or via http: //cdsarc.u-strasbg.fr/viz-bin/cat/J/A+A/645/A61

$\star \star$ This paper is partly based on observations made with the ESO/VLT, at Paranal Observatory, under program 69.D-0063. osynthesis which produces most of the elements heavier than iron, and thus play a crucial role in the chemical evolution of galaxies. The dominant nucleosynthetic processes are the slow neutron-capture process ( $s$-process) and the rapid neutroncapture process ( $r$-process). Detailed spectroscopic analyses of stars enhanced in heavy elements constitute an important method for investigating the physical and chemical conditions required for these processes to operate. While the nuclear mechanisms responsible for the $s$ - and $r$-processes are clearly identified, the 
origin of the peculiar abundance patterns in the so-called rs-stars showing enhancements of both $s$-process and $r$-process elements is still an open question (Barbuy et al. 1997; Hill et al. 2000; Beers \& Christlieb 2005; Jonsell et al. 2006; Masseron et al. 2010; Gull et al. 2018, and references therein).

Stars enriched in both $r$ - and $s$-elements are also carbonenriched and are mostly found among metal-poor stars; they are thus tagged CEMP-rs (CEMP stands for carbon-enhanced metal-poor) stars (e.g. Beers \& Christlieb 2005; Masseron et al. 2010) but also among CH stars. Keenan (1942) coined the term "CH stars" to refer to high-velocity carbon stars that exhibit very strong $\mathrm{G}$ bands due to the $\mathrm{CH}$ molecule and otherwise weak metallic lines. Both CEMP-s and CEMP-rs stars seem to belong to binary systems (Lucatello et al. 2005; Starkenburg et al. 2014; Hansen et al. 2016a). The current criterion used to distinguish CEMP-rs from CEMP-s stars is based on $[\mathrm{Ba} / \mathrm{Eu}]$ or $[\mathrm{La} / \mathrm{Eu}]$ ratio (Beers \& Christlieb 2005; Masseron et al. 2010). However, this classification could probably be more robust, and it is one of the goals of this paper to devise a better classification criterion.

The scenarios proposed to explain CEMP-rs overabundances (Jonsell et al. 2006; Masseron et al. 2010; Hampel et al. 2016) involve a primordial origin (pollution of the birth cloud by an $r$-process source); pollution of the binary by a third massive star (triple system); pollution by the primary exploding as a type 1.5 supernova or accretion-induced collapse; or pollution by the intermediate-process or $i$-process, i.e. a process leading to neutron densities of the order of $N_{n} \sim 10^{14-15} \mathrm{~cm}^{-3}$, intermediate between those of the $s$-process $\left(N_{n} \sim 10^{8} \mathrm{~cm}^{-3}\right)$ and those required by the $r$-process $\left(N_{n} \gg 10^{20} \mathrm{~cm}^{-3}\right)$. This process could also explain why low-metallicity stars, including postasymptotic giant branch (post-AGB) stars, show lower abundance in lead and other heavy-s nuclei with respect to what is expected from standard $s$-process model predictions. More precisely, below $[\mathrm{Fe} / \mathrm{H}] \sim-0.7$, a very wide range of neutron irradiations is needed to explain the spread in heavy-s abundances measured (Lugaro et al. 2015; De Smedt et al. 2016). These authors suggested that the $i$-process could meet this challenge. While one-zone parametric simulations of the $i$-process tend to reproduce convincingly the abundances in CEMP-rs stars (Hampel et al. 2016), its astrophysical site has not yet been identified. A number of suggestions have been proposed, among which the proton ingestion in C-rich layers heated to relatively high temperatures, such as during core He flash in very low-metallicity low-mass stars (e.g. Campbell et al. 2010), during the thermal pulse phase of intermediate-mass or massive AGB (super-AGB) stars of low metallicity (e.g. Goriely \& Siess 2005; Jones et al. 2016), during the post-AGB phase ("final thermal pulse") (e.g. Herwig et al. 2011), during rapid accretion of H-rich material on WDs (e.g. Denissenkov et al. 2017, 2018), or during shell He burning in massive very low-metallicity Pop II or Pop III stars (e.g. Clarkson et al. 2018). Another possibility for the $i$-process operation would be the ingestion of protons in the He-burning convective region of low-metallicity low-mass AGB stars, as first described by Cowan \& Rose (1977) and Iwamoto et al. (2004). This specific astrophysical site is the one we focus on in the present study. We note, however, that our recent identification and analysis (Karinkuzhi et al. 2018) of a star enriched in $s$ - and $r$-elements at comparatively high metallicity $([\mathrm{Fe} / \mathrm{H}] \sim-0.7)$ puts a strong constraint on the occurrence of the $i$-process, which may not be limited to very low-metallicity environments.

The paper is structured as follows. The sample of CEMP-s and CEMP-rs stars and their binarity are described in Sect. 2, while the stellar parameter and abundance determinations are derived in Sects. 3 and 4. The new methodology to classify
CEMP-s and -rs stars is presented in Sect. 5. The abundances of the programme stars are compared to literature determinations in Sect. 6 and are to nucleosynthesis computations in Sect. 7. Section 8 focuses on the discussion of specific abundance ratios concerning CNO and heavy elements. Finally, the stars are located in the Hertzsprung-Russell (HR) diagram in Sect. 9 using Gaia DR2 parallaxes, and conclusions are drawn in Sect. 10.

\section{Stellar sample and observations}

We analysed a sample of 25 metal-poor stars, 24 of which are carbon-enriched and one an $r$-process-enriched star without carbon enrichment. They were selected from the catalogue of carbon stars from the Hamburg-ESO survey by Christlieb et al. (2001) and HK survey by Beers et al. (1992), as well as from CEMP and $\mathrm{CH}$ stars in the literature, as listed in Table 1, which includes 15 CEMP-s and -rs stars already analysed in the literature. Therefore, we re-analysed these 15 stars and we present the first complete abundance pattern for ten new objects. Furthermore, many of the stars listed in Table 1, already classified as CEMP-s, in fact had no measured abundance for either $\mathrm{Eu}$ or any other $r$ process elements; their classification is therefore purely based on $s$-process elements, and their possible rs nature would therefore remain undetected. It was the aim of our analysis to derive these missing abundances so as to decide whether these stars are CEMPs or -rs. Column 8 of Table 1, "Orig. class", lists the classification using our newly derived abundances and the $[\mathrm{La} / \mathrm{Eu}]$ criterion of Beers \& Christlieb (2005) as described in Sect. 5.

High-resolution spectra for most of the objects were acquired from the HERMES spectrograph (Raskin et al. 2011) mounted on the $1.2 \mathrm{~m}$ Mercator telescope at the Roque de los Muchachos Observatory, La Palma, Canary Islands, which covers the wavelength range 3800-9000 $\AA$ at a resolution $R \sim 86000$. These objects were observed as part of a long-term monitoring of radial velocities to detect the binary nature and derive orbital parameters of specific families of stars (Gorlova et al. 2014). Spectra observed on different nights were co-added after correcting for the Doppler shifts to maximize the signal-to-noise ratio $(\mathrm{S} / \mathrm{N})$. We also used a few spectra obtained with the UVES spectrograph mounted on the ESO Very Large Telescope with a wavelength coverage from 3300 to $6700 \AA$. Three stars were taken from the HIRES/KECK data archive and have wavelength coverage from 3700 to $8000 \AA$.

Information about radial-velocity variability is provided in the "Bin" column in Table 1. For many stars information was already available in the literature, but for five objects additional radial velocities and/or new orbits will be published in a forthcoming paper. Table 1 reveals the high incidence of binarity among CEMP-rs objects; according to our new classification scheme (see Sect. 5), 9 out of 11 CEMP-rs stars (82\%) have been detected as binaries, compared to 6-9 out of 13 CEMP-s stars (46-69\%). Thus, the binarity rate of CEMP-rs stars is reminiscent of the value found in extrinsic-star classes, like extrinsic $\mathrm{S}$ stars, barium stars, $\mathrm{CH}$ stars, and their lower metallicity counterparts, the CEMP-s stars.

\section{Derivation of the atmospheric parameters}

Our programme stars are low-metallicity carbon stars with strong molecular bands throughout their spectra. It is therefore difficult to find unblended neutral and ionized $\mathrm{Fe}$ lines in their spectrum. Adopting the usual excitation and ionization 
Table 1. Programme stars.

\begin{tabular}{|c|c|c|c|c|c|c|c|c|c|c|c|c|}
\hline Name & $\begin{array}{l}T_{\text {eff }} \\
(\mathrm{K})\end{array}$ & $\begin{array}{l}\log g \\
(\operatorname{cgs})\end{array}$ & $\begin{array}{c}\xi \\
\left(\mathrm{km} \mathrm{s}^{-1}\right)\end{array}$ & {$[\mathrm{Fe} / \mathrm{H}]$} & $d_{\mathrm{S}}$ & $d_{\mathrm{rms}}$ & $\begin{array}{l}\text { Orig. } \\
\text { class. }\end{array}$ & $\begin{array}{l}\text { New } \\
\text { class. }\end{array}$ & $\chi^{2}$ & Spec & Bin & Ref. \\
\hline CS 22887-048 & $500 \pm 50$ & $20 \pm 0.15$ & $1.00 \pm 0.05$ & $-2.10 \pm 0.09$ & 0.784 & 0.861 & $\mathrm{~s}$ & $\mathrm{~s}$ & 4.8 & $\mathrm{U}$ & - & - \\
\hline CS 22891-171 & $5215 \pm 68$ & $1.24 \pm 0.09$ & $2.14 \pm 0.14$ & $-2.50 \pm 0.10$ & 0.530 & 0.756 & rs & rs & 5.3 & $\mathrm{U}$ & - & - \\
\hline CS 22942-019 & $5100 \pm 98$ & $2.19 \pm 0.20$ & $1.73 \pm 0.10$ & $-2.50 \pm 0.09$ & 1.084 & 1.192 & s & $\mathrm{s}$ & 9.9 & $\mathrm{U}$ & Y & $\mathrm{d}, \mathrm{g}$ \\
\hline CS 30322-023 & $4500 \pm 100$ & $1.00 \pm 0.50$ & $2.80 \pm 0.10$ & $-3.35 \pm 0.09$ & 0.948 & 1.041 & $\mathrm{~s}$ & $\mathrm{~s}$ & 4.8 & $\mathrm{U}$ & - & - \\
\hline HD 26 & $5169 \pm 108$ & $2.46 \pm 0.18$ & $1.46 \pm 0.08$ & $-0.98 \pm 0.09$ & 1.344 & 1.368 & $\mathrm{~s}$ & $\mathrm{~s}$ & 6.6 & $\mathrm{H}$ & $\mathrm{Y}$ & $\mathrm{b}$ \\
\hline HD 5223 & $4650 \pm 120$ & $1.03 \pm 0.30$ & $2.16 \pm 0.14$ & $-2.00 \pm 0.08$ & 0.471 & 0.654 & rs & rs & 2.2 & $\mathrm{H}$ & $\mathrm{Y}$ & $\mathrm{e}$ \\
\hline HD 55496 & $4642 \pm 39$ & $1.65 \pm 0.14$ & $1.33 \pm 0.08$ & $-2.10 \pm 0.09$ & 1.350 & 1.416 & $\mathrm{~s}$ & $\mathrm{~s}$ & 9.1 & $\mathrm{H}$ & $\mathrm{Y} ?$ & $\mathrm{~g}, \mathrm{~h}$ \\
\hline HD 76396 & $4750 \pm 100$ & $2.00 \pm 0.30$ & $2.00 \pm 0.10$ & $-2.27 \pm 0.10$ & 0.578 & 0.668 & rs & rs & 1.8 & $\mathrm{H}$ & $\mathrm{Y}$ & $\mathrm{b}$ \\
\hline HD 145777 & $4443 \pm 57$ & $0.50 \pm 0.10$ & $2.63 \pm 0.10$ & $-2.32 \pm 0.10$ & 0.448 & 0.575 & rs & rs & 3.9 & $\mathrm{H}$ & Y & $\mathrm{b}, \mathrm{c}$ \\
\hline HD 187861 & $5000 \pm 100$ & $1.50 \pm 0.25$ & $2.00 \pm 0.20$ & $-2.60 \pm 0.10$ & 0.037 & 0.506 & rs & rs & 10.2 & $\mathrm{U}$ & - & - \\
\hline HD 196944 & $5168 \pm 48$ & $1.28 \pm 0.16$ & $1.68 \pm 0.11$ & $-2.50 \pm 0.09$ & 0.438 & 0.547 & 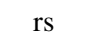 & rs & 1.3 & $\mathrm{U}, \mathrm{H}$ & $\mathrm{Y}$ & $\mathrm{a}$ \\
\hline HD 198269 & $4458 \pm$ & $0.83 \pm 0.08$ & $1.64 \pm 0.09$ & $-2.10 \pm 0.10$ & 0.856 & 0.946 & $\mathrm{~s}$ & $\mathrm{~s}$ & 4 & $\mathrm{H}$ & $\mathrm{Y}$ & $\mathrm{e}$ \\
\hline $\mathrm{HD} 2$ & $5084 \pm$ & $2.18 \pm 0.16$ & $1.60 \pm 0.09$ & $-1.75 \pm$ & 1.106 & 1.1 & $\mathrm{~s}$ & $\mathrm{~s}$ & 3.9 & $\mathrm{H}$ & $\mathrm{Y}$ & $\mathrm{e}$ \\
\hline $\mathrm{HD} 2$ & 4200 & $0.60 \pm 0.20$ & $1.50 \pm$ & $-1.00 \pm 0.10$ & 0.630 & 0.702 & $\mathrm{~s}$ & $\mathrm{~s}$ & 2.7 & $\mathrm{H}$ & $\mathrm{Y}$ ? & g \\
\hline HD 209621 & $4740 \pm 55$ & $1.75 \pm 0.25$ & $1.94 \pm 0.13$ & $-2.00 \pm 0.09$ & 0.314 & 0.517 & rs & rs & 6.3 & $\mathrm{H}$ & $\mathrm{Y}$ & $\mathrm{e}$ \\
\hline HD $221170^{(1)}$ & $4577 \pm 50$ & $0.77 \pm 0.20$ & $1.84 \pm 0.10$ & $-2.40 \pm 0.10$ & 0.101 & 0.208 & $\mathrm{r}$ & $\mathrm{r}$ & - & $\mathrm{H}$ & $\mathrm{N}$ & $\mathrm{i}$ \\
\hline HD 224959 & $4969 \pm 64$ & $1.26 \pm 0.29$ & $1.63 \pm 0.14$ & $-2.36 \pm 0.09$ & 0.221 & 0.647 & rs & rs & 10.6 & $\mathrm{U}, \mathrm{H}$ & $\mathrm{Y}$ & $\mathrm{e}$ \\
\hline HE 0111-1346 & $4687 \pm 102$ & $1.26 \pm 0.30$ & $1.77 \pm 0.16$ & $-2.10 \pm 0.09$ & 0.673 & 0.825 & $\mathrm{~s}$ & $\mathrm{~s}$ & 10.9 & $\mathrm{H}$ & $\mathrm{Y}$ & $b, f$ \\
\hline HE 0151-0341 & $4820 \pm 112$ & $1.15 \pm 0.08$ & $1.72 \pm 0.16$ & $-2.89 \pm 0.08$ & 0.303 & 0.607 & rs & rs & 8.9 & HI & $\mathrm{Y}$ & $\mathrm{f}$ \\
\hline HE 0319-0215 & $4738 \pm 100$ & $0.66 \pm 0.40$ & $2.16 \pm 0.10$ & $-2.90 \pm 0.10$ & 0.540 & 0.780 & rs & rs & 9.0 & HI & $\mathrm{Y}$ & $\mathrm{f}$ \\
\hline HE $0507-1653$ & $5035 \pm 53$ & $2.39 \pm 0.16$ & $1.53 \pm 0.14$ & $-1.35 \pm 0.10$ & 1.038 & 1.073 & $\mathrm{~s}$ & $\mathrm{~s}$ & 9.0 & $\mathrm{H}$ & $\mathrm{Y}$ & $b, f$ \\
\hline HE $1120-2122$ & $4500 \pm 100$ & $0.50 \pm 0.50$ & $1.50 \pm 0.10$ & $-2.00 \pm 0.10$ & 0.331 & 0.470 & rs & rs & 7.3 & $\mathrm{H}$ & $\mathrm{Y}$ & $\mathrm{b}, \mathrm{c}$ \\
\hline HE $1429-0551$ & $4832 \pm 41$ & $1.14 \pm 0.20$ & $2.01 \pm 0.14$ & $-2.70 \pm 0.10$ & 0.714 & 0.777 & rs & $\mathrm{s}$ & 4.1 & $\mathrm{H}$ & $\mathrm{N}$ & $\mathrm{b}, \mathrm{c}$ \\
\hline HE 2144-1832 & $4250 \pm 100$ & $0.50 \pm 0.30$ & $2.00 \pm 0.15$ & $-1.85 \pm 0.10$ & 0.753 & 0.798 & rs & $\mathrm{s}$ & 5.1 & $\mathrm{H}$ & $\mathrm{Y} ?$ & $\mathrm{~b}, \mathrm{c}$ \\
\hline HE $2255-1724$ & $4776 \pm 51$ & $1.64 \pm 0.15$ & $1.84 \pm 0.15$ & $-2.32 \pm 0.09$ & 0.744 & 0.875 & $\mathrm{~s}$ & $\mathrm{~s}$ & 7.4 & $\mathrm{HI}$ & - & - \\
\hline
\end{tabular}

Notes. Columns 2-5 list the atmospheric parameters ( $\xi$ is the microturbulence velocity). The $d_{\mathrm{S}}$ and $d_{\text {rms }}$ distances are described in Sect. 5 . Column 8, labelled "Orig. class.", specifies the classification based on $[\mathrm{La} / \mathrm{Eu}]$, adopting the criterion suggested by Beers \& Christlieb (2005), whereas Col. 9, labelled "New class.", refers to the star assignment adopted in the present paper (see Sect. 5). The $\chi^{2}$ indicator (Eq. (6) in Sect. 7) quantifies the agreement between the AGB model predictions and the measured abundances of eight heavy elements. Column 11, labelled "Spec", refers to the spectrograph used (U, H, and HI correspond to UVES, HERMES, and HIRES, respectively). A question mark in the "Bin" column flags a possible spectroscopic binary. ${ }^{(1)} \mathrm{HD} 221170$ is an $r$-process-enriched star not enriched in carbon.

References. References for the "Bin" column: (a) HERMES unpublished data; (b) Jorissen et al. (2016); (c) Jorissen et al. (in prep.); (d) Preston \& Sneden (2001); (e) McClure \& Woodsworth (1990); (f) Hansen et al. (2016a); (g) Jorissen et al. (2005); (h) Pereira et al. (2019); (i) Hansen et al. (2015).

equilibrium techniques to estimate the atmospheric parameters is challenging. As an initial estimate we derived the photometric temperatures using the relations given by Alonso et al. (1996, 1999) for dwarfs and giants. These calibrations use Johnson UBVRI colours, and the Telescopio Carlos Sanchez (TCS) system for the infrared colours $J-H$ and $J-K$. We performed the necessary transformation to these photometric systems using the transformation relations from Ramírez \& Meléndez (2004) and Alonso et al. (1996, 1999).

With the help of the BACCHUS pipeline (Masseron et al. 2016), which uses interpolated MARCS model atmospheres (Gustafsson et al. 2008) and the 1D local thermodynamical equilibrium (LTE) spectrum-synthesis code TURBOSPECTRUM (Alvarez \& Plez 1998; Plez 2012), we derived the atmospheric parameters for the programme stars. The Brussels Automatic Code for Characterizing High accUracy Spectra (BACCHUS) derives the stellar parameters $\left(T_{\text {eff }},[\mathrm{Fe} / \mathrm{H}], \log g\right.$, microturbulence velocity $\xi$, and rotational velocity). The code includes on-the-fly spectrum synthesis, local continuum normalization, estimation of local $\mathrm{S} / \mathrm{N}$, and automatic line masking. It computes abundances using equivalent widths or spectral synthesis, allowing us to check for excitation and ionization equilibria, thereby constraining $T_{\text {eff }}$ and $\log g$. The microturbulent velocity $\xi$ is calculated by ensuring consistency between Fe abundances derived from lines of various reduced equivalent widths.
We manually selected clean $\mathrm{Fe}$ lines to avoid possible errors due to blends. We performed the spectral fitting of the $\mathrm{C}_{2}$ band at $5165 \AA$ and of the $\mathrm{CN}$ region around $6320 \AA$ using these parameters to get an initial estimate of the $\mathrm{C}$ and $\mathrm{N}$ abundances. We included these values in the BACCHUS initial abundances so as to derive the equivalent widths of $\mathrm{Fe}$ lines by taking care of the possible blends caused by these elements, and we then derived the final parameters. In order to check the quality of the spectral fit with the adopted atmospheric parameters, we used the KASTEEL code, which relies on a spectral fitting method of selected wavelength ranges sensitive to the atmospheric parameters (see Fig.1). It uses the same radiative transfer code and line lists as BACCHUS to minimize systematics. Here we focus on eight wavelength ranges, namely around the $\mathrm{CN}$ bands and Ca II H\&K lines [3860-3985 ̊] , the CH band [3985-4050 А], the $\mathrm{CN}$ blend at $4215 \AA$ and the $\mathrm{Ca} \mathrm{I}$ line at $4226 \AA$ [4210-4240 $\AA$ ], the ${ }^{12} \mathrm{C}^{12} \mathrm{C}$ and ${ }^{12} \mathrm{C}^{13} \mathrm{C}$ bands [4725-4755 $\AA$ ], the region around $\mathrm{H} \beta[4830-4890 \AA]$, the $\mathrm{C}_{2}$ Swan band and the Mg I b triplet [5150-5190 ̊], the [O I] line [6355-6375 $\AA$ ], and the region around $\mathrm{H} \alpha$ including the $\mathrm{Ba}$ II line at $6496.9 \AA$ [6490-6600 $\mathrm{A}]$. A few Fe I and Fe II lines in the spectral regions 5200-5400 $\AA$ and 5800-5900 , which are comparatively free from molecular blending, were visually tested to validate the atmospheric parameters, especially $\log g$ and metallicity. 


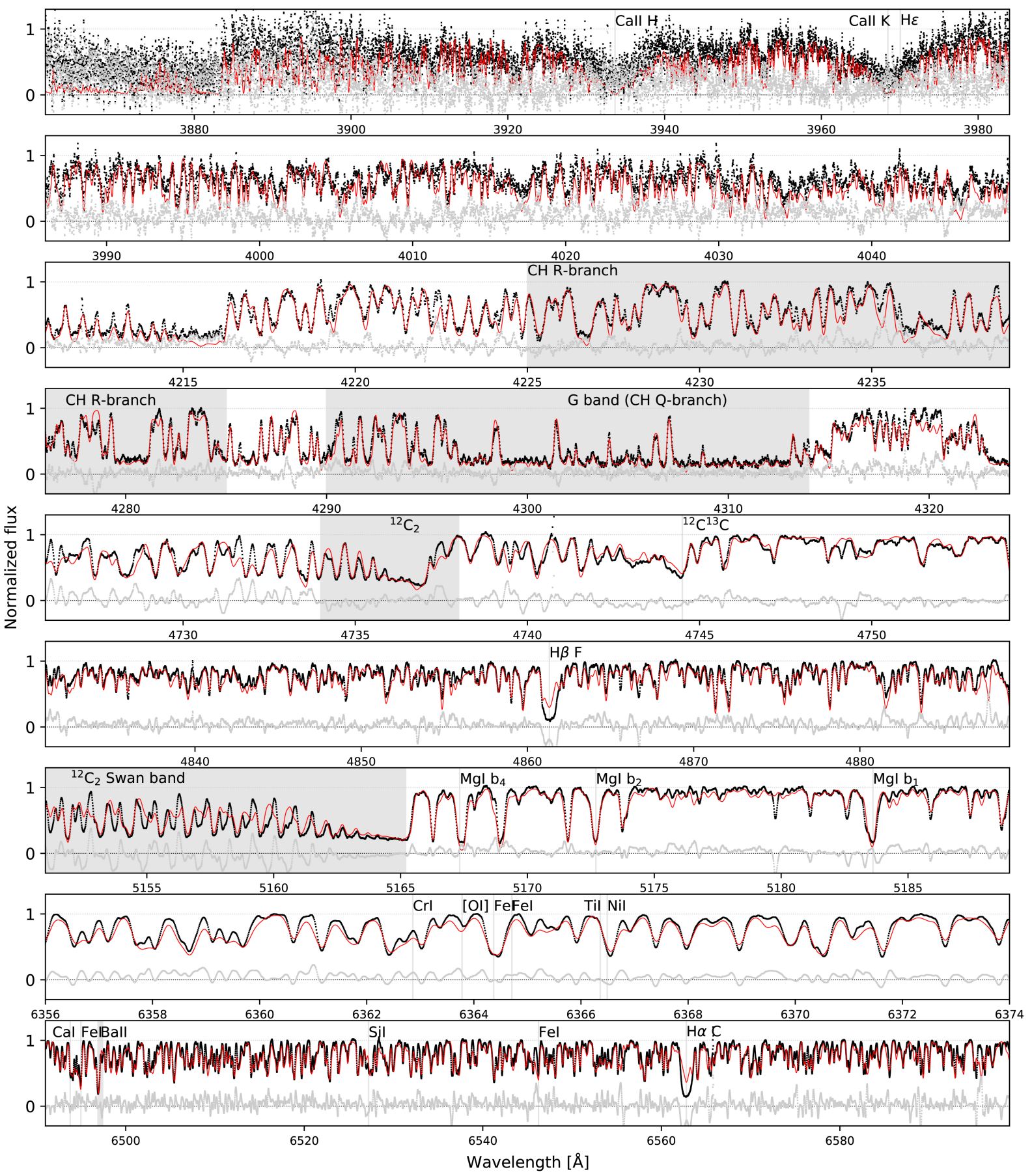

Fig. 1. Spectral fitting of HE 1120-2122 using KASTEEL. Different spectral regions used to fine-tune the atmospheric parameters are illustrated. The black dots represent the normalized observed flux, the red line corresponds to the synthetic fluxes, and the grey dots are the residuals. The shaded areas correspond to molecular bands, as labelled.

\section{Abundance determination}

Abundances were derived by comparing observed and synthetic spectra generated with the TURBOSPECTRUM radiative transfer code (Alvarez \& Plez 1998) using MARCS model atmospheres. We used carbon-enriched MARCS models computed by Masseron whenever available. We adopted the solar abundances from Asplund et al. (2009). The line lists assembled in the framework of the Gaia-ESO survey were used (Heiter et al. 2015, 2021). The individual atomic lines, including hyperfine (HF) splitting when available, used for the abundance determina- tion are listed in Table A.1. We derived the abundances under the assumption of LTE, but non-LTE corrections are applied whenever available. In the following we comment on individual elemental abundances.

\section{1. $C, N$, and $O$}

Oxygen abundances were derived first from the [OI] line at $6300.303 \AA$ whenever it could be satisfactorily reproduced. We also used the O I resonance triplet at $7774 \AA$. This feature yields 
higher abundances (by 0.2 to 0.3 dex) in all the objects compared to the abundance derived from the [OI] line. This difference is usually due to NLTE effects strongly affecting the resonance line as described by Asplund et al. (2005) and Amarsi et al. (2016). Hence, we used the $\mathrm{O}$ abundance derived from the [O I] line, or if not available, from the NLTE-corrected abundance from the O I resonance triplet. In Table B.2, we present the oxygen abundances only for the objects for which we could use either the $6300.303 \AA$ or the $7774 \AA$ line. For a few cool stars for which we could detect neither the $6300.303 \AA$ [O I] line nor the triplet at $7774 \AA$, we used the $\alpha$-element abundances scaled to the corresponding stellar metallicity.

$\mathrm{C}$ and $\mathrm{N}$ abundances were derived for all programme stars. The carbon abundance was derived mainly from the $\mathrm{C}_{2}$ bands at 4737,5165 , and $5635 \AA$, avoiding the band heads since they are often saturated and their depth is difficult to reproduce, and also from the $\mathrm{CH} G$ band at $4310 \AA$ (Fig. 2). We were able to get abundances consistent within 0.2 dex from all these bands.

The nitrogen abundance for the programme stars observed with the UVES spectrograph was derived from the CN bands at 3883,4215 , and $6323 \AA$. For objects with spectra acquired with the HERMES spectrograph, we also used $\mathrm{CN}$ lines above $7500 \AA$. $\mathrm{N}$ abundances derived from these different bands are found to be consistent within 0.15 dex.

The ${ }^{12} \mathrm{C} /{ }^{13} \mathrm{C}$ ratio was derived using ${ }^{12} \mathrm{CN}$ features at 8003.553 and $8003.910 \AA$ and ${ }^{13} \mathrm{CN}$ features at 8004.554 , 8004.728 and $8004.781 \AA$. We also used the ${ }^{13} \mathrm{CN}$ features at 8010.458 and $8016.429 \AA$, the ${ }^{12} \mathrm{C}^{12} \mathrm{C}$ feature at $4737 \AA$, and the ${ }^{12} \mathrm{C}^{13} \mathrm{C}$ feature at $4744 \AA$ as a consistency check. The ${ }^{12} \mathrm{C} /{ }^{13} \mathrm{C}$ values from these various bands are found to agree within \pm 5 .

\subsection{Light elements}

The abundances of $\mathrm{Na}, \mathrm{Mg}, \mathrm{Ca}, \mathrm{Ti}, \mathrm{Sc}, \mathrm{Cr}, \mathrm{Mn}, \mathrm{Co}, \mathrm{Cu}$, and $\mathrm{Zn}$ were derived (Table B.1) using the lines listed in Table A.1.

\subsection{1. $\mathrm{Na}$}

The $\mathrm{Na}$ abundance was derived using mainly two Na I lines at 5682.633 and $5688.205 \AA$. We also used the NaI lines at 6154.226 and $6160.747 \AA$ whenever available. The lines used to derive the $\mathrm{Na}$ abundance are weak and are known to be free from NLTE effects.

\subsection{2. $\mathrm{Mg}, \mathrm{Ca}, \mathrm{Sc}, \mathrm{Ti}$}

We could measure the $\mathrm{Mg}$ abundance only in a few stars using the $\mathrm{Mg}$ lines at 4571.096, 4702.991, 5528.405, and 5711.088. . Although many lines of the elements $\mathrm{Ca}, \mathrm{Sc}$, and Ti could be identified, only those listed in Table A.1 were used to derive abundances. We note that in all our programme stars these $\alpha$ elements are enhanced with an average $[\alpha / \mathrm{Fe}]$ of 0.5 dex.

\section{3. $\mathrm{Cr}, \mathrm{Mn}, \mathrm{Cu}, \mathrm{Ni}, \mathrm{Zn}$}

While $\mathrm{Cr}, \mathrm{Mn}, \mathrm{Cu}$, and $\mathrm{Ni}$ have either subsolar or solar abundances in all our programme stars, we found that $\mathrm{Zn}$ is slightly enriched. HD 55496 shows a rather high enrichment of $\mathrm{Zn}$ with $[\mathrm{Zn} / \mathrm{Fe}]=0.74$. This is reminiscent of the depletion pattern observed in some RV Tauri stars and related objects (Reyniers \& Van Winckel 2007, and references therein), i.e. of an anti-correlation between condensation temperatures in grains
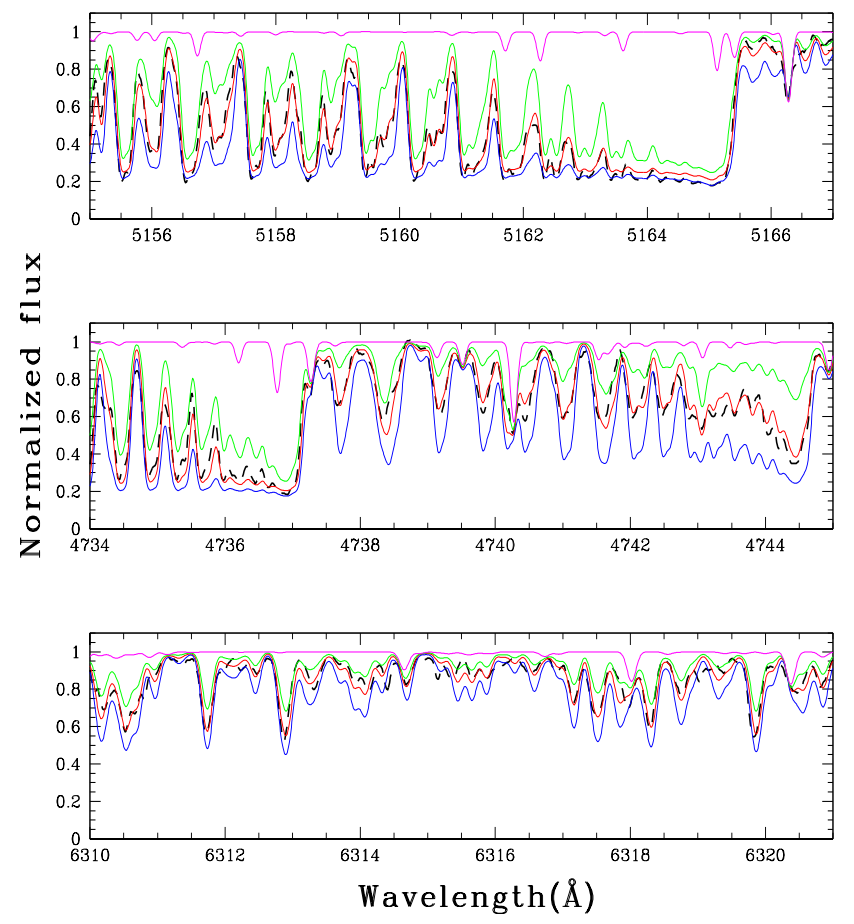

Fig. 2. Spectral fits of the $\mathrm{C}_{2}$ and $\mathrm{CN}$ regions in HD 187861. Upper panel: $\mathrm{C}_{2}$ band around $5165 \AA$ where the red curve represents the synthesis corresponding to the abundance $\log \epsilon_{\mathrm{C}}=8.3$, with $\pm 0.3 \mathrm{dex}$ on either side represented by the blue and green curves. The black dashed line corresponds to the observed spectrum and the magenta line to a spectral synthesis without carbon. Middle panel: spectral synthesis of the ${ }^{12} \mathrm{C}^{12} \mathrm{C}$ feature at $4737 \AA$ and the ${ }^{12} \mathrm{C}^{13} \mathrm{C}$ feature at $4744 \AA$ with the adopted $\mathrm{C}$ abundance and derived isotopic ratio of 16 . The spectral synthesis of the $\mathrm{CN}$ band around $6315 \AA$ is shown in the bottom panel with the adopted abundances of 8.3 and 7.85 for $\mathrm{C}$ and $\mathrm{N}$, respectively.

and elemental abundances. Zinc is a relatively volatile element given its low condensation temperature $\left(T_{\text {cond, } 50 \%}=726 \mathrm{~K}\right.$, Lodders 2003). We checked among all our stars that there is no obvious anti-correlation between condensation temperatures and elemental abundances. If there was any (as super-solar Zn abundances would tend to indicate), it has largely been erased by a subsequent heavy-element enrichment.

\subsection{Light s-process elements: $\mathrm{Sr}, \mathrm{Y}$, and $\mathrm{Zr}$}

Several clean (i.e. unblended) lines are available to derive the abundances of light $s$-elements.

The $\mathrm{Sr}$ abundance was derived using the $\mathrm{SrI}$ lines at 4607.327 and $7070.070 \AA$ or the SrII lines at 4077.707 and 4215.519 A. These Sr lines (listed in Table A.1) are affected by NLTE effects; we now detail star by star the NLTE corrections that were adopted from Mashonkina et al. (2008), Andrievsky et al. (2011), and Bergemann et al. (2012).

CS 22887-048, CS 22942-019, HE 0507-1653, and HE 2144-1832. We used only the SrII line at 4077.707 $\AA$. NLTE corrections corresponding to the stellar parameters of these objects are small, however, ranging from 0.0 to $-0.05 \mathrm{dex}$ (Bergemann et al. 2012).

HD 209621, HD 224959, and HE 1120-2122. The strontium abundance is derived using the Sr I line at $4607.327 \AA$, known to show very large $(\approx 0.3$ to $0.5 \mathrm{dex})$ NLTE corrections at low metallicities $(-3.9 \leq[\mathrm{Fe} / \mathrm{H}] \leq-1.2)$, as listed in Table 3 of 
Bergemann et al. (2012). These authors estimated a NLTE correction of $0.47 \mathrm{dex}$ for $\mathrm{HD} 122563\left(T_{\mathrm{eff}}=4600 \mathrm{~K}, \log g=1.6\right.$, $[\mathrm{Fe} / \mathrm{H}]=-2.5)$. We adopted this value to correct the Sr abundance in these three stars.

HD 5223 and HD 145777. Their Sr abundance was derived using the Sr II line at $4215.52 \AA$ which is known to have a small NLTE correction $(\approx-0.05 \mathrm{dex})$ in metal-poor stars (Mashonkina et al. 2008; Andrievsky et al. 2011).

HD 26 and HD 55496. We used the Sr I line at 7070.070 for which NLTE investigations are not available in the literature.

The $\mathrm{Y}$ abundances for the programme stars were derived from the Y II lines listed in Table A.1. No NLTE correction is available for these lines. The $\mathrm{Zr}$ abundance was derived mainly using the $\mathrm{Zr}$ II lines at 5112.270 and $5350.350 \AA$. We could not find any useful $\mathrm{Zr}$ I lines; in particular, we could not use the lines at 7819.374 and $7849.365 \AA$ with laboratory $\log g f$ values as in Karinkuzhi et al. (2018).

\subsection{Heavy s-process elements: $\mathrm{Ba}, \mathrm{La}, \mathrm{Ce}, \mathrm{Pr}, \mathrm{Nd}$}

Since most of the $\mathrm{Ba}$ lines are very strong in our programme stars, the $\mathrm{Ba}$ abundance is derived mainly from the spectral synthesis of the weak Ba II line at $4524.924 \AA$. For a few objects, we could also use the line at $5853.673 \AA$. In the case of CS 22887-048 we used the Ba II line at $4166.000 \AA$. Ba lines are strongly affected by HF splitting. HF splitting data is not available for the $4524.924 \AA$ line, but it was taken into account for the Ba II line at $5853.673 \AA$. NLTE corrections for the $\mathrm{Ba}$ line at $5853.673 \AA$ is negligible for dwarfs and giants, as concluded by Mashonkina et al. (1999), Mashonkina \& Gehren (2000), and Andrievsky et al. (2009) for the metallicity and temperature ranges of our objects. For CS 30322-023, HD 55496, HD 221170, and HE 2255-1724 we also used the Ba II line at $5853.673 \AA$ to derive the Ba abundance. NLTE corrections corresponding to the parameter range of these four objects are from Andrievsky et al. (2009) and range between 0.0 and 0.07. We present LTE and NLTE Ba abundances separately in Table B.2 for these four objects, where $\mathrm{Ba}_{\mathrm{LTE}}$ indicates the $\mathrm{Ba}$ abundance derived using the $\mathrm{Ba}$ II line at $4524.924 \AA$ and $\mathrm{Ba}_{\mathrm{NLTE}}$ indicates that from $5853.673 \AA$ line after considering the NLTE correction.

The La abundance is derived mainly using the lines for which HF splitting is available. Although Nd II lines are available throughout the spectrum, we used mainly Nd II lines in the range 5200-5400 $\AA$ since they are relatively free from molecular blends. The Ce II and Pr II abundances were also derived in all the programme stars. Spectral fitting of a few lines is shown in Fig. 3.

\section{6. r-process-dominated elements: Sm, Eu, Dy, Gd, Er, Hf, Os, Ir}

The Solar System enrichment in Sm, Eu, Dy, Gd, Er, Os, and Ir is believed to originate from the $r$-process nucleosynthesis by more than $80 \%$ (Goriely 1999). The solar Hf is expected to be produced by the $s$ - and $r$-process in approximately the same amount. Globally, these elements play a key role in tracing large neutron irradiations responsible for their production; they are thus often referred to as $r$-process elements.

Most of the Sm II lines used to derive the Sm abundance are blended with bands from carbon-bearing molecules. These were carefully reproduced before deriving Sm abundances.

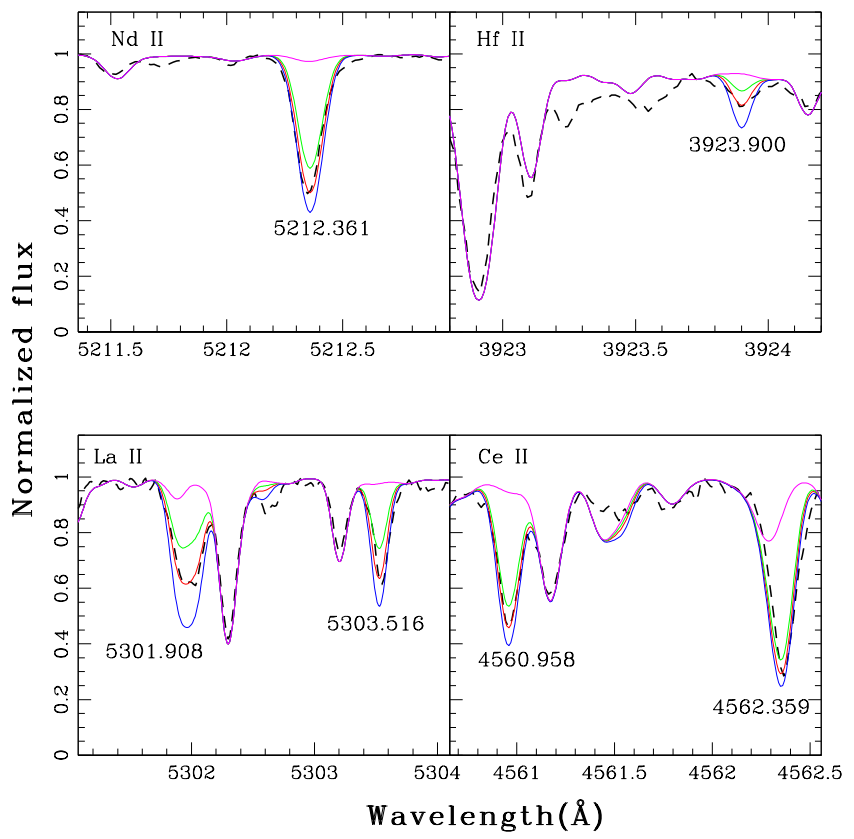

Fig. 3. Spectral fitting of the La II and Ce II lines is shown for HD 198269 in the lower panel. Spectral synthesis of Nd II and Hf II is shown for HD 224959 in the upper panel. Red curves correspond to spectral syntheses with the adopted abundances for these elements, as listed in Table B.2. The blue and green curves correspond to syntheses with abundances deviating by \pm 0.3 dex from the adopted abundance. The black dashed line represents the observed spectrum. The magenta line corresponds to the synthesis with a null abundance for the corresponding element.

The Eu abundance was derived using four Eu II lines with $\mathrm{HF}$ splitting available. As for the $\mathrm{Sm}$ lines, the $\mathrm{Eu}$ lines are blended with the $\mathrm{CH}$ and $\mathrm{CN}$ lines. The Eu abundance, when derived using two blue lines at 4129.720 and $4205.065 \AA$, is always lower by approximately 0.1 to 0.2 dex compared to the abundance derived from the two red lines. Mashonkina et al. (2008) calculated the NLTE corrections for the Eu II line at $4129.720 \AA$ in the two metal-poor stars HD 122563 $\left(T_{\text {eff }}=4600 \mathrm{~K}, \log g=1.50,[\mathrm{Fe} / \mathrm{H}]=-2.53\right)$, and HD 84937 $\left(T_{\mathrm{eff}}=6365 \mathrm{~K}, \log g=4.00,[\mathrm{Fe} / \mathrm{H}]=-2.15\right)$ and found corrections of respectively 0.16 and 0.12 dex. Since our programme star parameters are close to those of these two objects, the differences in $\mathrm{Eu}$ abundances when measured using the 4129.720 A line instead can be explained with these NLTE corrections. We could use the $4129.720 \AA$ Eu line in four objects, namely CS 22887-048, CS 22891-171, CS 22942-019, and HD 221170. We present LTE and NLTE Eu abundances separately in Table B. 2 for these objects. Hence, $\mathrm{Eu}_{\mathrm{NLTE}}$ denotes the abundance derived using the $4129.720 \AA$ line after applying the corresponding NLTE correction, while $\mathrm{Eu}_{\mathrm{LTE}}$ denotes the average Eu abundance derived using all Eu lines available excluding the $4129.720 \AA$ line. For HD 196944 we used the two Eu II lines at 3907.10 and $4205.065 \AA$ to derive the $\mathrm{Eu}$ abundance. Mashonkina \& Christlieb (2014) listed the NLTE corrections of 0.28 and 0.15 dex respectively for these two lines, corresponding to the parameters $T_{\text {eff }}=4750 \mathrm{~K}, \log g=1.00,[\mathrm{Fe} / \mathrm{H}]=-3.00$, which are closest to those of HD 196944. The Eu $\mathrm{ELTE}_{\text {NLT }}$ abundance presented in Table B.2 for HD 196944 is the average of the abundances derived from these two lines after applying NLTE corrections. For HE0151-0341 the Eu abundance is derived using Eu II line at $4205.065 \AA$. We applied NLTE 


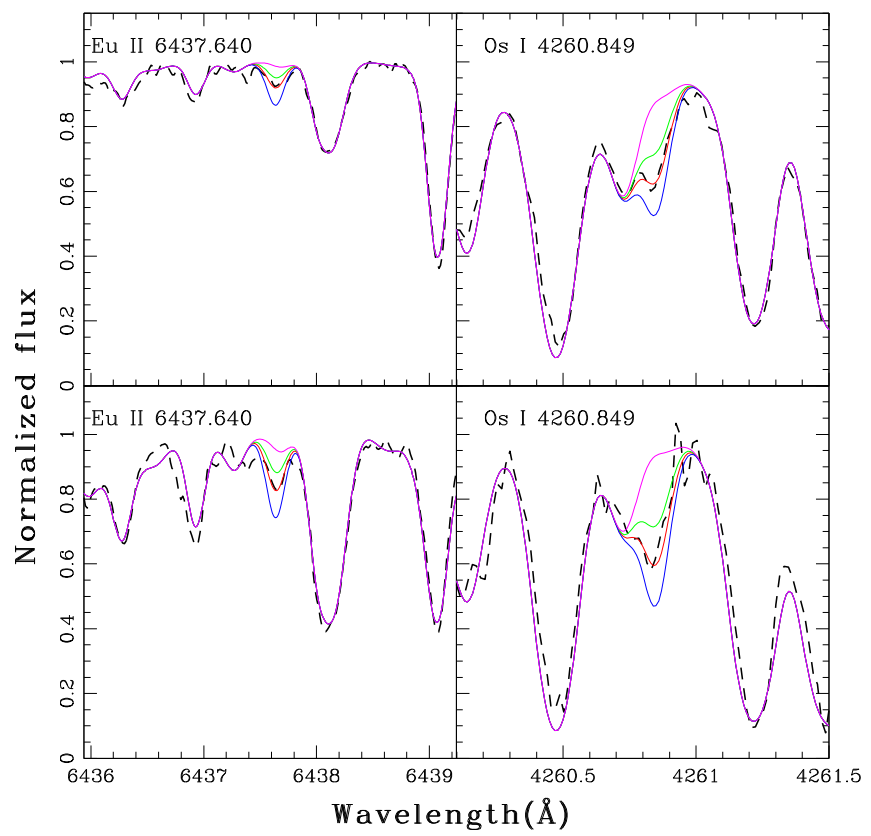

Fig. 4. Spectral fits of the Eu II line at $6437.640 \AA$ and of the Os line at $4260.849 \AA$ are shown for HD 5223 in the lower panel and for HD 198269 in the upper panel. The red curve corresponds to syntheses with the adopted abundances ( -0.3 dex for Eu and 0.4 dex for Os in HD 5223, and -0.91 dex and -0.15 dex in HD 198269). The blue, green, magenta, and black curves are as in Fig. 3.

correction of $0.15 \mathrm{dex}$ corresponding to $T_{\mathrm{eff}}=4750 \mathrm{~K}, \log g=$ 1.00, $[\mathrm{Fe} / \mathrm{H}]=-3.00$ from Mashonkina \& Christlieb (2014). For the $6645.130 \AA \mathrm{Eu}$ II line these corrections are very small $(\approx 0.06$ to 0.08 dex in main-sequence and turn-off stars with metallicities higher than -3.0 (Mashonkina \& Gehren 2000; Mashonkina \& Christlieb 2014). NLTE correction is not available for Eu II line at $6437.640 \AA$. The final Eu abundance given in Table B. 2 for all other targets is the average of the abundances from all the available Eu lines.

Abundances of $\mathrm{Gd}$ and Os were measured using a single Gd II line at $4251.731 \AA$ and a Os I line at $4260.849 \AA$, respectively. We present the spectral fitting of the Os I line for HD 5223 and HD 198269 in Fig. 4. Since most of the good Er lines are located at the blue end of the spectrum, we could derive Er abundances in only a few stars. We used the Er II line at 3906.311 for most of the stars. The Ir abundance was measured only in HE 1120-2122, using the Ir I line at 3992.121 A.

\subsection{The third peak s-process element $\mathrm{Pb}$}

The $\mathrm{Pb}$ abundance was derived using the $\mathrm{Pb}$ I line at $4057.807 \AA$, taking into account HF splitting. NLTE corrections for this $\mathrm{Pb}$ line are listed in Mashonkina et al. (2012) for a range of atmospheric parameters. The NLTE corrections for our objects vary from 0.3 to $0.5 \mathrm{dex}$, and the adopted correction corresponds to the model (from Table 1 of Mashonkina et al. 2012) closest to the atmospheric parameters of the considered star. As for Eu, we list both LTE and NLTE Pb abundances in Table B.2.

\subsection{Uncertainties on the abundances}

We estimated the uncertainties on all the elemental abundances $\log \epsilon$ following Eq. (2) from Johnson (2002):

$$
\begin{aligned}
\sigma_{\text {tot }}^{2}= & \sigma_{\text {ran }}^{2}+\left(\frac{\partial \log \epsilon}{\partial T}\right)^{2} \sigma_{T}^{2}+\left(\frac{\partial \log \epsilon}{\partial \log g}\right)^{2} \sigma_{\log g}^{2} \\
& +\left(\frac{\partial \log \epsilon}{\partial \xi}\right)^{2} \sigma_{\xi}^{2}+\left(\frac{\partial \log \epsilon}{\partial[\mathrm{Fe} / \mathrm{H}]}\right)^{2} \sigma_{[\mathrm{Fe} / \mathrm{H}]}^{2} \\
& +2\left[\left(\frac{\partial \log \epsilon}{\partial T}\right)\left(\frac{\partial \log \epsilon}{\partial \log g}\right) \sigma_{T \log g}+\left(\frac{\partial \log \epsilon}{\partial \xi}\right)\left(\frac{\partial \log \epsilon}{\partial \log g}\right) \sigma_{\log g \xi}\right. \\
& \left.+\left(\frac{\partial \log \epsilon}{\partial \xi}\right)\left(\frac{\partial \log \epsilon}{\partial T}\right) \sigma_{\xi T}\right]
\end{aligned}
$$

In Eq. (1) the values $\sigma_{T}, \sigma_{\log g}$, and $\sigma_{\xi}$ are the typical uncertainties on the atmospheric parameters, which are estimated as $\sigma_{T}=80 \mathrm{~K}, \sigma_{\log g}=0.3 \mathrm{dex}, \sigma_{\xi}=0.1 \mathrm{~km} \mathrm{~s}^{-1}$, and $\sigma_{[\mathrm{Fe} / \mathrm{H}]}=0.15 \mathrm{dex}$. The partial derivatives appearing in Eq. (1) were evaluated in the specific cases of HD 196944 and HD 198269, varying the atmospheric parameters $T_{\text {eff }}, \log g$, microturbulence $\xi$, and $[\mathrm{Fe} / \mathrm{H}]$ by $100 \mathrm{~K}, 0.5,0.5 \mathrm{~km} \mathrm{~s}^{-1}$, and 0.5 dex, respectively. The resulting changes in the abundances are presented in Table 2. The covariances $\sigma_{T \log g}, \sigma_{\log g \xi}$, and $\sigma_{\xi T}$ were derived using the same method as given in Johnson (2002). In order to calculate $\sigma_{T \log g}$, we varied the temperature while fixing metallicity and microturbulence, and determined the $\log g$ value required to ensure the ionization balance. Then using Eq. (3) in Johnson (2002), we derived the covariance $\sigma_{T \log g}$ and found a value of -0.5 . In a similar way, we found $\sigma_{\log g \xi}=0.02$ and $\sigma_{\xi T}=3$.

Finally, the random error $\sigma_{\text {ran }}$ is the line-to-line scatter. For most of the elements, we could use more than four lines to derive the abundances. In those cases we adopted $\sigma_{\text {ran }}=\sigma_{1} / N^{1 / 2}$, where $\sigma_{1}$ is the standard deviation of the abundances derived from all the $N$ lines of the considered element. It involves uncertainties caused by factors like line blending, continuum normalization, and oscillator strength. However, for elements like $\mathrm{Zr}$, $\mathrm{Eu}, \mathrm{Dy}, \mathrm{Er}$, and Hf, only two or three lines could be used, so that the above method had to be adapted. For instance, in the case of $\mathrm{Zr}$ (but we used the same procedure for $\mathrm{Eu}, \mathrm{Dy}, \mathrm{Er}$, and $\mathrm{Hf}$ ), we first calculate $\sigma_{\mathrm{Zr} \text {,avg }}$, which is the average standard deviation on $\mathrm{Zr}$ abundances on all the stars in our sample. Then we define $\sigma_{\mathrm{Zr} \text {,ran }}$ as $\sigma_{\mathrm{Zr} \text {,avg }} / N^{1 / 2}$, where $N$ is the number of considered $\mathrm{Zr}$ lines in a star with $N<4$. For elements $\mathrm{Sr}, \mathrm{Ba}, \mathrm{Gd}$, Os, and $\mathrm{Pb}$ we used only one line to measure the abundance in all the programme stars. Hence, we adopted $\sigma_{\text {ran }}=0.1 \mathrm{dex}$ for these elements, which is the minimum abundance difference yielding a clear difference in the synthetic spectra. Finally, the error on $[\mathrm{X} / \mathrm{Fe}]$ was derived from

$\sigma_{[\mathrm{X} / \mathrm{Fe}]}^{2}=\sigma_{\mathrm{X}}^{2}+\sigma_{\mathrm{Fe}}^{2}-2 \sigma_{\mathrm{X}, \mathrm{Fe}}$,

where $\sigma_{\mathrm{X}, \mathrm{Fe}}$ was calculated using Eq. (6) from Johnson (2002).

\section{Re-assignment of the programme stars}

CEMP stars are defined as low-metallicity stars (typically $[\mathrm{Fe} / \mathrm{H}] \leq-1$ ) with typically $[\mathrm{C} / \mathrm{Fe}]>+1$ (Beers \& Christlieb 2005). However, different carbon abundance thresholds are used in the literature to identify stars as CEMPs. Aoki et al. (2007) use the following criteria: $[\mathrm{C} / \mathrm{Fe}]>0.7$ for stars with $\log \left(L / L_{\odot}\right) \leq 2.3$ and $[\mathrm{C} / \mathrm{Fe}] \geq 3-\log \left(L / L_{\odot}\right)$ for stars with $\log \left(L / L_{\odot}\right)>2.3$. Masseron et al. (2010) use instead a threshold of $[\mathrm{C} / \mathrm{Fe}]>0.9$ to define CEMP stars. However, a classification based on the $\mathrm{C}$ abundance alone might be misleading when considering low-metallicitiy nucleosynthesis. Some stars on the red giant branch (RGB) experience $\mathrm{CN}$ processing which will reduce the surface $\mathrm{C}$ abundance. The $[\mathrm{C} / \mathrm{Fe}]$ 
Table 2. Sensitivity of the abundances $\left(\Delta \log \epsilon_{\mathrm{X}}\right)$ with variations in the atmospheric parameters (considering the atmospheric parameters of HD 198269).

\begin{tabular}{|c|c|c|c|c|}
\hline \multirow[b]{2}{*}{ Element } & \multicolumn{4}{|c|}{$\Delta \log \epsilon_{\mathrm{X}}$} \\
\hline & $\begin{array}{r}\Delta T_{\text {eff }} \\
(+100 \mathrm{~K})\end{array}$ & $\begin{array}{r}\Delta \log g \\
(+0.5)\end{array}$ & $\begin{array}{r}\Delta \xi_{\mathrm{t}} \\
(+0.5 \\
\left.\mathrm{km} \mathrm{s}^{-1}\right)\end{array}$ & $\begin{array}{r}\Delta[\mathrm{Fe} / \mathrm{H}] \\
(+0.5 \\
\operatorname{dex}) \\
\end{array}$ \\
\hline $\mathrm{C}$ & 0.04 & 0.06 & -0.03 & -0.05 \\
\hline $\mathrm{N}$ & 0.21 & 0.19 & 0.55 & 0.14 \\
\hline $\mathrm{O}$ & 0.00 & 0.20 & -0.10 & -0.10 \\
\hline $\mathrm{Na}$ & 0.08 & 0.05 & 0.13 & 0.23 \\
\hline $\mathrm{Mg}$ & 0.10 & 0.00 & 0.00 & 0.00 \\
\hline $\mathrm{Fe}$ & 0.12 & 0.07 & -0.12 & 0.04 \\
\hline $\mathrm{Sr}$ & 0.05 & -0.05 & -0.20 & 0.00 \\
\hline $\mathrm{Y}$ & 0.01 & 0.16 & 0.04 & -0.08 \\
\hline $\mathrm{Zr}$ & 0.00 & 0.07 & -0.10 & -0.03 \\
\hline $\mathrm{Ba}$ & 0.00 & 0.10 & 0.05 & 0.00 \\
\hline $\mathrm{La}$ & 0.04 & 0.17 & -0.08 & -0.09 \\
\hline $\mathrm{Ce}$ & 0.00 & 0.14 & -0.12 & -0.07 \\
\hline $\operatorname{Pr}$ & 0.03 & 0.14 & -0.06 & -0.10 \\
\hline $\mathrm{Nd}$ & 0.01 & 0.10 & -0.17 & -0.13 \\
\hline $\mathrm{Sm}$ & -0.01 & 0.08 & -0.19 & -0.13 \\
\hline $\mathrm{Eu}$ & -0.03 & 0.13 & -0.05 & -0.18 \\
\hline $\mathrm{Gd}$ & 0.00 & 0.20 & -0.15 & -0.30 \\
\hline Dy & 0.00 & 0.10 & 0.03 & 0.00 \\
\hline $\mathrm{Er}$ & 0.00 & 0.10 & -0.25 & -0.05 \\
\hline $\mathrm{Hf}$ & 0.05 & 0.15 & -0.15 & -0.18 \\
\hline Os & 0.05 & 0.05 & 0.05 & 0.05 \\
\hline $\mathrm{Pb}$ & 0.30 & 0.00 & 0.20 & 0.30 \\
\hline
\end{tabular}

Notes. The lines of a few elements could not be detected in HD 198269; we thus used HD 196944 (slightly higher in temperature, but with otherwise similar parameters) to estimate the abundance sensitivity to parameter changes.

limits set by Aoki et al. (2007) allow more evolved stars to be considered as participating in similar nucleosynthesis processes. In the same vein, among our 25 objects, 3 have $[\mathrm{C} / \mathrm{Fe}]<0.5$. HD 206983 and HD 55496 have low $\mathrm{C}$ abundances $([\mathrm{C} / \mathrm{Fe}]=0.42$ and 0.07 respectively), but definite $s$-process enrichments $([\mathrm{s} / \mathrm{Fe}]=0.59$ and 0.87 , respectively). HD 221170 is an $r$-process-enriched star without carbon enrichment. This confirms that a classification involving enrichments in $s$ - and $r$-process elements, as well as carbon enrichment (even modest levels) is needed to properly classify the full family of metalpoor objects. Currently the abundances of two additional chemical elements are used, namely barium (proxy for $s$-process) and europium (proxy for $r$-process). Here again, the definition of CEMP subgroups vary among authors (Beers \& Christlieb 2005; Jonsell et al. 2006; Masseron et al. 2010; Hollek et al. 2015; Abate et al. 2016). Beers \& Christlieb (2005) propose classifying as CEMP-rs the stars with $0<[\mathrm{Ba} / \mathrm{Eu}]<0.5$, with no restrictions on $[\mathrm{Ba} / \mathrm{Fe}]$ or $[\mathrm{Eu} / \mathrm{Fe}]$. Jonsell et al. (2006) modified the Beers \& Christlieb (2005) classification for CEMP-rs stars by imposing $[\mathrm{Ba} / \mathrm{Fe}]>1.0$ and $[\mathrm{Eu} / \mathrm{Fe}]>1.0$. Masseron et al. (2010) followed Jonsell et al. (2006) for CEMP-s and -rs stars, but adopted the definition of CEMP-rI and rII stars from Beers \& Christlieb (2005).

With the derived abundances becoming more accurate, stars with definite (albeit low) levels of ( $s$ - or $r$-) heavy element enrichments are detected. A limit on $[\mathrm{Ba} / \mathrm{Fe}]$ or $[\mathrm{Eu} / \mathrm{Fe}]$ might turn rapidly obsolete, while $[\mathrm{Ba} / \mathrm{Eu}]$ is more robust. This is why

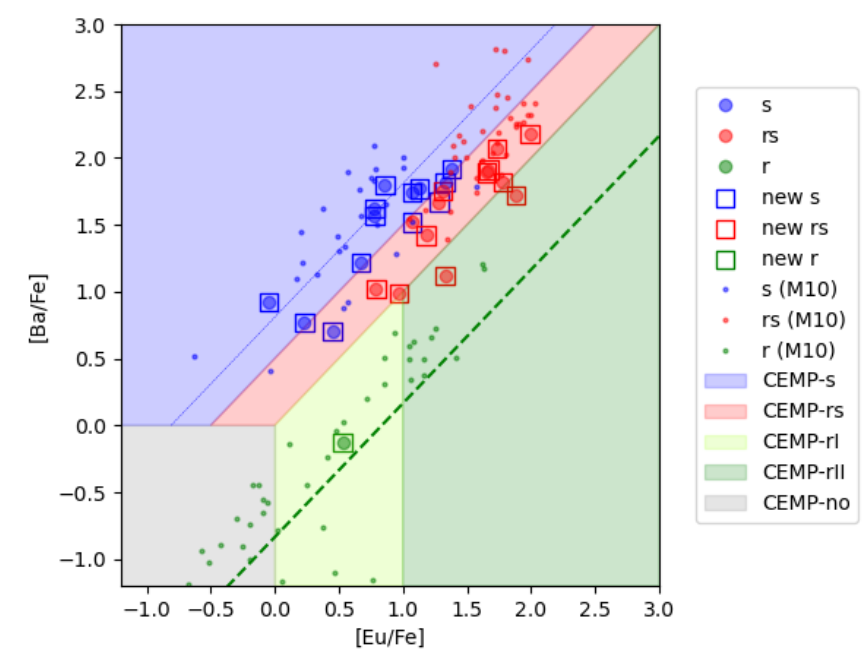

Fig. 5. $[\mathrm{Ba} / \mathrm{Fe}]$ as a function of $[\mathrm{Eu} / \mathrm{Fe}]$. CEMP-s stars, CEMP-rs stars, and an $r$-process enriched star studied in this paper are represented by blue, red, and green filled circles when adopting the original classification (Table 1, Orig. class.) and by large squares with the same colour-coding when adopting our new classification (Col. 9 of Table 1 and Sect. 5). The small dots represent objects compiled from the literature in Masseron et al. (2010). The dashed green line corresponds to abundance-ratio scaling with a pure solar $r$-process (Goriely 1999), whereas the continuous blue line corresponds to $s$-process nucleosynthesis abundance ratio scaling with the predictions for a $1.5 M_{\odot}$ star of $[\mathrm{Fe} / \mathrm{H}]=-1$ (5th pulse).

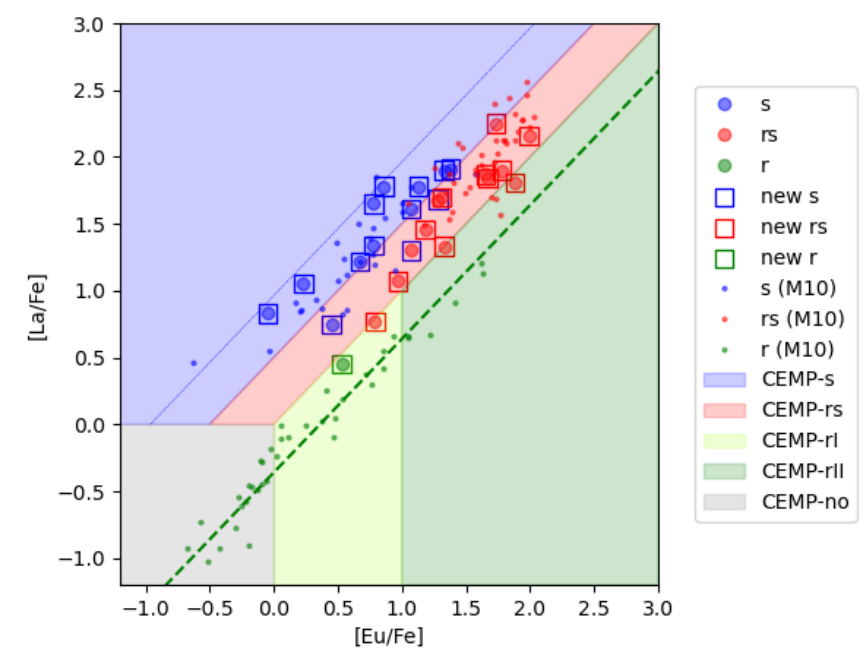

Fig. 6. Same as in Fig. 5, but for $[\mathrm{La} / \mathrm{Fe}]$ as a function of $[\mathrm{Eu} / \mathrm{Fe}]$.

we adopted here the original classification criteria proposed by Beers \& Christlieb (2005), but replace barium by lanthanum, since La abundances are often considered to be more reliable than $\mathrm{Ba}$ abundances because they are often obtained from more numerous, less saturated lines. To summarize, we applied the following classification criteria in Col. 8 of Table 1:

- CEMP-s: $[\mathrm{La} / \mathrm{Eu}]>0.5$;

- CEMP-rs: $0.0<[\mathrm{La} / \mathrm{Eu}]<0.5$;

- CEMP-rI: $[\mathrm{La} / \mathrm{Eu}]<0,0<[\mathrm{Eu} / \mathrm{Fe}]<+1$;

- CEMP-rII: $[\mathrm{La} / \mathrm{Eu}]<0,[\mathrm{Eu} / \mathrm{Fe}]>+1$.

However, these classifications are not without problems. HD 196944 and HD 187861 have [La/Eu] very close to zero and are classified as CEMP-rs (instead of CEMP-r). CS 22891-171, with $[\mathrm{La} / \mathrm{Eu}]=0.55$, has been classified as CEMP-rs (instead 


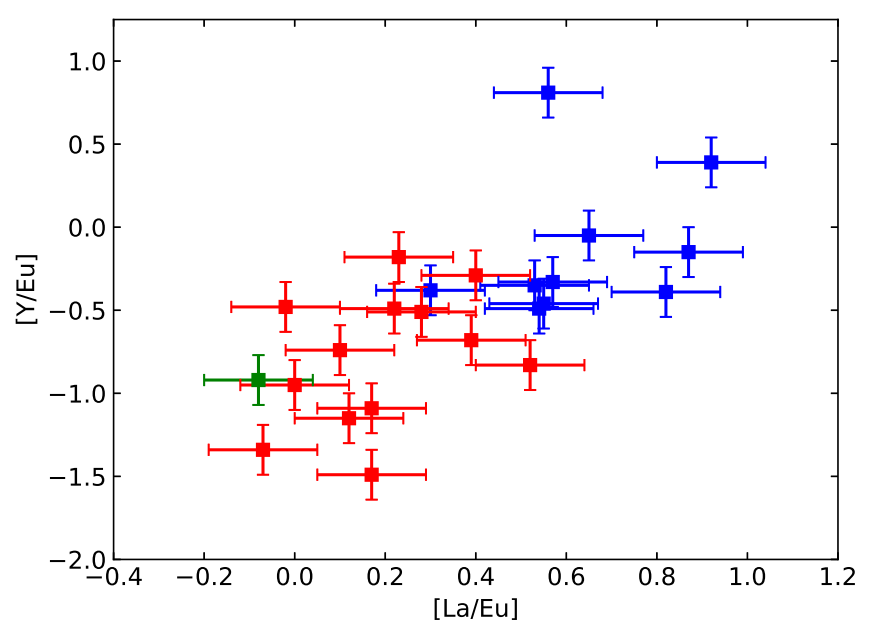

Fig. 7. $[\mathrm{Y} / \mathrm{Eu}]$ as a function of $[\mathrm{La} / \mathrm{Eu}]$ for CEMP-s stars (blue squares), CEMP-rs stars (red squares), and an $r$-process-enriched star (green square), according to Col. 8 of Table 1 .

of CEMP-s), as in Masseron et al. (2010). HD 206983 and HD 55496 have been classified as CEMP-s, as in Pereira et al. (2011, 2019).

Finally, Figs. 5 and 6 present the usual $s$-process diagnostics $([\mathrm{Ba} / \mathrm{Fe}]$ and $[\mathrm{La} / \mathrm{Fe}])$ as a function of an $r$-process diagnostic $([\mathrm{Eu} / \mathrm{Fe}])$, classically allowing us to distinguish CEMP-s, CEMP-rs, and CEMP-r stars. The plot includes stars from this paper and from the literature. Small circles represent our original assignment (Table 1, Col. 8). This classification is not as robust as one could wish. While $r$-stars occupy a distinct region, the exact limit between CEMP-s and CEMP-rs is difficult to define without a certain degree of arbitrariness. Moreover, this classification relies on only two species whose elemental abundances are derived from a small number of lines (Ba II 4554, 5853, and/or $6141 \AA$; Eu II 4129, 4205, and/or $6645 \AA$ ). It would be desirable to use instead information provided by more chemical elements, as developed below.

Figure 7 presents the $[\mathrm{La} / \mathrm{Eu}]$ versus $[\mathrm{Y} / \mathrm{Eu}]$ abundance ratio for CEMP-s and CEMP-rs objects (according to Col. 8 of Table 1). A clear correlation is visible, showing that $[\mathrm{Y} / \mathrm{Eu}]$ is almost as good as $[\mathrm{La} / \mathrm{Eu}]$ (and as $[\mathrm{Ba} / \mathrm{Eu}]$ ) for distinguishing CEMP-rs from CEMP-s stars.

We also tried to find whether an alternative to the $r$-process element europium could improve the separation between classes of stars. Figure 8, displaying [La/Fe] versus $[\mathrm{Sm} / \mathrm{Fe}]$ and $[\mathrm{Gd} / \mathrm{Fe}]$ ( $\mathrm{Sm}$ and Gd being two elements mostly produced by the $r$-process), is thus comparable to Figs. 5 and 6 . The difference between CEMP-s and CEMP-rs is slightly less clear than in the $[\mathrm{La} / \mathrm{Fe}]$ versus $[\mathrm{Eu} / \mathrm{Fe}]$ plane, but the same trends are visible. It thus suggests that a more robust classification could be obtained using the information from as many elemental abundances as possible.

Here we propose a new classification procedure, based on a distance from the solar $r$-process abundance profile. The $r$-process abundance profile is adopted as a reference because the observation of some universality pattern has been invoked in many low-metallicity $r$-process-rich stars (Roederer et al. 2010). For example, the $s$-process is known to be dependent on metallicity, on the environment (radiative or convective), and on the details of the partial mixing of protons into the $\mathrm{C}$-rich radiative intershell region at the time of the third dredge-up. It is thus impossible to define a universal $s$-process abundance profile. On
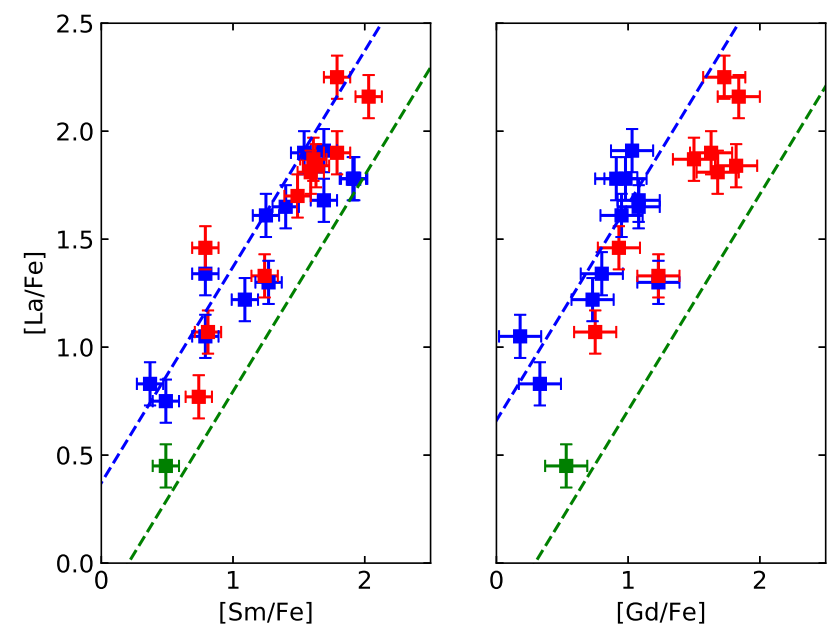

Fig. 8. Same as Fig. 6, but for $[\mathrm{La} / \mathrm{Fe}]$ vs $[\mathrm{Sm} / \mathrm{Fe}]$ (left panel) and [La/Fe] vs [Gd/Fe] (right panel). Symbols are as in Fig. 7 but the stars are now classified according to the new classification (Col. 9 of Table 1).

the contrary, although variations in the $r$-process distribution are observed (Goriely \& Arnould 1997; Sneden et al. 2010; Roederer et al. 2010; Roederer 2011), it appears less sensitive to its operation conditions, and is thus adopted here as a reference baseline.

We compute a signed distance

$d_{\mathrm{S}}=\frac{1}{N} \sum_{x_{i}}\left(\log _{10} \epsilon_{x_{i}, *}-\log _{10} \epsilon_{x_{i}, \operatorname{norm}(r, *)}\right)$

and an rms distance

$d_{\mathrm{rms}}=\left(\frac{1}{N} \sum_{x_{i}}\left(\log _{10} \epsilon_{x_{i}, *}-\log _{10} \epsilon_{x_{i}, \operatorname{norm}(r, *)}\right)^{2}\right)^{1 / 2}$,

where $\left\{x_{1} \ldots x_{N}\right\}$ is the list of the $N$ considered heavy elements, and we use the usual notation $\log _{10} \epsilon_{x_{i}}=\log _{10}\left(n_{x_{i}} / n_{\mathrm{H}}\right)+12$, with $n_{x_{i}}$ the number density of element $x_{i}$. We denote $\log _{10} \epsilon_{x_{i}, *}$ the abundance of element $x_{i}$ as measured in the stars of the present paper (Table B.2), and $\log _{10} \epsilon_{x_{i}, \text { norm }(r, *)}$ the standard $r$-process abundance profile $\log \epsilon_{x_{i}, r}$ normalized to the star abundance profile with respect to europium:

$\log \epsilon_{x_{i}, \operatorname{norm}(r, *)}=\log \epsilon_{x_{i}, r}+\left(\log \epsilon_{\mathrm{Eu}, *}-\log \epsilon_{\mathrm{Eu}, r}\right)$.

The adopted $r$-process abundances $\log \epsilon_{x_{i}, r}$ are listed in Table B.4. We adopted europium as a normalizing element because it is mainly $r$-process; it is also easily measurable in most stars.

Here we consider the element set $x_{i}=\{\mathrm{Y}, \mathrm{Zr}, \mathrm{Ba}, \mathrm{La}, \mathrm{Ce}$, $\mathrm{Nd}, \mathrm{Sm}\}$ because it is the largest intersection of available heavyelement abundances derived in the present paper. The europium abundance is also used, but since it is the normalizing element, its corresponding distance to the $r$-process is null by definition. Actually, the europium abundance could not be determined for HD 55496 (it was previously identified as a CEMP-s star; see Table 1). To determine its new classification, we assign the average $[\mathrm{Eu} / \mathrm{Sm}]$ ratio of the stars previously classified as $s$-enriched in our sample $\left([\mathrm{Eu} / \mathrm{Sm}]_{\mathrm{av}, \mathrm{s}}=-0.42\right)$, leading to $\log \epsilon_{\mathrm{Eu}}=$ -1.63 dex for HD 55496. We note that even if we had assigned the average $[\mathrm{Eu} / \mathrm{Sm}]$ of CEMP-rs stars $\left([\mathrm{Eu} / \mathrm{Sm}]_{\mathrm{av}, \mathrm{rs}}=0.01\right)$, HD 55496 would still be classified as CEMP-s by our new procedure, with $d_{\mathrm{S}}=0.9$ (and $d_{\mathrm{rms}}=1.0$ ) well above the $d_{\mathrm{S}}=0.6$ threshold. 
We can interpret the two distances $d_{\mathrm{S}}$ and $d_{\mathrm{rms}}$ as average abundance distances (in dex) between the abundance profile of a given star and the standard-r abundance profile. Histograms of these two distances are presented in Figs. 9 and 10. The three groups of stars are nicely separated when using either $d_{\mathrm{S}}$ or $d_{\text {rms }}$ distances. As expected, the $r$ stars have the smallest distances, CEMP-rs stars are characterized by intermediate distances $\left(0.5 \leq d_{\text {rms }}<0.8\right)$, and CEMP-s stars have the largest distances from the $r$-process $\left(0.7 \leq d_{\mathrm{rms}} \leq 1.4\right)$.

The advantage of this new classification is that it uses eight abundances instead of two to assign a star to a given class. Because the rms distance considers the observed minus computed $(\mathrm{O}-\mathrm{C})$ absolute values, and as such erases any information contained in the sign of this difference, we decided to adopt in the following the signed distance, and $d_{\mathrm{S}}=0.6$ as the limit between CEMP-s and CEMP-rs stars (represented by the dashed line in Fig. 9). This new assignment is listed as "New class." in Col. 9 of Table 1, and is hereafter adopted in all figures in this paper.

Our new classification confirms the previous assignments except for two borderline objects, HE 1429-0551 and HE 21441832, previously classified as CEMP-rs stars, and now as CEMPs objects. Moreover, CS 22887-048 was classified as a CEMP-rs star by Masseron et al. (2010), but both the new [La/Eu] derived in the present paper and our new classification scheme agree to tag this object as a CEMP-s star.

In Figs. 5 and 6, the overlap between our sample of CEMPs stars and those collected from the literature is very good. We note, however, that our CEMP-s stars extend to higher $s$-process enrichments, especially CS 22887-048 and HE 0111-1346 with $[\mathrm{La} / \mathrm{Fe}] \sim 2$. The $[\mathrm{La} / \mathrm{Fe}]$ of CEMP-rs stars encompass those of CEMP-s stars, but they are located at higher $[\mathrm{Eu} / \mathrm{Fe}]$ values for similar $[\mathrm{La} / \mathrm{Eu}]$ enrichments. In the literature CEMP-rs stars are restricted to very high $[\mathrm{La} / \mathrm{Fe}]$ and $[\mathrm{Eu} / \mathrm{Fe}]$ enrichments (higher than 1.5 and 1 dex, respectively), our sample of CEMP-rs stars extends to lower $[\mathrm{La} / \mathrm{Fe}]$ and $[\mathrm{Eu} / \mathrm{Fe}]$ ratios (Fig. 6). One reason for this is that the $\mathrm{Eu}$ abundance was not systematically determined, and as a consequence these stars were in most cases included in the CEMP-s group based on the enrichment of $s$-process elements; it was not realized that this pattern was merely a consequence of a strong $r$-process enrichment. The case of the well-known CH star HD 5223 is a good example of this difficulty. Since the abundance of europium or of any other $r$-process element was not available for this object, it was previously considered a CEMP-s star. In the present paper we derive $[\mathrm{Ba} / \mathrm{Eu}]=0.24$ and $[\mathrm{La} / \mathrm{Eu}]=0.28$ for this object, making it a definite CEMP-rs object, as confirmed by its $d_{\mathrm{S}}$ value of 0.471 . It also seems that europium was determined only in the most enriched CEMP-s stars, while in our study we systematically undertook its determination even in objects with low enrichment levels.

For CEMP-r stars the $d_{\text {rms }}$ index allows a clearer separation than the $d_{\mathrm{S}}$ index, which was favoured because it contains information on the sign of the $\mathrm{O}-\mathrm{C}$, as explained above. We note, however, that some CEMP-rs objects have abundance profiles resembling those of CEMP-r stars. For example, the CEMP-rs object HD 187861 has $d_{\mathrm{S}}=0.037$, while showing very large overabundances of both $r$ - and $s$-process elements. With $[\mathrm{La} / \mathrm{Eu}]=-0.05$, it is clearly a borderline case between CEMP-rs and CEMP-r stars, as discussed at the beginning of this section.

Another striking result is the abundance continuity between CEMP-s and -rs stars. Drawing a line between CEMP-s and CEMP-rs stars turns out to be somewhat artificial, which explains that several objects are actually borderline cases that
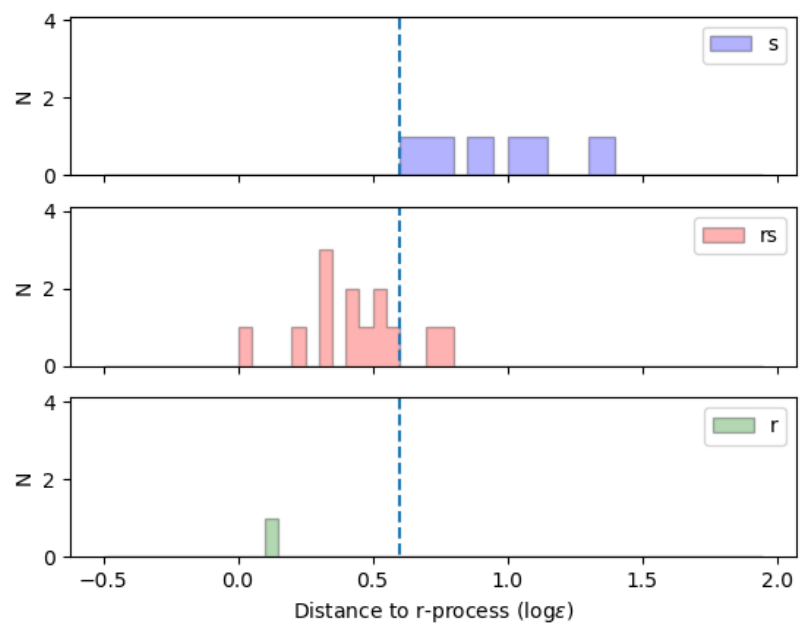

Fig. 9. Histograms of the signed distance $d_{\mathrm{S}}$ (Eq. (3)) for stars classified as CEMP-s stars, CEMP-rs stars, or $r$ star in Col. 8 of Table 1 . The vertical dashed line at $d_{\mathrm{S}}=0.6$ sets the separation between CEMP-s and CEMP-rs stars using our new classification (Col. 9 of Table 1).
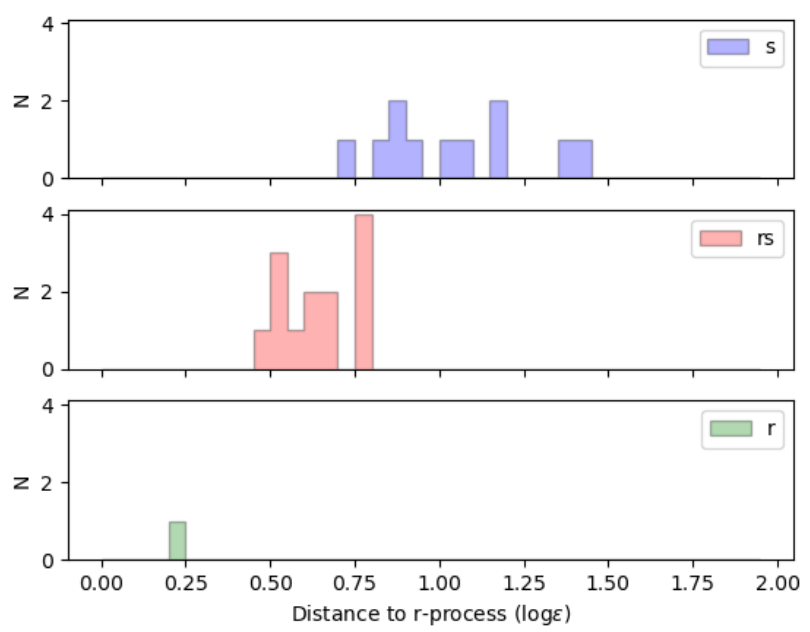

Fig. 10. Histograms of the rms distance (Eq. (4)) separately for stars classified as CEMP-s, CEMP-rs or $r$-star in Col. 8 of Table 1 . Since the re-assignment procedure is based on a threshold value using the signed distance ( $d_{\mathrm{S}}=0.6$, Eq. (3) and Fig. 9), we cannot plot a similar threshold in the present $d_{\mathrm{rms}}$ histogram.

could easily be assigned to either category. Therefore, we note that the new limit drawn between CEMP-rs and CEMP-s classes at $d_{\mathrm{S}}=0.6$ is subject to a certain degree of arbitrariness, especially since we do not know yet whether these two classes originate from different physical mechanisms or represent two extreme manifestations of the same physical process. To answer this question we need to separate the two groups and to investigate their respective properties, as we do in Sect. 8 after discussing individual objects in Sect. 6 and the nucleosynthetic models in Sect. 7.

\section{Discussion on individual stars and comparison with previous abundance studies}

In this section we compare the results from the present abundance analysis (listed in Table B.2) with previous abundance studies. 
CS 22887-048. Tsangarides (2005) studied this object and estimated the atmospheric parameters $T_{\text {eff }}, \log g$, and $[\mathrm{Fe} / \mathrm{H}]$ as $6500 \mathrm{~K}, 3.35$, and -1.70 , respectively, in close agreement with our values with the exception of metallicity for which we derived $[\mathrm{Fe} / \mathrm{H}]=-2.10$. Masseron et al. (2010) classified this object as a CEMP-rs star based on their classification scheme. However, the $[\mathrm{Ba} / \mathrm{Eu}]$ (their paper: 0.51; this paper: 0.42) and [La/Eu] ratios (their paper: 0.24; this paper: 0.50) point to a CEMP-s object. This illustrates the difficulty in distinguishing CEMP-s from CEMP-rs stars using only two or three individual abundances. It justifies our new classification scheme (Sect. 5), which clearly assigns it to the CEMP-s class.

CS 22891-171. This object was analysed by Masseron et al. (2010) who derived atmospheric parameters $T_{\mathrm{eff}}=5100 \mathrm{~K}$, $\log g=1.6$, and $[\mathrm{Fe} / \mathrm{H}]=-2.25$, close to our values of respectively $5215 \mathrm{~K}, 1.24$, and -2.50 . This is yet another object that is a borderline case between the CEMP-s and rs class. Our new classification assigns this star to the CEMP-rs class. In addition to Eu, we report in Table B.2 the first abundances for other $r$-process elements like Gd, Dy, Er, and Hf that strongly support the rs nature of this object.

CS 22942-019. This object was studied by both Aoki et al. (2002) and Masseron et al. (2010), who estimated the parameters $\left(T_{\text {eff }}, \log g,[\mathrm{Fe} / \mathrm{H}]\right)$ as $(5000 \mathrm{~K}, 2.4,-2.64)$ and $(5100 \mathrm{~K}$, $2.5,-2.43)$, respectively. In the present work we obtain $(5100 \mathrm{~K}$, $2.19,-2.50)$, closely matching the parameters from previous studies. This object was classified as a CEMP-s star in both papers. We confirm this classification both with our derived $[\mathrm{La} / \mathrm{Eu}]$ ratio and with our new classification scheme.

CS 30322-023. Various atmospheric parameters have been proposed for this object. Masseron et al. (2006) found $\log g=$ -0.3 , implying that this object could be a thermally pulsing (TP) AGB star (but see the update about this issue in Sect. 8.3). Moreover, its low $\mathrm{C}$ and high $\mathrm{N}$ abundances suggest that it may have experienced hot bottom burning (HBB). However Aoki et al. (2007) found a satisfactory spectral fit with $T_{\text {eff }}=4300 \mathrm{~K}$ and $\log g=1.0$. Our estimated values of $4500 \mathrm{~K}$ and $\log g=1.0$ are closer to those of Aoki et al. (2007) than to those of Masseron et al. (2006). The derived abundances suggest a pure $s$-process pattern in this object, with greater overabundances than those derived by Masseron et al. (2006), a consequence of the difference in their adopted atmospheric parameters. We assign this object to the CEMP-s class based on our new classification criteria.

HD 26. This is a well-known, prototypical $\mathrm{CH}$ star with a clear $s$-process enrichment pattern. It is also a binary with one of the longest orbital periods among the families of extrinsic stars ( $\geq 54 \mathrm{yr}$, could be up to $100 \mathrm{yr}$ ) (Jorissen et al. 2016, 2019), but the orbital parameters still have to be confirmed. Our parameters and abundances are in good agreement with those from previous studies (Van Eck et al. 2003; Vanture et al. 2003; Goswami et al. 2016). Here we add the abundances of Gd, Hf, and Os that were not reported earlier. Carbon is enriched only at a moderate level with $[\mathrm{C} / \mathrm{Fe}]=0.65$. Based on the derived $[\mathrm{La} / \mathrm{Eu}]$ ratio and on our new classification, we confirm the CEMP-s nature of this object.

HD 5223. Goswami et al. (2006) studied this object and estimated the atmospheric parameters as $T_{\text {eff }}=4500 \mathrm{~K}, \log g=1.0$, and $[\mathrm{Fe} / \mathrm{H}]=-2.06$. They derived the abundances of $\mathrm{Ba}, \mathrm{La}$, and $\mathrm{Sm}$ and classified this object as a CEMP-s star. In the present study we derived the abundances of the $r$-process elements $\mathrm{Eu}$,
$\mathrm{Gd}$, Hf, and Os and assigned this object to the CEMP-rs group based on the derived [La/Eu] value. We confirm this assignment with our new classification scheme.

HD 55496. Karinkuzhi \& Goswami (2015) derived $T_{\text {eff }}=$ $4850 \mathrm{~K}, \log g=2.05$, and $[\mathrm{Fe} / \mathrm{H}]=-1.49$ for this star, as well as abundances of $\mathrm{Ba}, \mathrm{Ce}$, and $\mathrm{Pr}$. In the present analysis we estimated the atmospheric parameters to be $4642 \mathrm{~K}, 1.65$, and -2.10 , respectively. Abundances of many $s$-process elements have been obtained for this object, along with the $r$-process elements Sm, $\mathrm{Gd}$, Dy, and Hf. We also found a Pb overabundance of $1.92 \mathrm{dex}$ (NLTE corrected). Light $s$-process elements are more enriched than heavy $s$-process elements in this star, and Fig. 14 shows that this tendency seems to be a distinctive feature of CEMPs stars contrasting with CEMP-rs objects. Because of its very low $[\mathrm{C} / \mathrm{Fe}](=0.07)$ and high $[\mathrm{N} / \mathrm{Fe}](=2.37)$ ratios, HD 55496 actually belongs to the nitrogen-enhanced metal-poor (NEMP) class rather than to the CEMP class. Recently, Pereira et al. (2019) noted its high $[\mathrm{Na} / \mathrm{Fe}]$ and $[\mathrm{Al} / \mathrm{Fe}]$ abundance ratios, similar to those of second-generation globular cluster stars. They suggested that this object, probably polluted by an intermediatemass AGB star that had activated the ${ }^{22} \mathrm{Ne}$ neutron source, evaporated from a globular cluster or from a disrupted dwarf galaxy. We note that there is currently no proof that HD 55496 is a binary (Table 1). Since the Eu abundance has not been measured in this object, the original classification based on the $[\mathrm{La} / \mathrm{Eu}]$ ratio is not possible. However, with our new classification, we assign this object to the CEMP-s group.

$H D$ 76396. This paper presents the first abundance analysis of this object. It shows that HD 76396 is a very metal-poor star $([\mathrm{Fe} / \mathrm{H}]=-2.27)$ with large overabundances for both $s$-process and $r$-process elements. It is definitely a binary with a period of 40 years (Jorissen et al., in prep.), belonging to the CEMP-rs group, as derived from both the original and new classifications.

$H D$ 145777. We present the first abundance analysis of this object, with atmospheric parameters $T_{\text {eff }}=4444 \mathrm{~K}, \log g=0.50$, and $[\mathrm{Fe} / \mathrm{H}]=-2.32$. The star shows a clear enrichment in $s$ process elements (with $[\mathrm{s} / \mathrm{Fe}] \approx 1.0$ ) and in $r$-process elements, albeit to a lower level. It is assigned to the CEMP-rs category based on the derived $[\mathrm{La} / \mathrm{Eu}]$ ratio and on our new classification. Its binarity is unclear.

$H D$ 187861. This object was first studied by Vanture (1992a), and then re-analysed by Van Eck et al. (2003) (as one of their Pb-rich stars) and by Masseron et al. (2010). The range of effective temperatures obtained by these authors covers $\sim 700 \mathrm{~K}$ : while Van Eck et al. (2003) derived $T_{\text {eff }}=5320 \mathrm{~K}, \log g=2.40$, and $[\mathrm{Fe} / \mathrm{H}]=-2.30$, Masseron et al. (2010) obtained $T_{\text {eff }}=$ $4600 \mathrm{~K}, \log g=1.70$, and $[\mathrm{Fe} / \mathrm{H}]=-2.36$. Here we derive an intermediate temperature $\left(T_{\text {eff }}=5000 \mathrm{~K}, \log g=1.50\right.$, and $[\mathrm{Fe} / \mathrm{H}]=-2.60)$. This object shows very large overabundances of both $s$ - and $r$-process elements. We confirm its CEMP-rs nature, both from the derived $[\mathrm{La} / \mathrm{Eu}]$ and the new classification criteria. Its binarity is unknown.

HD 196944. This object was one of the lead-rich stars studied by Van Eck et al. (2003), and also analysed by Aoki et al. (2002), Masseron et al. (2010), and Placco et al. (2015). Our atmospheric parameters are in close agreement with those from these studies, except for $\log g$; we found $\log g=1.28$, while previous analyses found $\log g$ in the range from 1.60 to 1.80 . Based on their criteria, Masseron et al. (2010) and Bisterzo et al. (2011) classified this object as a CEMP-s star. Using nearultraviolet (NUV) spectra from STIS/HST, Placco et al. (2015) 
derived atmospheric parameters $\left(T_{\text {eff }}=5170 \mathrm{~K}, \log g=1.60\right.$, $[\mathrm{Fe} / \mathrm{H}]=-2.41)$ and heavy-element abundances, which they compared with a low-mass low-metallicity model in their Fig. 12. Their $\mathrm{Eu}$ abundance $([\mathrm{Eu} / \mathrm{Fe}]=-0.11)$, lower by at least 0.5 dex compared to all other studies, did not match the model predictions. We obtain a higher $[\mathrm{Eu} / \mathrm{Fe}]$ ratio (after NLTE correction) than that of Aoki et al. (2002) (0.78 versus $0.17 \mathrm{dex}$ ), and a similar Er abundance (1.08 versus 0.81 dex). Even though heavy elements are enriched in HD 196944, the level of enrichment is not high and is almost the same for $s$ - and $r$-elements $([\mathrm{La} / \mathrm{Fe}]=0.77,[\mathrm{Ce} / \mathrm{Fe}]=0.89,[\mathrm{Eu} / \mathrm{Fe}]=0.78,[\mathrm{Dy} / \mathrm{Fe}]=0.80$, and $[\mathrm{Er} / \mathrm{Fe}]=1.08)$. This is fully consistent with the present classification of this star as a CEMP-rs star. This star is a binary of orbital period $1294 \mathrm{~d}$ (Jorissen et al., in prep.).

HD 198269. This object was analysed by Goswami et al. (2016) and their atmospheric parameters $\left(T_{\mathrm{eff}}=4500 \mathrm{~K}\right.$, $\log g=1.50,[\mathrm{Fe} / \mathrm{H}]=-2.03$ ) are similar to our values, except that we derive $\log g=0.83$. With $[\mathrm{La} / \mathrm{Eu}]=0.55$, this object is on the borderline between the CEMP-s and CEMP-rs families. Although Goswami et al. (2016) do not list an Eu abundance, they derive $[\mathrm{Er} / \mathrm{Fe}]=0.43$ and $[\mathrm{Os} / \mathrm{Fe}]=1.09$. In the present analysis $\mathrm{Eu}, \mathrm{Gd}$, and $\mathrm{Os}$ show a mild enrichment $([\mathrm{Eu} / \mathrm{Fe}]=0.67$, $[\mathrm{Gd} / \mathrm{Fe}]=0.73$, and $[\mathrm{Os} / \mathrm{Fe}]=0.55)$, while Dy and Er are highly enriched with $[\mathrm{X} / \mathrm{Fe}]$ values above 1.0 , as are $\mathrm{Ba}, \mathrm{La}, \mathrm{Ce}$, and $\mathrm{Pb}$. This star falls in the CEMP-s category for both the original and new classification schemes. The star has an orbital period of $1295 \mathrm{~d}$.

HD 201626. This object was first analysed by Vanture (1992b) and subsequently by Karinkuzhi \& Goswami (2014). These studies derived the abundances of $s$-process elements only. Here we derive again the $s$-process abundances, and extend the analysis to $r$-process elements. Our atmospheric parameters and abundances are consistent with previous values. With a [La/Eu] ratio of 0.93 , this object naturally falls in the CEMP-s category. We confirm this assignment with our new classification scheme. The star has an orbital period of $1465 \mathrm{~d}$.

HD 206983. Masseron et al. (2010) analysed this object and derived the parameters $\left(T_{\text {eff }}=4200 \mathrm{~K}, \log g=0.60\right.$, $[\mathrm{Fe} / \mathrm{H}]=-1.00)$, which are very similar to ours. This object is only midly enriched in carbon $([\mathrm{C} / \mathrm{Fe}]=0.42)$. The $s$-process elements similarly show a moderate enrichment of $\approx 0.75 \mathrm{dex}$. The Masseron et al. (2010) abundances are similar to ours except for $\mathrm{Pb}$. We derive a $[\mathrm{Pb} / \mathrm{Fe}]$ ratio of 0.88 dex considering the NLTE correction, while Masseron et al. (2010) found a high $\mathrm{Pb}$ enrichment $([\mathrm{Pb} / \mathrm{Fe}]=1.49 \mathrm{dex})$. This star is assigned to the CEMP-s class. It may be a binary.

HD 209621. This object has been studied by Goswami \& Aoki (2010). Their atmospheric parameters $\left(T_{\mathrm{eff}}=4500 \mathrm{~K}\right.$, $\log g=2.0$, and $[\mathrm{Fe} / \mathrm{H}]=-1.93$ ) are slightly different from ours $\left(T_{\text {eff }}=4740 \mathrm{~K}, \log g=1.75\right.$, and $\left.[\mathrm{Fe} / \mathrm{H}]=-2.00\right)$. We find high levels of enrichment for both $s$ - and $r$-process elements. This is thus another CEMP-rs star confirmed with both classification schemes. Its orbital period is $407.4 \mathrm{~d}$.

HD 221170. This well-studied $r$-process star was selected as a comparison object (carbon is not enriched in this apparently non-binary object). Comparing our results with the most recent detailed abundance study by Ivans et al. (2006) reveals that our atmospheric parameters $\left(T_{\text {eff }}=4577 \mathrm{~K}, \log g=0.77\right.$, and $[\mathrm{Fe} / \mathrm{H}]=-2.40)$ are in close agreement with their values $\left(T_{\text {eff }}=4510 \mathrm{~K}, \log g=1.00\right.$, and $\left.[\mathrm{Fe} / \mathrm{H}]=-2.19\right)$.
HD 224959. This is another of the lead-rich stars of Van Eck et al. (2003). Masseron et al. (2010) also analysed this object and obtained $T_{\text {eff }}=4900 \mathrm{~K}, \log g=2.0$, and $[\mathrm{Fe} / \mathrm{H}]=-2.06$. Our estimates are similar for $T_{\text {eff }}(4969 \mathrm{~K})$ and $[\mathrm{Fe} / \mathrm{H}](-2.36)$, but not for $\log g(1.26 \pm 0.3)$. With a $[\mathrm{La} / \mathrm{Eu}]$ ratio of 0.17 , HD 224959 belongs to the CEMP-rs group, in agreement with our new classification scheme.

HE 0111-1346. This object was analysed by Kennedy et al. (2011) using low-resolution infrared spectra. These authors derived $T_{\text {eff }}=4651 \mathrm{~K}, \log g=1.08$, and $[\mathrm{Fe} / \mathrm{H}]=-1.91$ as well as $\mathrm{C}, \mathrm{N}$, and $\mathrm{O}$ abundances. Using these values, Hansen et al. (2016a) derived the $\mathrm{Ba}$ abundance. We present the first detailed chemical abundance pattern. HE 0111-1346 falls in the CEMP$\mathrm{s}$ group using both the original and new classifications. It has an orbital period of $402.7 \mathrm{~d}$.

HE 0151-0341. This star was also analysed by Kennedy et al. (2011), who derived $T_{\text {eff }}=4849 \mathrm{~K}, \log g=1.42$, and $[\mathrm{Fe} / \mathrm{H}]=-2.46$, and Hansen et al. (2016a), who adopted the same parameters. Although our $T_{\text {eff }}$ and $\log g$ agree with their analyses, we find a lower metallicity of -2.89 . Both $s$-process and $r$-process elements are highly enriched: this is another CEMP-rs star in both classifications. It has an orbital period of $359 \mathrm{~d}$.

HE 0319-0215. This is another object analysed by Kennedy et al. (2011) and Hansen et al. (2016a). Kennedy et al. (2011) derived $T_{\text {eff }}=4416 \mathrm{~K}, \log g=0.64$, and $[\mathrm{Fe} / \mathrm{H}]=-2.42$. Our effective temperatures and metallicities are slightly different: $T_{\text {eff }}=4738 \mathrm{~K}, \log g=0.66$, and $[\mathrm{Fe} / \mathrm{H}]=-2.90$. Hansen et al. (2016a) used $T_{\text {eff }}$ and $\log g$ determined by Kennedy et al. (2011) to derive $\mathrm{C}, \mathrm{Fe}$, and $\mathrm{Ba}$ abundances. They found a moderate $[\mathrm{Ba} / \mathrm{Fe}]$ abundance ratio of $0.52 \mathrm{dex}$. Our abundances indicate high enrichment levels for $s$-process and $r$-process elements, reflecting that HE 0319-0215 falls in the CEMP-rs group for both classification schemes. It has an orbital period of $3078 \mathrm{~d}$.

HE 0507-1653. Aoki et al. (2007) derived the atmospheric parameters $\left(T_{\text {eff }}=5000 \mathrm{~K}, \log g=2.4\right.$, and $\left.[\mathrm{Fe} / \mathrm{H}]=-1.50\right)$ well in line with ours. While we find $s$-process elements to be highly enriched (with $[\mathrm{s} / \mathrm{Fe}] \approx 1.75$ ), $r$-process elements are also enhanced with an average $[\mathrm{r} / \mathrm{Fe}]$ ratio of $1.0 \mathrm{dex}$. However, with $[\mathrm{La} / \mathrm{Eu}]=0.65$, this object satisfies the condition to be a CEMP$\mathrm{s}$ star, as confirmed by the new classification. It has an orbital period of $404 \mathrm{~d}$.

HE 1120-2122. Based on a series of low-resolution spectral characteristics (the strength of the $\mathrm{CH} G$-band, the strength of the Ca I feature at $4226 \AA$ and the weakness of the CN band at $4215 \AA$ ) and in comparison with the spectrum of the well-known CH star HD 209621, Goswami et al. (2010) classified this object as a $\mathrm{CH}$ star. The two objects show similar spectral features. There are no former published atmospheric parameters or abundances for this star, which turns out to be a CEMP-rs object for both classification schemes. It has an orbital period of $2103 \mathrm{~d}$.

HE 1429-0551. Aoki et al. (2007) derived $T_{\text {eff }}=4700 \mathrm{~K}$, $\log g=1.5$, and $[\mathrm{Fe} / \mathrm{H}]=-2.50$, close to our values, and presented the abundances of some light elements along with the $\mathrm{Ba}$ abundance. We derived abundances for $\mathrm{Ba}, \mathrm{La}, \mathrm{Ce}, \mathrm{Pr}, \mathrm{Nd}$, $\mathrm{Sm}, \mathrm{Eu}$, and $\mathrm{Gd}$. This is a borderline object, previously classified as CEMP-rs (according to its [La/Eu]), that we re-assign to the CEMP-s class based on our multi-element classification scheme.

HE 2144-1832. Hansen et al. (2016b) derived the atmospheric parameters $T_{\mathrm{eff}}=4200 \mathrm{~K}, \log g=0.6$, and $[\mathrm{Fe} / \mathrm{H}]=-1.7$, 
along with the abundances of $\mathrm{Sr}$ and $\mathrm{Ba}$, and suggest it might be an AGB star. Our parameters match closely. Even though the derived $[\mathrm{La} / \mathrm{Eu}]$ ratio points towards the CEMP-rs category, our new classification scheme assigns it to the CEMP-s class. The carbon abundance from both our and previous analyses indicates that HE 2144-1832 is not much enriched in carbon $([\mathrm{C} / \mathrm{Fe}]=0.77)$.

HE 2255-1724. From the inspection of a low-resolution spectrum of this object, Goswami et al. (2010) identified this object as a $\mathrm{CH}$ star, for which we present the first detailed abundance pattern. Although $r$-process elements (like Sm, Gd, Dy, Er, Os) are enriched in this object, the $[\mathrm{La} / \mathrm{Eu}]$ ratio of 0.54 makes this object a borderline case. We assign it to the CEMP-s category with our multi-element classification.

\section{Comparison with nucleosynthesis predictions}

\subsection{Models of s-and i-processes in low-mass AGB stars}

Asymptotic giant branch nucleosynthesis predictions have been computed coupling the STAREVOL code (Siess \& Arnould 2008) with an extended $s$-process reaction network of 1091 species and the same input physics as in Goriely \& Siess (2018). The solar abundances are from Asplund et al. (2009), and correspond to a metallicity $Z=0.0134$. To describe the mass-loss rate on the RGB, we use the Reimers (1975) prescription with $\eta_{\mathrm{R}}=0.4$ from the main sequence up to the beginning of the AGB and then switch to the Vassiliadis \& Wood (1993) rate. Dedicated models with an initial mass of 1 and $2 M_{\odot}$ have been computed for the different metallicities measured in the present study, namely $[\mathrm{Fe} / \mathrm{H}]=-1,-2.0,-2.5$, and -3.0 .

In the present calculations a diffusion equation is used to compute the partial mixing of protons in the C-rich layers at the time of the third dredge-up (TDU). We follow Eq. (9) of Goriely $\&$ Siess (2018) and use the same diffusive mixing parameters in our simulations as in Shetye et al. (2019), i.e. $f_{\text {over }}=0.14$, $D_{\text {min }}=10^{7} \mathrm{~cm}^{2} \mathrm{~s}^{-1}$, and $p=1 / 2$, where $f_{\text {over controls the }}$ extent of the mixing, $D_{\min }$ is the value of the diffusion coefficient at the innermost boundary of the diffusive region, and $p$ is an additional free parameter describing the shape of the diffusion profile. As shown in Shetye et al. (2019), this adopted set of diffusion parameters gives rise to early TDU episodes and $s$-process enrichments in stars with masses as low as $1 M_{\odot}$ and compatible with observations.

The diffusion algorithm adopted in STAREVOL triggers a rather strong overshoot mixing below the convective envelope, especially at the time of the TDU (Goriely \& Siess 2018). In the case of low-metallicity $2 M_{\odot}$ AGB stars, a strong $s$-process takes place during the various interpulse phases, leading to a surface enrichment compatible with observations, as seen for the 13 CEMP-s stars in Fig. 11.

Interestingly, this mixing is at the origin of the ingestion of protons inside the first thermal pulse of all our low-metallicity $1 M_{\odot}$ AGB stars, as initially found by Iwamoto et al. (2004). In this event protons are moved downwards by the convective flow to regions of higher temperature $\left(T>10^{8} \mathrm{~K}\right)$ where it is depleted via the reaction ${ }^{12} \mathrm{C}(\mathrm{p}, \gamma){ }^{13} \mathrm{~N}$. The subsequent decay of ${ }^{13} \mathrm{~N}$ to ${ }^{13} \mathrm{C}$ is followed by the reaction ${ }^{13} \mathrm{C}(\alpha, \mathrm{n}){ }^{16} \mathrm{O}$, which produces high neutron densities of up to $N_{n} \approx 10^{14}-10^{15} \mathrm{~cm}^{-3}$ (Choplin et al. 2021). After mixing and ensuing rich nucleosynthesis, the convective pulse is found to merge with the stellar envelope, leading to a strong metallicity enrichment of the surface. This surface pollution in carbon and heavy metals tends to accelerate the mass loss, so that no more thermal pulses occur in the subse- quent evolution along the AGB. More details will be given in a forthcoming paper (Choplin et al. 2021).

In Fig. 12 we compare the final surface enrichments obtained for elements heavier than iron in 1 and $2 M_{\odot}$ AGB stars with metallicity $[\mathrm{Fe} / \mathrm{H}]=-2.5$. Figure 12 illustrates the high surface enrichment encountered after one single nucleosynthesis episode in the $1 M_{\odot}$ AGB star, while 14 sequences of TDUs are needed in the $2 M_{\odot}$ AGB star to reach a La overabundance of 2 dex. After dilution and renormalization of the final surface abundance of the $1 M_{\odot}$ AGB star on the 2 dex La overabundance in the $2 M_{\odot}$ star, Fig. 12 also shows the major difference between the two processes, with higher values by about $0.3-0.5$ dex in the $51 \leq$ $Z \leq 55$ and $Z \geq 62$ (including $\mathrm{Pb}$-Bi) regions in the low-mass star. These overproduction factors in the $1 M_{\odot}$ AGB star result from the higher neutron densities (about $N_{n} \approx 10^{14} \mathrm{~cm}^{-3}$ ), which allow short $\beta$-decay branching points to be bypassed and are, in particular, responsible for a significant production of $\mathrm{Eu}$ with respect to $\mathrm{Ba}$ or La. After renormalization on the same La overabundance, we find proportionally more Eu than $\mathrm{La}$ in the $1 M_{\odot}$ star by about $0.6 \mathrm{dex}\left([\mathrm{La} / \mathrm{Eu}]=1.2\right.$ and 0.6 for the 2 and $1 M_{\odot}$ star, respectively). This is similar to the extra Eu overabundance found in CEMP-rs stars with respect to CEMP-s stars (see e.g. Fig. 6). The strongest overproduction is found for Ta $(Z=73)$ for which, unfortunately, no clean and strong enough line can be accurately detected in the visible wavelength range. The lowmetallicity low-mass AGB stars consequently represent a natural site for CEMP-rs stars, as also shown in greater detail in Fig. 11 for the 11 CEMP-rs stars studied in the present paper. In particular, the ratio of the Ba-La-Ce to the Sm-Eu-Gd overabundances is seen to be well reproduced in both classes of stars in Fig. 11. The large overabundance of $\mathrm{Pb}$ in CEMP-rs stars is also fairly well explained, except for HE 1120-2122 and CS 22891-171 stars (the latter has an uncertain $\mathrm{Pb}$ abundance), which show a rather low $\mathrm{Pb}$ enrichment. The similar $\mathrm{Pb}$-to-Ba ratio found in CEMP-s stars like HE 2144-1832 and HD 206983 (the latter has an uncertain $\mathrm{Pb}$ abundance) also cannot be explained by models; models can reach $[\mathrm{Pb} / \mathrm{hs}] \simeq 0$, but only for lower metallicity stars: $[\mathrm{Fe} / \mathrm{H}]=-3.0$, whereas HE 2144-1832 and HD 206983 have $[\mathrm{Fe} / \mathrm{H}]=-1.85$ and -1 , respectively.

\subsection{Quantitative comparison of measured abundances with predicted values}

The overall accuracy of the model predictions is given in Table 1 where the deviation between observation and model is quantified for each star through the $\chi^{2}$ indicator, defined as in Hampel et al. (2016):

$\chi^{2}=\sum_{X} \frac{\left([\mathrm{X} / \mathrm{Fe}]_{\mathrm{obs}}-[\mathrm{X} / \mathrm{Fe}]_{\mathrm{mod}}\right)^{2}}{\sigma_{\mathrm{X}, \mathrm{obs}}^{2}}$.

Here $[\mathrm{X} / \mathrm{Fe}]_{\mathrm{obs}}$ and $[\mathrm{X} / \mathrm{Fe}]_{\bmod }$ are the measured and STAREVOL overproduction factors, respectively, of a given element $X$, and $\sigma_{\mathrm{X} \text {,obs }}$ is the associated uncertainty on the measured abundance. We consider the eight elements available in all stars, namely $\mathrm{Y}, \mathrm{Zr}, \mathrm{Ba}, \mathrm{La}, \mathrm{Ce}, \mathrm{Nd}, \mathrm{Sm}$, and $\mathrm{Eu}^{1}$.

For CEMP-s stars $\chi^{2}$ ranges between 2.7 and 10.9 with an average value of 6.4 , while for CEMP-rs stars values between 1.3 and 10.6 are obtained with a similar average of 6.1. Similar accuracies are obtained with the parametric canonical $i$-process

\footnotetext{
1 As explained in Sect. 5, the Eu abundance could not be determined for HD 55496, which was assigned the CEMP-s average [Eu/Sm], resulting in $\log \epsilon_{\mathrm{Eu}}=-1.63$ dex for that star.
} 

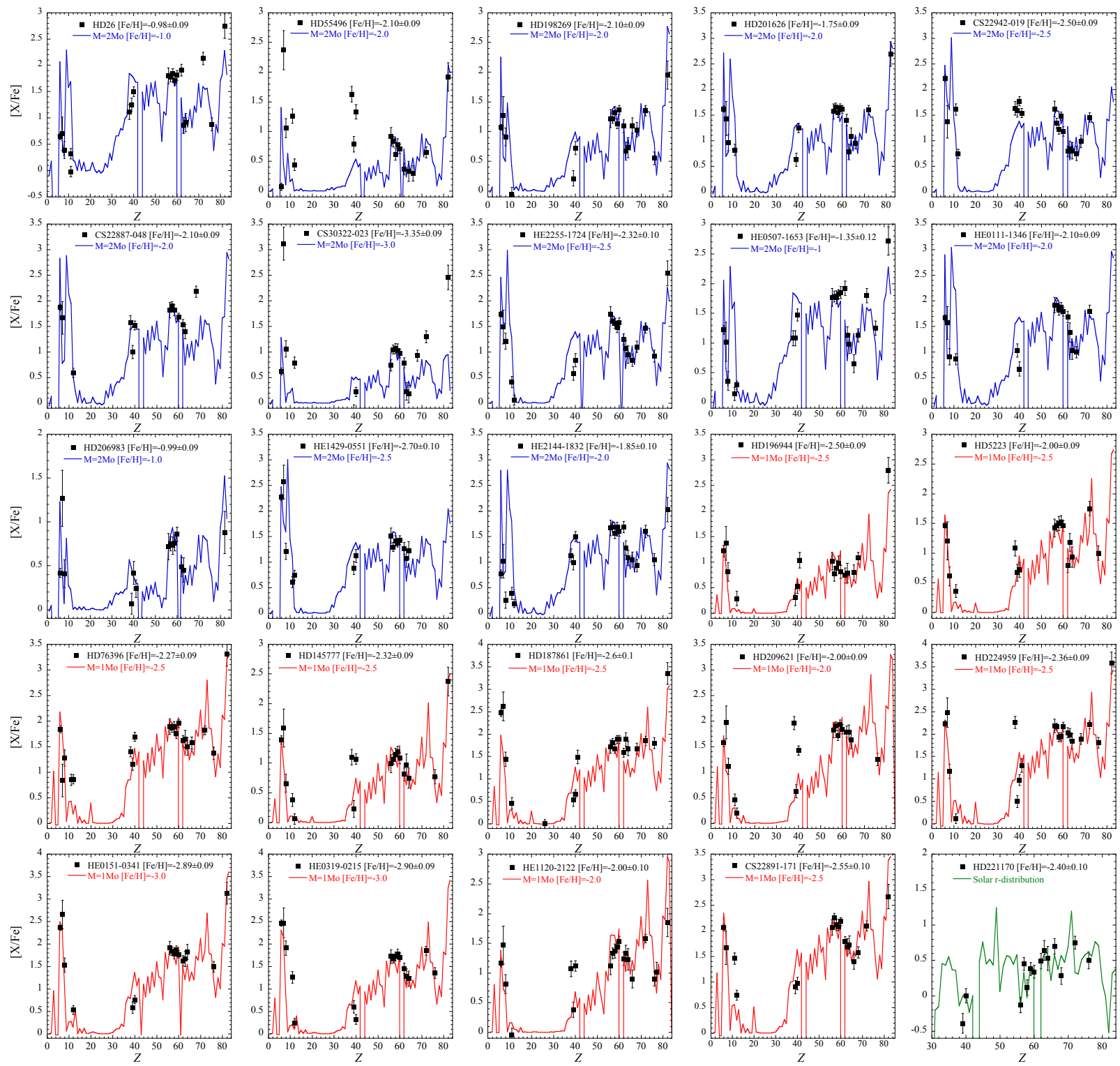

Fig. 11. Comparison of the abundance pattern in CEMP stars with nucleosynthesis predictions from the STAREVOL code. The $2 M_{\odot}$ model predictions for the 13 CEMP-s stars are shown in blue, the $1 M_{\odot}$ predictions for the 11 CEMP-rs stars in red, and the solar $r$-distribution for the sole $r$-star in green. Models are described in Sect. 7.

model developed by Hampel et al. (2016, see in particular their Table 2). We note, however, that in the present stellar evolution models, nucleosynthesis is consistently followed by the realistic stellar evolution model and that the only free parameter is a dilution factor corresponding to the mixing of the nucleosynthesis yields from the primary star into its companion (assumed to be of the same initial composition). In particular, the nucleosynthesis predictions in the $1 M_{\odot}$ models remain insensitive to the mixing parameters considered, and ingestion of protons is found even without imposing any overshoot (Iwamoto et al. 2004; Choplin et al. 2021). This shows that the nucleosynthesis associated with the ingestion of protons in the first thermal pulses of low-mass low-metallicity stars can explain CEMP-rs stars with the same accuracy as that reached by the standard $s$-process in TP-AGB stars (which explains the CEMP-s stars).

In summary, the neutron-capture process taking place in the conditions found here in low-metallicity $1 M_{\odot}$ AGB stars corresponds to an efficient $s$-process with a relatively high neutron density. Consequently, the origin of CEMP-rs stars may not need to call for exceptional astrophysical sites, such as rapidly accreting C-O or O-Ne white dwarfs in a close binary system (Denissenkov et al. 2017) or a complex double s+r enrichment scenario (Abate et al. 2016).

\section{Abundance profile analysis}

\subsection{The $[\mathrm{hs} / \mathrm{ls}]$ ratio}

The second-to-first $s$-process peak abundance ratio ([hs/ls]) and the third-to-second $s$-process peak abundance ratio $([\mathrm{Pb} / \mathrm{hs}])$ give insight on the $s$-process efficiency (where ls and hs stand for $s$-process elements of the first ( $\mathrm{Sr}, \mathrm{Y}, \mathrm{Zr}$ ) and second (Ba, La, Ce) peaks, respectively).

Hollek et al. (2015) noted that the abundance continuum present in CEMP-s and CEMP-rs stars calls for a new classification scheme, and proposed an alternative based on the $[\mathrm{Y} / \mathrm{Ba}]$ value. Here we discuss $[\mathrm{Ba} / \mathrm{Y}]$ to better match the usual $[\mathrm{hs} / \mathrm{ls}]$ index. Hollek et al. (2015) separated CEMP stars into three 


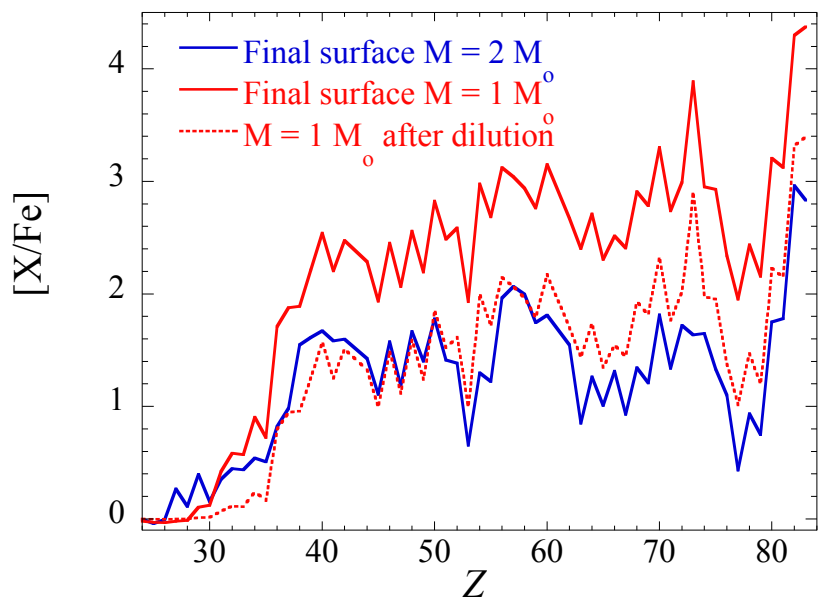

Fig. 12. Comparison of the final elemental surface distributions $[\mathrm{X} / \mathrm{Fe}]$ (solid lines) obtained after the development of the neutron-capture processes in $2 M_{\odot}$ (blue line) and $1 M_{\odot}$ (red line) AGB stars of metallicity $[\mathrm{Fe} / \mathrm{H}]=-2.5$. The dashed red line is obtained after dilution of the final surface abundances of the $1 M_{\odot}$ star with a solar-like abundance distribution in order to reproduce the 2 dex $\mathrm{La}(Z=57)$ overabundance of the $2 M_{\odot}$ star. This model is called for to explain the CEMP-s stars, while the $1 M_{\odot}$ model explains the CEMP-rs stars, as shown in Fig. 11 .

groups of increasing $[\mathrm{hs} / \mathrm{ls}]$ : CEMP-sA $(0.3<[\mathrm{Ba} / \mathrm{Y}]<0.9)$, CEMP-sB $(0.9<[\mathrm{Ba} / \mathrm{Y}]<1.5)$, and CEMP-sC $([\mathrm{Ba} / \mathrm{Y}]>1.5)$. They concluded that their CEMP-sA group mainly concerns CEMP-s stars, whereas CEMP-rs stars belong mostly to the CEMP-sC group.

Figure 13 presents $[\mathrm{Ba} / \mathrm{Y}]$ and $[\mathrm{La} / \mathrm{Y}]$ as a function of [La/Eu]. There is a slight offset of the [hs/ls] distribution of CEMP-rs stars (shifted to higher values) with respect to that of CEMP-s stars. This distribution offset is most clearly seen when using $[\mathrm{Ba} / \mathrm{Y}]$.

This offset is tiny, but clearly seen in the boxplots in Fig. 14: both the median (indicated as a horizontal line in the blue and red boxes) and the quartiles are shifted, always in the same direction for two light-s elements $\left([1 \mathrm{~s} / \mathrm{Fe}]_{\text {CEMP-s }}>[1 \mathrm{~s} / \mathrm{Fe}]_{\text {CEMP-rs }}\right)$ and for four heavy-s elements ([hs/Fe $\left.]_{\text {CEMP-s }}<[\mathrm{hs} / \mathrm{Fe}]_{\text {CEMP-rs }}\right)$. This effect can be partly ascribed to metallicity: though the [hs/ls] ratio does not show an extremely marked trend with metallicity (see middle left panel of Fig. 15), the seven objects with $[\mathrm{hs} / \mathrm{ls}]>1$ all have $[\mathrm{Fe} / \mathrm{H}]<-2$. However, metallicity alone is not responsible for the offsets in Fig. 14, since the [hs/ls] distributions (where $[\mathrm{Fe} / \mathrm{H}]$ cancels) of CEMP-rs and CEMP-s stars are shifted in both Figs. 13 and 15. We note that Fig. 15 presents a more robust estimate of $[\mathrm{hs} / \mathrm{ls}]$, with $[\mathrm{hs} / \mathrm{Fe}]$ computed as $0.5 \times([\mathrm{La} / \mathrm{Fe}]+[\mathrm{Ce} / \mathrm{Fe}])$ and $[1 \mathrm{~s} / \mathrm{Fe}]$ as $0.5 \times([\mathrm{Y} / \mathrm{Fe}]+[\mathrm{Zr} / \mathrm{Fe}])$. In conclusion, Figs. 13-15 show that there is a tendency for CEMP-rs stars to have higher [hs/ls] ratios than CEMP-s stars.

In order to assess whether the difference between the [hs/ls] distributions of CEMP-s and CEMP-rs stars is significant, we performed a Wilcoxon rank-sum test (also called the MannWhitney U test), which allows us to determine whether two independent samples are selected from populations having the same distribution. Let $H_{0}$ be the null hypothesis that the two groups of measured [hs/ls] ratios for CEMP-rs and CEMP-s stars are drawn from the same distribution. Based on the values listed in Table 3 , the risk of rejecting $H_{0}$ even while it is true is $2.6 \%$. Therefore, the null hypothesis $H_{0}$ of population identity for [hs/ls] can be rejected with a high confidence level. For the sake of completeness, the same rank test has also been
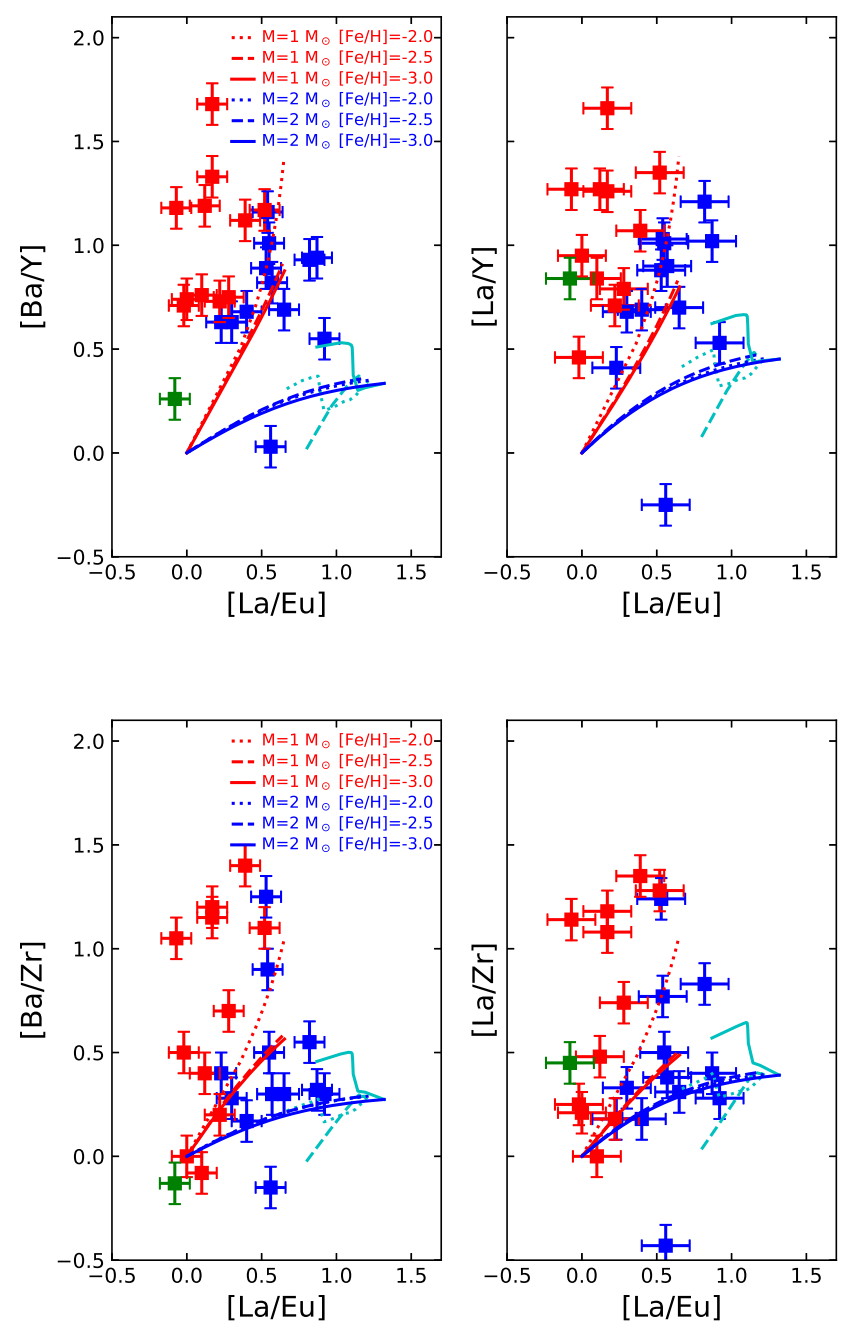

Fig. 13. Four $[\mathrm{hs} / \mathrm{ls}$ ] indices (where $\mathrm{ls}=\mathrm{Zr}$ or $\mathrm{Y}$, and $\mathrm{hs}=\mathrm{Ba}$ or $\mathrm{La}$ ) as a function of the $[\mathrm{s} / \mathrm{r}]$ index $[\mathrm{La} / \mathrm{Eu}]$. Symbols are as in Fig. 7. Theoretical predictions for 1 and $2 M_{\odot}$ AGB stars at different metallicities, as labelled, are overplotted. For the $2 M_{\odot}$ models the cyan line represents the evolution of the abundances along the AGB, whereas the blue and red lines represents the dilution of tip-of-the-AGB material in the envelope of the companion, ultimately producing material of solar abundance $([\mathrm{Ba} / \mathrm{Zr}]=0,[\mathrm{La} / \mathrm{Eu}]=0,[\mathrm{La} / \mathrm{Zr}]=0)$. Since the $1 M_{\odot}$ models experience a single thermal pulse, the cyan line reduces to a single point in that case and the red lines represent the mere dilution as above for the blue lines. We note that the $1 M_{\odot}$ model predictions at $[\mathrm{Fe} / \mathrm{H}]=-2.5$ and -3 almost exactly superimpose.

applied to individual elemental abundances in order to assess the significance of the differences observed in their distribution displayed in Fig. 14. The risk of rejecting $H_{0}$ even while true is always lower than $15 \%$, except for Sm (Table 3). Considering Pr, $\mathrm{Eu}, \mathrm{Gd}$, and Dy (i.e. mostly $r$-elements), the risk is even lower than $5 \%$.

Therefore, the null hypothesis $H_{0}$ of population identity can be rejected with a high confidence level for both light-s and heavy-s elements. It can be safely concluded that CEMP-rs stars have on average higher $[\mathrm{hs} / \mathrm{Fe}]$ and lower $[\mathrm{ls} / \mathrm{Fe}]$ than CEMP-s stars, and that this difference is statistically significant.

We thus conclude that CEMP-rs stars have a tendency towards higher [hs/ls] (thereby confirming the Hollek et al. 2015 results), higher $[\mathrm{hs} / \mathrm{Fe}]$, and lower metallicity when compared to CEMP-s stars, though a large overlap exists between the 


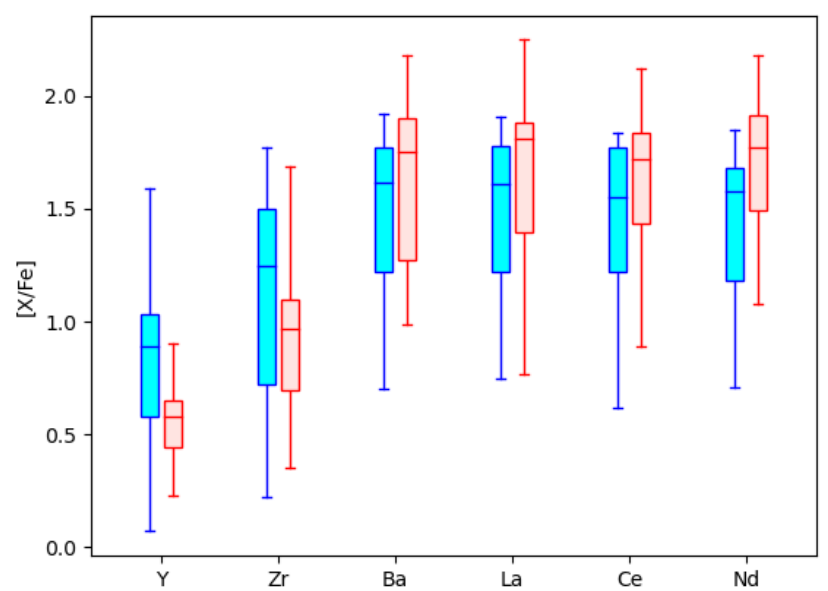

Fig. 14. Abundance distribution of light-s $(X=Y, Z r)$ and heavy-s $(\mathrm{X}=\mathrm{Ba}, \mathrm{La}, \mathrm{Ce}, \mathrm{Nd}$ ) elements for CEMP-s (blue) and CEMP-rs (red) stars. The box extends from the lower to upper $[\mathrm{X} / \mathrm{Fe}]$ quartile, with a small horizontal line at the median. The whiskers extend from the box to show the full range of the data.
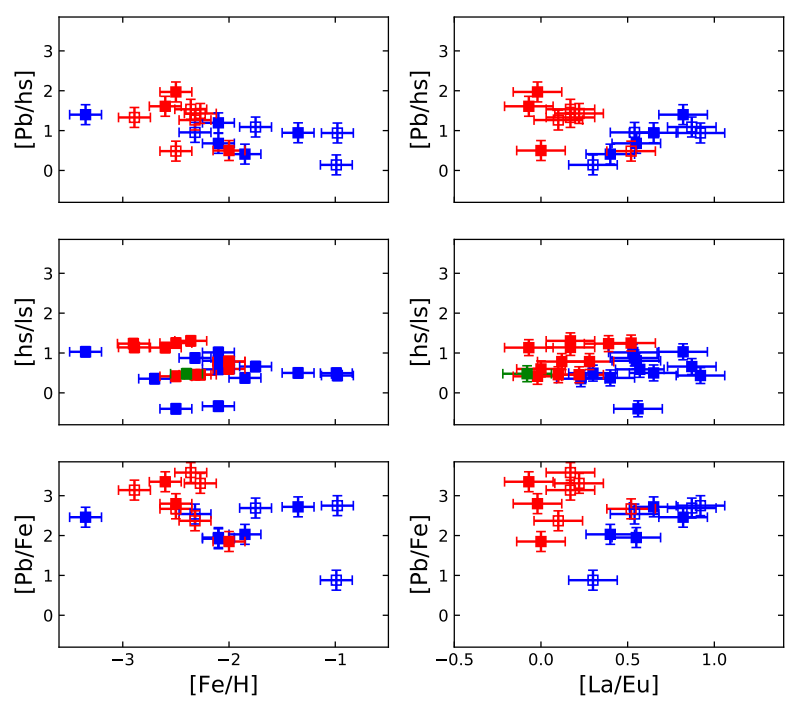

Fig. 15. $[\mathrm{Pb} / \mathrm{hs}]$ and $[\mathrm{hs} / \mathrm{ls}]$ as a function of the metallicity $[\mathrm{Fe} / \mathrm{H}]$ (left column) and of $[\mathrm{La} / \mathrm{Eu}]$ (right column). The [hs/Fe] ratio is computed as $([\mathrm{La} / \mathrm{Fe}]+[\mathrm{Ce} / \mathrm{Fe}]) / 2$ and $[1 \mathrm{~s} / \mathrm{Fe}]$ as $([\mathrm{Y} / \mathrm{Fe}]+[\mathrm{Zr} / \mathrm{Fe}]) / 2$. As usual, blue and red symbols represent CEMP-s and -rs stars, respectively. Empty symbols represent objects with uncertain $\mathrm{Pb}$ abundances, whereas filled symbols represent objects with reliable $\mathrm{Pb}$ abundances. The non-LTE $\mathrm{Pb}$ abundances presented in Table B.2 are used for both cases.

distributions of the two stellar classes. This continuity between CEMP-s and CEMP-rs abundance patterns does not point to a totally different site for the $i$-process nucleosynthesis, but rather to the same physical process as the one producing the classical $s$-process but occurring under slightly different, more extreme conditions (i.e. lower masses, lower metallicities).

Two CEMP-s stars, HD 55496 and CS 22942-019, have a negative [hs/ls] index, implying that they have high $\mathrm{Y}$ and $\mathrm{Zr}$ abundances with respect to $\mathrm{La}$ and $\mathrm{Ce}$. It remains difficult to explain theoretically such a trend within the low-mass AGB star models. In contrast, higher mass AGB stars are known to be subject to a non-negligible neutron irradiation within the thermal pulses that give rise to larger production of light $s$-elements with respect to heavier species (e.g. Goriely \& Siess 2005). Considering an intermediate-mass AGB star would also help to explain the high $\mathrm{N}$ abundance found in HD 55496, as discussed in Sect. 8.3.

In Fig. 13 the model predictions are also overplotted, for the same mass and metallicity range as in Fig. 12. These predictions start at high $[\mathrm{La} / \mathrm{Eu}]$ and $[\mathrm{hs} / \mathrm{ls}]$ values (representing the first TDU), and join the $(0,0)$ point for infinite dilution of TP-AGB enriched material into the (solar-scaled composition) companion envelope composition. In the case of $2 M_{\odot}$ models, a specific colour-coding in Figs. 13, 16, 17, 19, and 20 allows us to distinguish TP-AGB enrichment (in cyan) from dilution (in blue). For the $1 M_{\odot}$ models this distinction was not possible since they experience a single thermal pulse.

Figure 13 nicely shows that the [hs/ls] predicted abundance ratios of the low-mass AGB stars (in red) are higher than those of stars experiencing a standard $s$-process (in blue). However, because of dilution, all models converge to the origin, which explains the large overlap in [hs/ls] and in $[\mathrm{La} / \mathrm{Eu}]$ of both modelled and measured abundances. We note here that the measured [hs/ls] ratios, when represented by $[\mathrm{Ba} / \mathrm{Y}]$, are well above the predictions compared to $[\mathrm{Ba} / \mathrm{Zr}]$. We found no explanation for this offset, but it may also be due to the absence of NLTE correction for the $\mathrm{Y}$ lines used for the analysis.

\subsection{The $P b$ abundance}

We now turn to the lead-to-second $s$-process peak abundance ratio. The $[\mathrm{Pb} / \mathrm{hs}]$ dependence on $[\mathrm{Fe} / \mathrm{H}]$ is displayed in Fig. 15. Non-LTE Pb abundances are plotted with full squares, while uncertain abundances (flagged with ":" in Table B.2) are represented as empty squares. For convenience, the $[\mathrm{s} / \mathrm{Fe}]$, [ls/Fe], [hs/Fe], and [hs/ls] of the programme stars are listed in Table B.3. A trend of increasing [ $\mathrm{Pb} / \mathrm{hs}]$ with decreasing metallicity is observed in this figure. The usual explanation is that the efficiency of the $s$-process increases when metallicity decreases, more neutrons being available per iron seed nuclei.

The ([Pb/hs], $[\mathrm{La} / \mathrm{Eu}])$ panel of Fig. 15 illustrates the clearest separation between CEMP-s and CEMP-rs stars that we could achieve in this paper: CEMP-s stars have high [La/Eu] and low $[\mathrm{Pb} / \mathrm{hs}]$, while CEMP-rs stars are characterized by low $[\mathrm{La} / \mathrm{Eu}]$ and high $[\mathrm{Pb} / \mathrm{hs}]$. The only exception is the CEMPrs star CS 22891-171, which is however one of the objects with uncertain $\mathrm{Pb}$ abundances. This tendency (high $[\mathrm{Pb} / \mathrm{hs}]$ for low $[\mathrm{La} / \mathrm{Eu}]$ ) is somewhat expected because when the La abundance increases, all other things being equal, $[\mathrm{Pb} / \mathrm{hs}]$ will decrease (because hs $=(\mathrm{La}+\mathrm{Ce}) / 2$ ). However, this trend is not observed among CEMP-s stars alone where $[\mathrm{Pb} / \mathrm{hs}]$ increases with increasing $[\mathrm{La} / \mathrm{Eu}]$. It probably reflects the fact that among CEMP-s stars Eu is not produced and has its solar-scaled abundance, so that stars with high second-peak abundances (La), indicating an efficient $s$-process, also tend to have high third-peak abundances $(\mathrm{Pb})$. On the contrary, in CEMP-rs objects, a measurable intrinsic Eu production would disturb this trend by decreasing $[\mathrm{La} / \mathrm{Eu}]$.

\subsection{CNO and heavy elements}

Figure 16 shows the $\mathrm{CNO}$ abundances with $[\mathrm{La} / \mathrm{Eu}]$ and $[\mathrm{Ba} / \mathrm{Eu}]$. The good separation between the CEMP-s and -rs subgroups in the $([\mathrm{C} / \mathrm{N}],[\mathrm{La} / \mathrm{Eu}])$ or $([\mathrm{C} / \mathrm{N}],[\mathrm{Ba} / \mathrm{Eu}])$ planes simply reflects the separating power of $[\mathrm{La} / \mathrm{Eu}]$ or $[\mathrm{Ba} / \mathrm{Eu}]$.

Nitrogen-enhanced metal-poor stars have been defined by Johnson et al. (2007) as CEMP stars with $[\mathrm{C} / \mathrm{N}]<-0.5$ and $[\mathrm{N} / \mathrm{Fe}]>0.5$. According to this definition, two NEMP stars are present in our sample, both also classified as CEMP-s stars: 
Table 3. Results of the Wilcoxon rank-sum test: probability of rejecting, while it would be true, the null hypothesis that the two groups of measured abundances of element $X$ for CEMP-rs and CEMP-s stars are drawn from the same distribution, and the same for the [hs/ls] value, where ls and hs are defined as in Fig. 14.

\begin{tabular}{lcccccccccccc}
\hline \hline Element & $\mathrm{Y}$ & $\mathrm{Zr}$ & $\mathrm{Ba}$ & $\mathrm{La}$ & $\mathrm{Ce}$ & $\mathrm{Pr}$ & $\mathrm{Nd}$ & $\mathrm{Sm}$ & $\mathrm{Eu}$ & $\mathrm{Gd}$ & $\mathrm{Dy}$ & {$[\mathrm{hs} / \mathrm{ls}]$} \\
\hline Probability (\%) & 11.7 & 14.2 & 13.0 & 9.7 & 11.7 & 3.2 & 4.9 & 21.8 & 0.2 & 0.2 & 2.5 & 2.6 \\
\hline
\end{tabular}
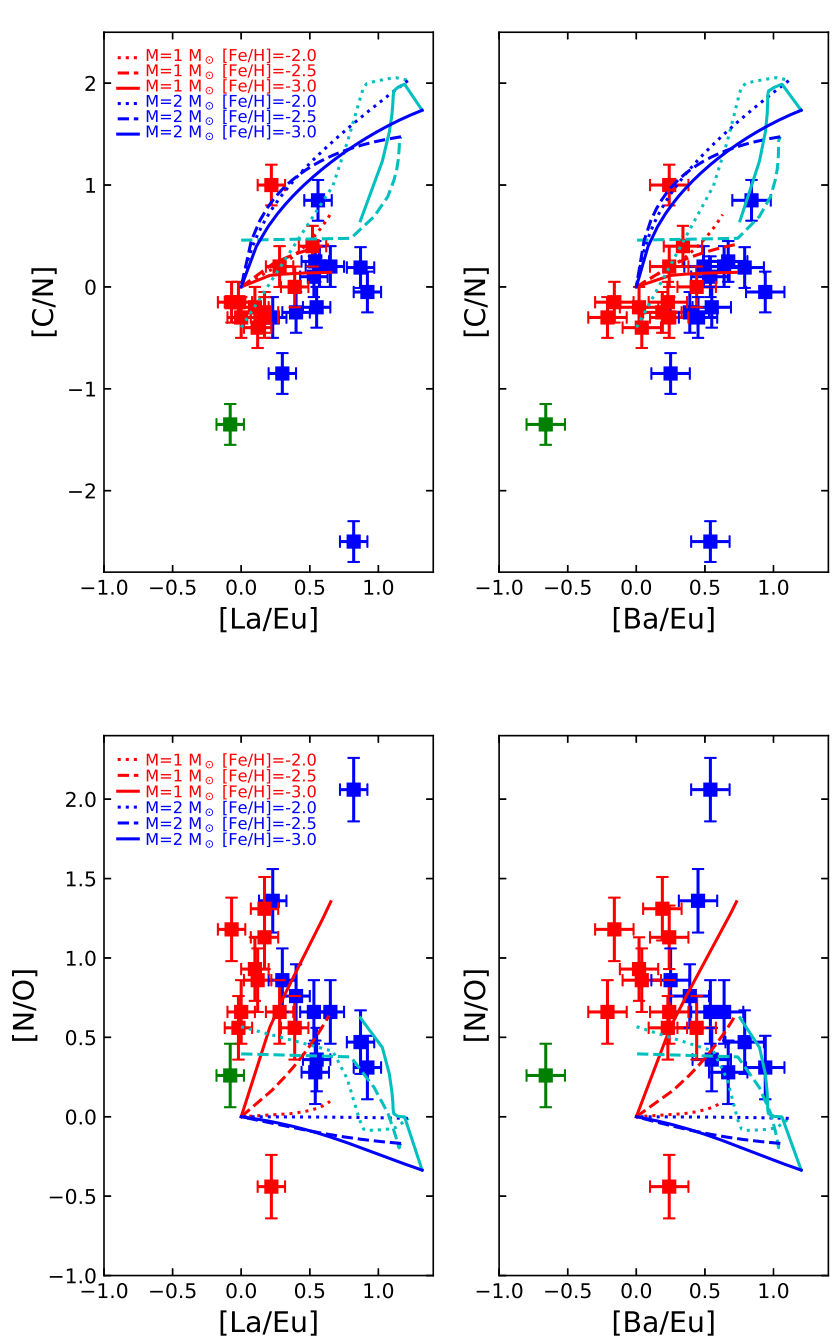

Fig. 16. Abundance ratios of $[\mathrm{C} / \mathrm{N}]$ and $[\mathrm{N} / \mathrm{O}]$ as a function of $[\mathrm{La} / \mathrm{Eu}]$ and $[\mathrm{Ba} / \mathrm{Eu}]$. Symbols are as in Fig. 7, and cyan curves as in Fig. 13.

CS 30322-023 and HD 55496. Unfortunately, the latter has no Eu measurement available, but its $\mathrm{Sm}$ abundance can be used as a proxy. If, as we do in Sect. 5, we assign to HD 55496 the average $[\mathrm{Eu} / \mathrm{Sm}]$ of CEMP-s stars $\left([\mathrm{Eu} / \mathrm{Sm}]_{\mathrm{av}, \mathrm{s}}=-0.42\right)$, we infer $[\mathrm{La} / \mathrm{Eu}]=0.92$ and $[\mathrm{C} / \mathrm{N}]=-2.3$ for HD 55496, which makes it a twin of CS 30322-023 $([\mathrm{La} / \mathrm{Eu}]=0.82,[\mathrm{C} / \mathrm{N}]=-2.5)$. This high nitrogen abundance points towards HBB Boothroyd et al. (1995) in massive AGB stars $\left(M \gtrsim 4 M_{\odot}\right)$, but the positive [hs/ls] ratio of CS 30322-023 does not comply with an $s$-process nucleosynthesis powered by ${ }^{22} \mathrm{Ne}(\alpha, \mathrm{n})^{25} \mathrm{Mg}$ in the convective pulse. The situation is more understandable with HD 55496 where [hs/ls] is negative, as already discussed in Sect. 8.1.

We note that Masseron et al. (2006) interpreted CS 30322023 as a possible low-metallicity TP-AGB star since the distance estimated from the spectroscopic parameters led to a luminosity

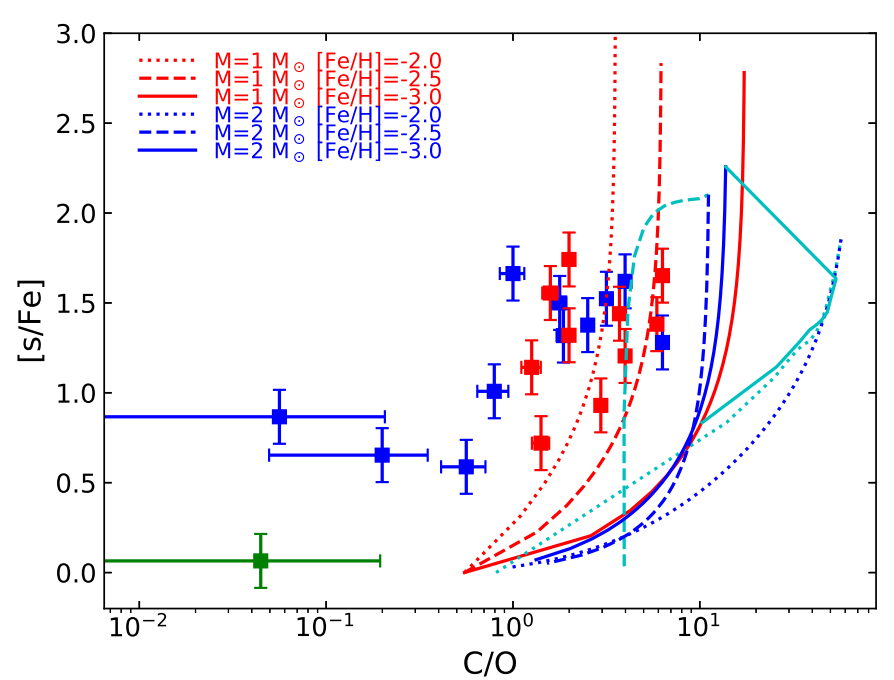

Fig. 17. Surface $s$-enrichment $[\mathrm{s} / \mathrm{Fe}]$ as a function of the surface $\mathrm{C} / \mathrm{O}$, with $[\mathrm{s} / \mathrm{Fe}]$ defined as $([\mathrm{Y} / \mathrm{Fe}]+[\mathrm{Zr} / \mathrm{Fe}]+[\mathrm{Ba} / \mathrm{Fe}]+[\mathrm{La} / \mathrm{Fe}]+[\mathrm{Ce} / \mathrm{Fe}]$ $+[\mathrm{Nd} / \mathrm{Fe}]) / 6$. The measured abundances are colour-coded as in Fig. 7 , and the model predictions as in Fig. 13.

compatible with the TP-AGB in the HR diagram. This interpretation is difficult to reconcile with its high nitrogen abundance implying an intermediate-mass star, if the high nitrogen abundance is indeed due to HBB. With the Gaia DR2 parallax, the position of CS 30322-023 in the HR diagram or in a $\left(\log g, T_{\text {eff }}\right)$ diagram is compatible with that of a tip-of-the-RGB star (see Figs. 23-26, where CS 30322-023 is located at $\log T_{\text {eff }}=3.65$ and $\log g=1$ ).

Considering now the group of objects with $[\mathrm{C} / \mathrm{N}]>-1$ in Fig. 16, there might be a loose trend (among CEMP-s and CEMP-rs stars separately) of increasing [C/N] with increasing $[\mathrm{La} / \mathrm{Eu}]$ (and $[\mathrm{Ba} / \mathrm{Eu}])$. This trend is expected in low-mass stars as third dredge-ups in the absence of $\mathrm{HBB}$ contribute to increase both $s$-process elements and C.

Finally, we note that CEMP-s and CEMP-rs stars do not seem to belong to separate classes as far as their $\mathrm{C}, \mathrm{N}$ and $\mathrm{O}$ abundances are concerned. Instead, they show a continuity in their $[\mathrm{C} / \mathrm{N}]$ and $[\mathrm{N} / \mathrm{O}]$ abundance ratios.

The comparison between model predictions and abundance determinations is shown in Fig. 16. A significant number of CEMP-rs stars have $[\mathrm{C} / \mathrm{N}] \lesssim 0$, a feature that is not reproduced by the low-mass $\left(1 M_{\odot}\right)$ models. Furthemore, the $[\mathrm{C} / \mathrm{N}]$ and $[\mathrm{N} / \mathrm{O}]$ ratios are hardly explained by the more traditional nucleosynthesis in the $2 M_{\odot}$ AGB star.

Figure 17 illustrates the correlation between the surface $s$-process enrichment and the $\mathrm{C} / \mathrm{O}$ ratio. We note that the $1 M_{\odot}$ predictions are in better agreement than the $2 M_{\odot}$ values. The large $\mathrm{C}$ overabundances predicted in AGB stars, but not confirmed by derived abundances, is a long-standing problem (see e.g. Shetye et al. 2019). 


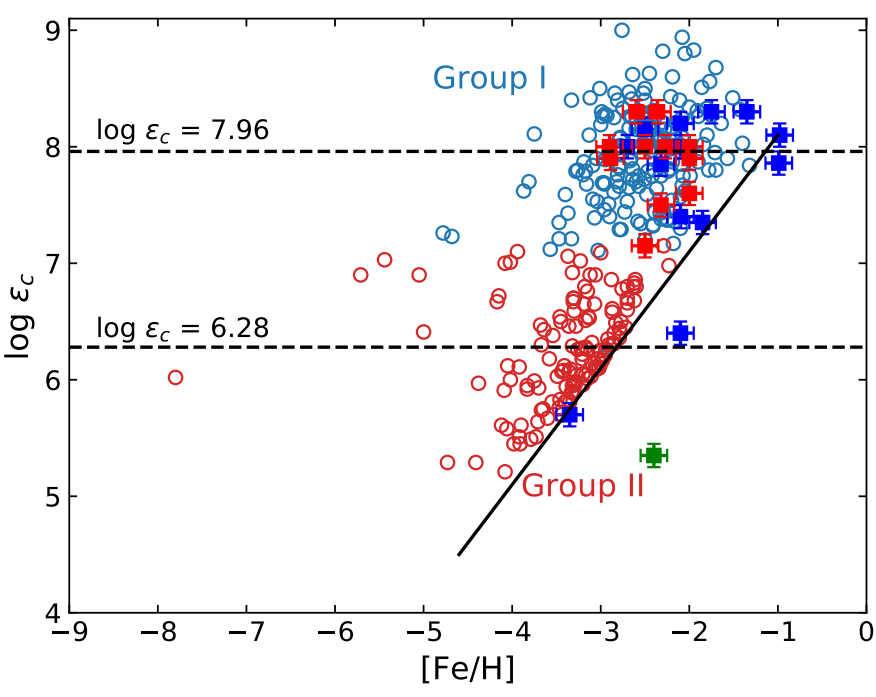

Fig. 18. Scatter plot of the carbon abundance $\left(\log \epsilon_{\mathrm{C}}\right)$ as a function of metallicity. The diagonal line traces the Galactic evolution of carbon with metallicity, in the absence of any in situ enhancement. The stars in this sample are indicated by blue (CEMP-s) and red (CEMP-rs) squares. CEMP stars from the literature as compiled by Yoon et al. (2016) are superimposed: CEMP-s stars are represented as blue circles, they constitute Group I, with an average $\mathrm{C}$ abundance as indicated by the upper dashed horizontal line. CEMP-no stars are plotted as red circles, and define Group II.

A figure displaying $\log \epsilon_{\mathrm{C}}$ as a function of $[\mathrm{Fe} / \mathrm{H}]$ was first presented by Spite et al. (2013) and then further discussed by Hansen et al. (2016c) and Yoon et al. (2016). Here Fig. 18 represents our stellar sample, together with the compilation of CEMPs, -rs, and -no stars of Yoon et al. (2016). It clearly shows the bimodal carbon abundance distribution among CEMP stars, with a high-C group (centred on $\log \epsilon_{\mathrm{C}}=7.96$ ) containing mostly CEMP-s stars and a low-C group (centred on $\log \epsilon_{\mathrm{C}}=6.28$ ) composed of CEMP-no stars. As noted by Yoon et al. (2016), the $\log \epsilon_{\mathrm{C}}$ distribution of the CEMP-rs stars exhibits no clear difference from that of the CEMP-s stars. However, two CEMP-s stars in our sample, not present in the Yoon et al. (2016) sample, surprisingly fall in the low-C (group II) zone, among CEMP-no objects; they are CS 30322-023 and HD 55496, the two NEMP stars where some carbon might have been turned into nitrogen by HBB, as mentioned above. Actually the carbon abundance of HD 55496 falls below the diagonal line tracing the Galactic evolution of carbon with metallicity, in the absence of any in situ enhancement. This sub-Galactic carbon abundance also points to the operation of HBB. To be reconciled with the average carbon abundance of CEMP-s stars $\left(\log \epsilon_{\mathrm{C}}=7.96\right.$, see Fig. 18), CS 30322-023 and HD 55496 lack respectively $\Delta \log \epsilon_{\mathrm{C}}=2.26$ and $1.56 \mathrm{dex}$ of carbon. Given that this amount of carbon is entirely transformed to nitrogen by the $\mathrm{CNO}$ cycle $\left(\Delta \log \epsilon_{\mathrm{N}}=\right.$ $\left.-\Delta \log \epsilon_{\mathrm{C}}\right)$, we have $[\mathrm{C} / \mathrm{N}]_{\text {measured }}=[\mathrm{C} / \mathrm{N}]_{\text {without } \mathrm{HBB}}-2 \Delta \log \epsilon_{\mathrm{C}}$. These two stars have a low $[\mathrm{C} / \mathrm{N}]$ ratio $([\mathrm{C} / \mathrm{N}]=-2.5$ and -2.3 for CS 30322-023 and HD 55496, respectively; see Table B.2). Without $\mathrm{HBB}$, the $[\mathrm{C} / \mathrm{N}]$ ratio of the $\mathrm{AGB}$ progenitors of these two stars would thus be around $[\mathrm{C} / \mathrm{N}]=2$. This is exactly the value predicted for low-mass stars not experiencing $\mathrm{HBB}$ (Johnson et al. 2007), as can also be seen in the upper panel of Fig. 16, top of the cyan curve, prior to dilution (in blue) in the companion envelope. After dilution, the $[\mathrm{C} / \mathrm{N}]$ ratio for these two objects, had they not experienced HBB, would then be fully compatible with that of the bulk of CEMP-s stars. The measured

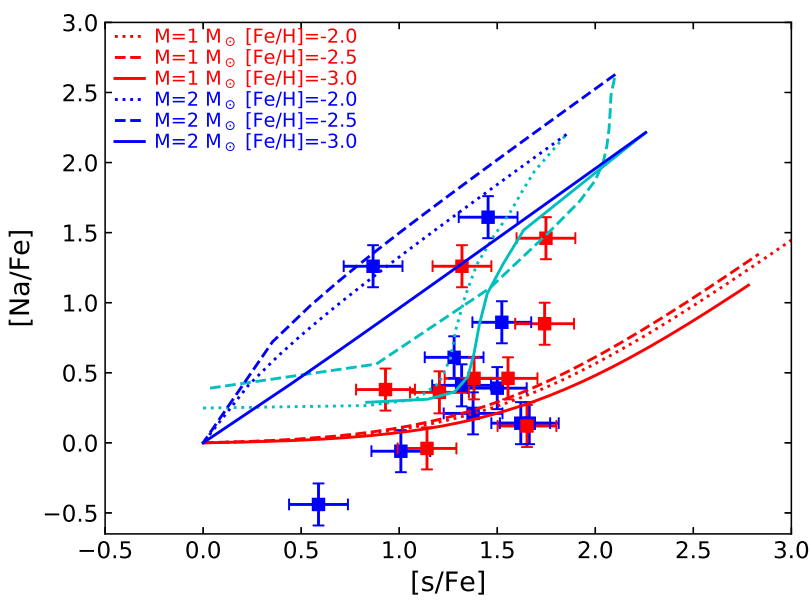

Fig. 19. Abundances of $[\mathrm{Na} / \mathrm{Fe}]$ shown as a function of $[\mathrm{s} / \mathrm{Fe}]$, where $[\mathrm{s} / \mathrm{Fe}]$ is computed as $([\mathrm{Y} / \mathrm{Fe}]+[\mathrm{Zr} / \mathrm{Fe}]+[\mathrm{Ba} / \mathrm{Fe}]+[\mathrm{La} / \mathrm{Fe}]+[\mathrm{Ce} / \mathrm{Fe}]$ $+[\mathrm{Nd} / \mathrm{Fe}]) / 6$. The measured abundances are colour-coded as in Fig. 7 , and the model predictions as in Fig. 13.

low $[\mathrm{C} / \mathrm{N}]$ ratio of these two stars, and the fact that they do not fall above the Galactic $\log \epsilon_{\mathrm{C}}$ trend in Fig. 18, can thus probably be explained by HBB operation.

\subsection{Na and heavy elements}

Figure 19 compares the $[\mathrm{Na} / \mathrm{Fe}]$ overproduction with the surface $s$-enrichment. Stars of $1 M_{\odot}$ are expected to be less enriched in $\mathrm{Na}$ than $2 M_{\odot}$ stars, where the partial mixing of protons is known to be a favourable site for $\mathrm{Na}$ production (Goriely \& Mowlavi 2000). As seen in Fig. 19, the predictions broadly cover the region occupied by the measured abundances. However, the most Na-enriched CEMP-rs stars can hardly be explained, similarly to the less Na-rich CEMP-s stars. Within the model of the partial mixing of protons at the time of the TDU, the Na production takes place in a tiny layer above the one responsible for the $s$-process (Goriely \& Mowlavi 2000). For this reason the [Na/Fe] to $[\mathrm{s} / \mathrm{Fe}]$ correlation depends on the profile of protons mixed into the $\mathrm{C}$-rich region. The overprediction of the $\mathrm{Na}$ enrichment (Fig. 19) might be an indication that the parametrization used for the mixing of protons may not be totally adequate. More studies, involving multidimensional simulations, are needed to solve this issue.

\section{5. [ls/hs] versus $[\mathrm{Mg} / \mathrm{hs}]$}

Lugaro et al. (2012) illustrated how, in a plane built with two intrinsic axes, namely the ([ls/hs], $[\mathrm{Mg} / \mathrm{hs}])$ plane (i.e. where both axes are dominated by in situ nucleosynthetic processes rather than by the chemical evolution of the Galaxy), CEMP-s and CEMP-rs stars occupy different locations.

We note, however, that in such a plane, a linear correlation of slope 1 is expected since

$[\mathrm{ls} / \mathrm{hs}]=[\mathrm{Mg} / \mathrm{hs}]+\log (\mathrm{ls} / \mathrm{Mg})-\log (\mathrm{ls} / \mathrm{Mg})_{\odot}$,

except if there is a star-to-star variation of [1s/Mg].

Nevertheless, in both Fig. 7 of Lugaro et al. (2012) and in the bottom panel of Fig. 20, CEMP-rs stars seem to cluster at lower $[\mathrm{ls} / \mathrm{hs}]$ and $[\mathrm{Mg} / \mathrm{hs}]$ ratios than CEMP-s stars. One might wonder whether this effect is merely due to the higher $[\mathrm{hs} / \mathrm{Fe}]$ of CEMP-rs stars or to a difference in $[\mathrm{Mg} / \mathrm{Fe}]$. 

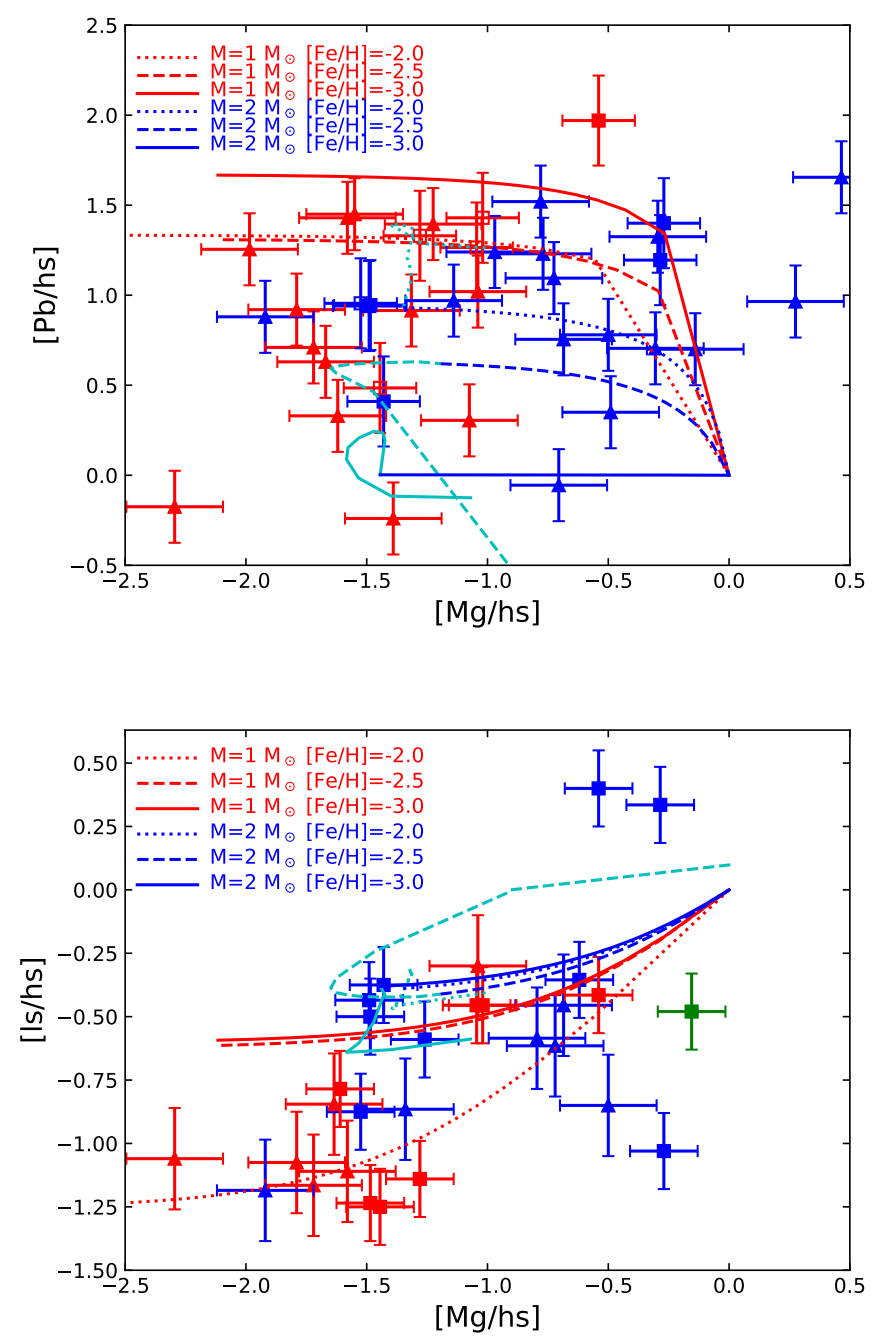

Fig. 20. Abundances $[\mathrm{Pb} / \mathrm{hs}]$ and $[\mathrm{ls} / \mathrm{hs}]$ as a function of $[\mathrm{Mg} / \mathrm{hs}]$, where $[1 \mathrm{~s}]=1 / 2([\mathrm{Y} / \mathrm{Fe}]+[\mathrm{Zr} / \mathrm{Fe}])$ and $[\mathrm{hs} / \mathrm{Fe}]=1 / 2([\mathrm{La} / \mathrm{Fe}]+[\mathrm{Ce} / \mathrm{Fe}])$. The measured abundances are colour-coded as in Fig. 7, empty squares as in Fig. 15, and the model predictions as in Fig. 13. Blue and red triangles represent CEMP-s and CEMP-rs stars from the literature.

From a theoretical point of view, CEMP-s and CEMP-rs stars can be expected to behave as shown in Fig. 20: (i) Mg-low CEMP-s stars, where $s$-process proceeds with the ${ }^{13} \mathrm{C}(\alpha, \mathrm{n}){ }^{16} \mathrm{O}$ neutron source activated in radiative conditions during the interpulse in low-mass stars; (ii) Mg-high CEMP-s stars, where the ${ }^{22} \mathrm{Ne}(\alpha, \mathrm{n})^{25} \mathrm{Mg}$ neutron source also contributes to the $s$-process nucleosynthesis during the convective thermal pulses; and (iii) Mg-low CEMP-rs stars that activate ${ }^{13} \mathrm{C}(\alpha, \mathrm{n}){ }^{16} \mathrm{O}$ in convective conditions during proton ingestion episodes in the pulses.

However, in our sample we could not find any difference in the $[\mathrm{Mg} / \mathrm{Fe}]$ ratios of CEMP-s and CEMP-rs stars (Fig. 21). We conclude that Fig. 7 of Lugaro et al. (2012) and our Fig. 21 illustrate the difference in $[\mathrm{hs} / \mathrm{Fe}]$ of CEMP-s and CEMP-rs stars, as already demonstrated in Sect. 8.1.

Globally, model predictions are in fair agreement with observations, as shown in Fig. 20. Models predict higher $[\mathrm{Pb} / \mathrm{hs}]$ and lower $[1 \mathrm{~s} / \mathrm{hs}]$ overabundances for the $1 M_{\odot}$ AGB stars relative to the $2 M_{\odot}$ ones, though in both cases $[\mathrm{Mg} / \mathrm{hs}]$ ratios span a large range typically between -2 and 0 dex. We note that the predictions regarding the light $s$-elements [1s] vary quite significantly if we adopt $\mathrm{Y}$ or $\mathrm{Zr}$ as the proxy, as seen in Fig. 13.

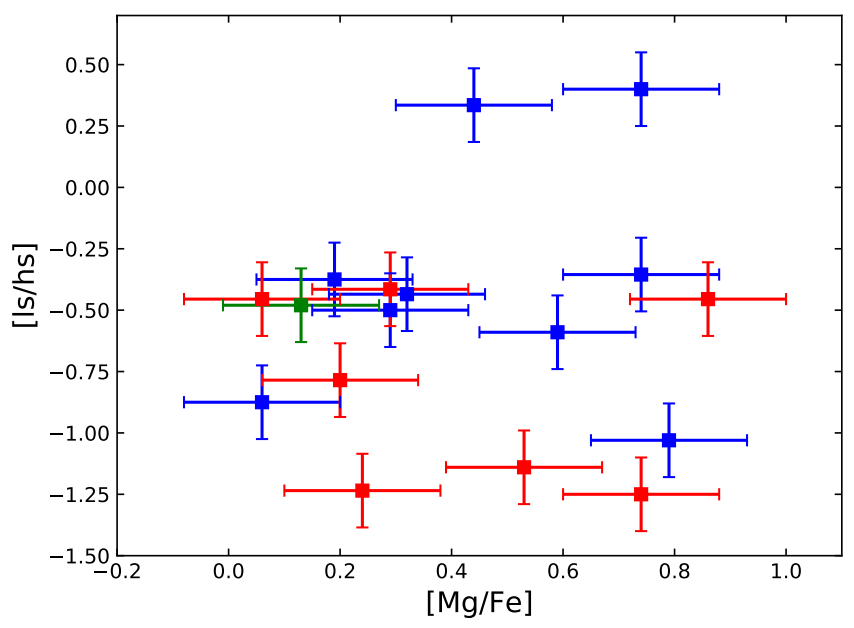

Fig. 21. $[\mathrm{Mg} / \mathrm{Fe}]$ scatter plot of CEMP-s and CEMP-rs stars. Symbols are as in Fig. 7.

\subsection{The $\mathrm{Nb}-\mathrm{Zr}$ thermometer}

$\mathrm{The} \mathrm{Nb} / \mathrm{Zr}$ thermometer offers an interesting diagnostic, allowing us to constrain the nucleosynthesis operation temperature and thus the stellar mass. In extrinsic stars, ${ }^{93} \mathrm{Zr}$ produced by the $s$-process has had time to decay (since it was produced in the companion star long ago) into mono-isotopic ${ }^{93} \mathrm{Nb}$. Therefore, $\mathrm{Nb} / \mathrm{Zr}$ ratio in extrinsic stars is equivalent to the ${ }^{93} \mathrm{Zr} / \mathrm{Zr}$ isotopic ratio in intrinsic AGB stars, which in turn probes the $s$ process operation temperature. This diagnostic has been investigated in extrinsic S stars of near solar metallicity (Neyskens et al. 2015; Shetye et al. 2018) and barium stars of sub-solar metallicity (Karinkuzhi et al. 2018). These studies have provided a new confirmation that at such moderate metallicities the ${ }^{13} \mathrm{C}$ neutron source is operating in low-mass stars. We hoped to apply this thermometer to CEMP-rs stars to constrain the putative $i$ process, its neutron source $\left({ }^{22} \mathrm{Ne}\right.$ or $\left.{ }^{13} \mathrm{C}\right)$, and its site. Unfortunately, we could not measure $\mathrm{Nb}$ abundances in most objects since at the metallicity and temperature ranges of our programme stars $\mathrm{Nb}$ is mostly ionized, and all the sensitive $\mathrm{Nb}$ II lines are located below $4000 \AA$ where HERMES spectra generally have a poor signal-to-noise ratio. This prevented us from drawing a robust conclusion about the behaviour of CEMP stars in the $\mathrm{Nb}-\mathrm{Zr}$ plane. Nevertheless, Fig. 22 shows that the two CEMP-s stars for which a niobium abundance could be safely derived follow the trend of extrinsic S stars and barium stars, as expected. The three CEMP-rs stars with measured $\mathrm{Nb}$ abundances also lie within the extrinsic-star region, and have a slightly higher $[\mathrm{Nb} / \mathrm{Fe}]$ ratio than CEMP-s stars for a given $[\mathrm{Zr} / \mathrm{Fe}]$. However, this trend should be validated by a larger sample of niobium measurements in CEMP-rs objects.

\section{HR diagram}

In this section the programme stars are located in the HR diagram using Gaia DR2 parallaxes (Gaia Collaboration 2018). The Bayesian estimates of the distances is taken from Bailer-Jones et al. (2018). STAREVOL (Siess \& Arnould 2008) evolutionary tracks using a metallicity scaled from the Asplund et al. (2009) solar mixture are overplotted from the Hertzsprung gap to the RGB tip. When the evolutionary time exceeds the Hubble time, the tracks are truncated.

Figure 23 displays the resulting HR diagrams. While the luminosities of CEMP-rs stars are compatible with the RGB 


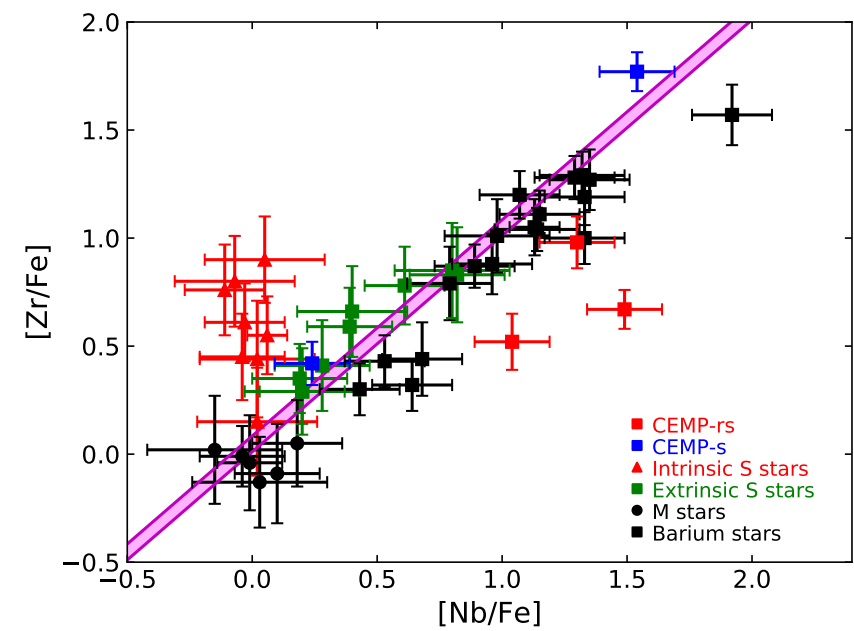

Fig. 22. $\mathrm{Nb}-\mathrm{Zr}$ plane for CEMP-s and -rs stars with measurable $\mathrm{Nb}$ abundances, along with barium stars and intrinsic or extrinsic S-type stars from Karinkuzhi et al. (2018) and Neyskens et al. (2015), respectively. The magenta shaded band indicates the expected location of stars polluted by material resulting from the $s$-process operating at temperatures between 1.0 (upper line) and $3.0 \times 10^{8} \mathrm{~K}$ (lower line). These simple predictions assume that the chain of neutron captures along the $\mathrm{Zr}$ isotopes is in local equilibrium, as explained in Karinkuzhi et al. (2018).
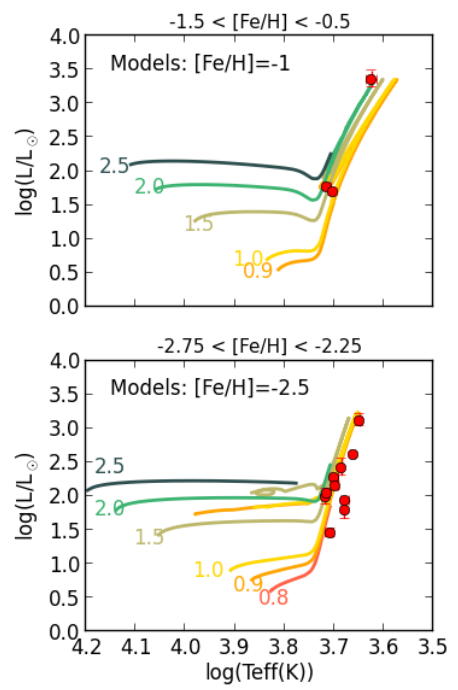

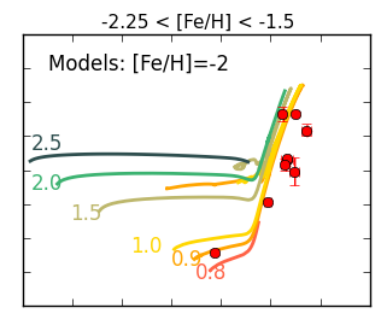

$-3.5<[\mathrm{Fe} / \mathrm{H}]<-2.75$

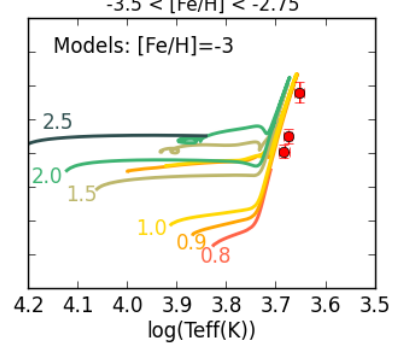

Fig. 23. HR diagrams of the programme stars (red dots) split into four metallicity bins, as labelled at the top of each subpanel. Stars with a lower limit on their luminosity are marked with an arrow. STAREVOL (Hertzsprung gap and RGB) evolutionary tracks are overplotted; their metallicity is indicated in each subpanel. The (initial) stellar masses are indicated in colour beside the corresponding track.

phase of evolution (except for CS 22887-048, which is in the Hertzsprung gap), the derived temperatures (respectively luminosities) seem too cool (respectively too low) with respect to track temperatures (respectively luminosities) for metallicities $[\mathrm{Fe} / \mathrm{H}]<-1.5$.

To reach a better agreement, $0.9 M_{\odot}$ stellar-evolution models with a different initial $\mathrm{C}$ and $\mathrm{O}$ composition were computed. Indeed CEMP stars are enriched in carbon and sometime have excess oxygen $([\mathrm{O} / \mathrm{Fe}]>0$, as a result of Galactic chemical evolution), which impacts the photospheric opacities and thus the model effective temperature. When we only change
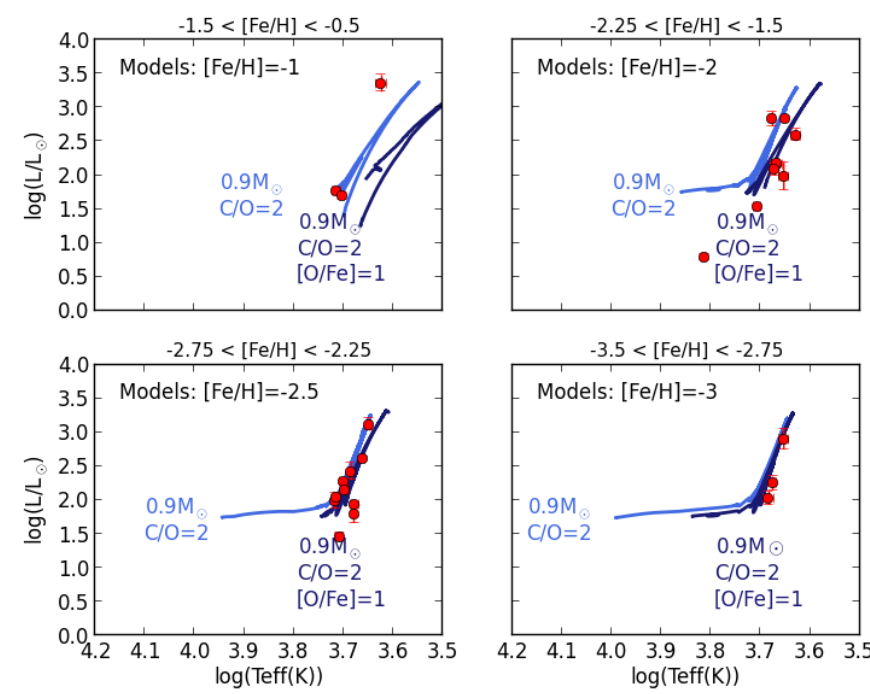

$-3.5<[\mathrm{Fe} / \mathrm{H}]<-2.75$

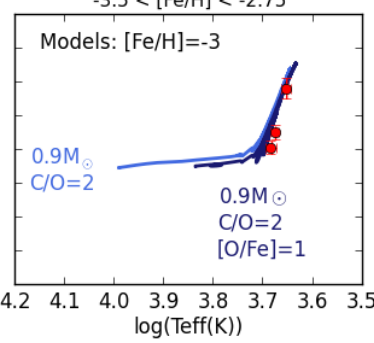

Fig. 24. Same as Fig. 23, but for $0.9 M_{\odot}$ STAREVOL evolutionary tracks of modified composition, as indicated.
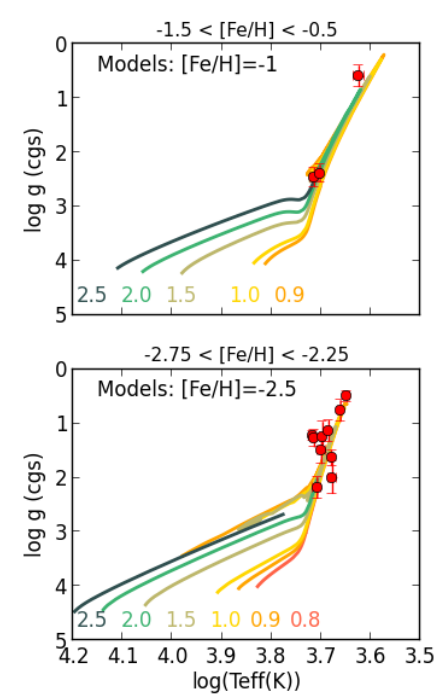

$-2.25<[\mathrm{Fe} / \mathrm{H}]<-1.5$

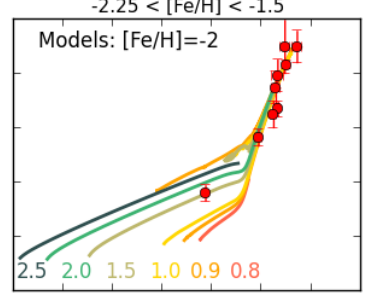

$-3.5<[\mathrm{Fe} / \mathrm{H}]<-2.75$

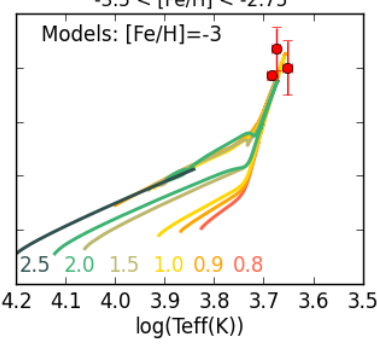

Fig. 25. Same as Fig. 23, but for the $\left(\log g, \log T_{\text {eff }}\right)$ diagram with the same STAREVOL evolutionary tracks.

the $\mathrm{C} / \mathrm{O}$ ratio, we keep the initial $\mathrm{O}$ abundance and increase that of carbon to reach the desired $\mathrm{C} / \mathrm{O}$ value. The mass fractions are then renormalized and the excess material removed from the most abundant species. When both $[\mathrm{O} / \mathrm{Fe}]$ and $\mathrm{C} / \mathrm{O}$ are imposed, we first set the $\mathrm{O}$ mass fraction to the desired value, then the carbon to get the required $\mathrm{C} / \mathrm{O}$ ratio and renormalized as before. Our CEMP-s and -rs stars have on average $\mathrm{C} / \mathrm{O}=2.4$ and $[\mathrm{O} / \mathrm{Fe}]=0.96$. The comparison between Figs. 23 and 24 illustrates how the RGB is shifted towards lower temperatures when $\mathrm{C} / \mathrm{O}=2$, and even further when an oxygen abundance of $[\mathrm{O} / \mathrm{Fe}]=1$ is adopted. In this way the agreement with the location of CEMP-rs stars in the HR diagram is improved. For completeness, the $\left(\log g, \log T_{\text {eff }}\right)$ diagrams, which are independent of distance and extinction, are also presented in Figs. 25 and 26. These examples illustrate the importance of computing stellar evolution models with the proper chemical composition, especially when considering extrinsic stars that have been substantially polluted by material with a non-solar composition. Given the degeneracy of the evolutionary tracks along the RGB, no firm conclusion can be drawn on the stellar masses. 


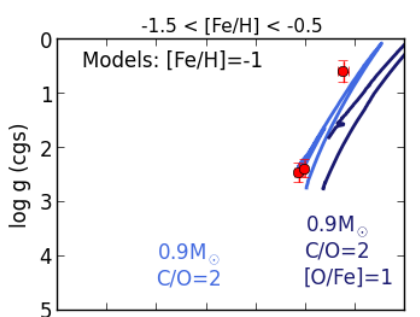

$-2.75<[\mathrm{Fe} / \mathrm{H}]<-2.25$

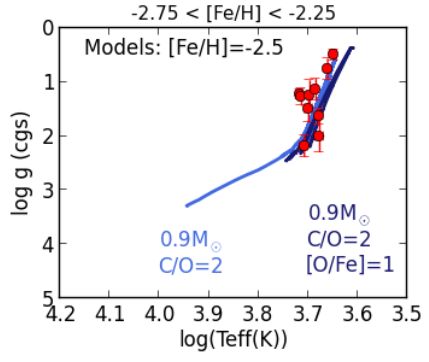

$-2.25<[\mathrm{Fe} / \mathrm{H}]<-1.5$

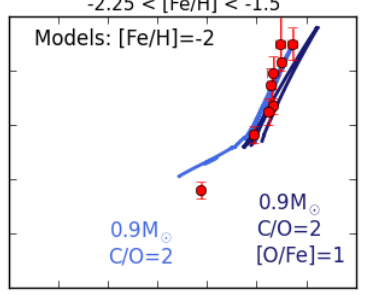

$-3.5<[\mathrm{Fe} / \mathrm{H}]<-2.75$

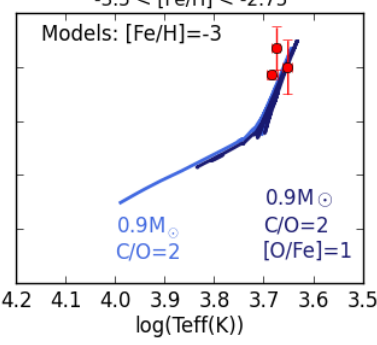

Fig. 26. Same as Fig. 25, but with the STAREVOL evolutionary tracks of modified composition used in Fig. 24.

\section{Conclusions}

The homogeneous analysis of a sample of 13 CEMP-s stars, 11 CEMP-rs stars, and one $r$-process-enriched star has shown that:

1. Using several elemental $s$ - and $r$-process abundances is important to properly classify stars as either CEMP-s or CEMP-rs, given the abundance uncertainties that can affect individual determinations. We propose here a classification based on an "abundance distance" to the $r$-process abundance profile (assumed to be universal), based on eight $s$ - and $r$-process elements.

2. Whereas it is relatively easy to isolate $r$-process-enriched objects, it is far less obvious to draw a clear separation between CEMP-rs and CEMP-s stars. This difficulty is strengthened by the recent abundance studies (including ours) measuring $r$-process abundances in stars presenting a low $s$-process enrichment. These studies have uncovered a growing number of CEMP-rs stars characterized by modest overabundance levels ${ }^{2}$. Distinguishing, at low metallicities, the origin of a $r$-contribution explained either as the star's pristine composition or as a consequence of mass transfer from an AGB having experienced proton-ingestion, will represent a challenge for future abundance studies.

3. The measured abundances of CEMP-rs stars can be reproduced by models of low-metallicity, low-mass stars experiencing a proton ingestion during a thermal pulse, yielding the intermediate neutron densities required for the $i$-process. We show that the quality of the fit is as good as that obtained when $s$-process predictions are compared to CEMP-s abundance profiles. Since there is no doubt that the $s$-process nucleosynthesis takes place in low- and intermediate-mass TP-AGB stars (e.g. because of technetium detection), similarly there should be no doubt that the $i$-process responsible for the abundance peculiarities of CEMP-rs stars could also take place in low-mass, low-metalliticy AGB stars. We therefore propose to call them CEMP-sr stars instead, since they represent a particular manifestation of the $s$-process at lowmetallicities.

4. It is important to use evolutionary tracks with a chemical composition matching that of extrinsic stars, in particular

2 CEMP-s stars with low enrichment levels also exist. with the correct $[\mathrm{C} / \mathrm{Fe}]$ and $[\mathrm{O} / \mathrm{Fe}]$ ratios, to correctly reproduce the temperatures of CEMP stars in the HR diagram. Most CEMP-rs stars are found to lie on the RGB; since they have a large binary fraction (at least $82 \%$ in our sample), they stand at the low-metallicity tail of the extrinsic-star family, with CEMP-s, $\mathrm{CH}, \mathrm{Ba}$, and extrinsic $\mathrm{S}$ stars as the other members of that family (along a sequence of increasing metallicity).

Acknowledgements. We thank the anonymous referee for useful suggestions. D.K. acknowledges the financial support from the Science and Engineering Research Board (SERB), DST, India through file number PDF/2017/002338, and CSIR-India through file No.13(9086-A)2019-Pool. A visiting research fellowship from F.R.S.-FNRS (Belgium) is also gratefully acknowledged. SVE thanks the Fondation ULB for its support. LS and SG are senior FNRS research associates. T.Me. is supported by a grant from the Fondation ULB. T.Ma. acknowledges support from MINECO under grant AYA-2017-88254-P. The Mercator telescope is operated thanks to grant number G.0C31.13 of the FWO under the "Big Science" initiative of the Flemish governement. Based on observations obtained with the HERMES spectrograph, supported by the Fund for Scientific Research of Flanders (FWO), the Research Council of K.U.Leuven, the Fonds National de la Recherche Scientifique (F.R.S.- FNRS), Belgium, the Royal Observatory of Belgium, the Observatoire de Genève, Switzerland and the Thüringer Landessternwarte Tautenburg, Germany. This research has made use of the SIMBAD database, operated at CDS, Strasbourg, France and NASA ADS, USA.

\section{References}

Abate, C., Stancliffe, R. J., \& Liu, Z.-W. 2016, A\&A, 587, A50

Alonso, A., Arribas, S., \& Martinez-Roger, C. 1996, A\&AS, 117, 227

Alonso, A., Arribas, S., \& Martínez-Roger, C. 1999, A\&AS, 139, 335

Alvarez, R., \& Plez, B. 1998, A\&A, 330, 1109

Amarsi, A. M., Asplund, M., Collet, R., \& Leenaarts, J. 2016, MNRAS, 455, 3735

Andrievsky, S. M., Spite, M., Korotin, S. A., et al. 2009, A\&A, 494, 1083

Andrievsky, S. M., Spite, F., Korotin, S. A., et al. 2011, A\&A, 530, A105

Aoki, W., Ryan, S. G., Norris, J. E., et al. 2002, ApJ, 580, 1149

Aoki, W., Beers, T. C., Christlieb, N., et al. 2007, ApJ, 655, 492

Asplund, M., Grevesse, N., Sauval, A. J., Allende Prieto, C., \& Kiselman, D. 2005, A\&A, 435, 339

Asplund, M., Grevesse, N., Sauval, A. J., \& Scott, P. 2009, ARA\&A, 47, 481

Bailer-Jones, C. A. L., Rybizki, J., Fouesneau, M., Mantelet, G., \& Andrae, R. 2018, AJ, 156, 58

Barbuy, B., Cayrel, R., Spite, M., et al. 1997, A\&A, 317, L63

Beers, T. C., \& Christlieb, N. 2005, ARA\&A, 43, 531

Beers, T. C., Preston, G. W., \& Shectman, S. A. 1992, AJ, 103, 1987

Bergemann, M., Hansen, C. J., Bautista, M., \& Ruchti, G. 2012, A\&A, 546, A90

Bisterzo, S., Gallino, R., Straniero, O., Cristallo, S., \& Käppeler, F. 2011, MNRAS, 418, 284

Boothroyd, A. I., Sackmann, I.-J., \& Wasserburg, G. J. 1995, ApJ, 442, L21

Campbell, S., Lugaro, M., \& Karakas, A. 2010, A\&A, 522, L6

Choplin, A., Siess, L., \& Goriely, S. 2021, A\&A, submitted

Christlieb, N., Green, P. J., Wisotzki, L., \& Reimers, D. 2001, A\&A, 375, 366

Clarkson, O., Herwig, F., \& Pignatari, M. 2018, MNRAS, 474, L37

Cowan, J. J., \& Rose, W. K. 1977, ApJ, 212, 149

Denissenkov, P. A., Herwig, F., Battino, U., et al. 2017, ApJ, 834, L10

Denissenkov, P., Herwig, F., Woodward, P., et al. 2018, MNRAS, 448, 4258

De Smedt, K., Van Winckel, H., Kamath, D., et al. 2016, A\&A, 587, A6

Gaia Collaboration (Brown, A. G. A., et al.) 2018, A\&A, 616, A1

Goriely, S. 1999, A\&A, 342, 881

Goriely, S., \& Arnould, M. 1997, A\&A, 322, L29

Goriely, S., \& Mowlavi, N. 2000, A\&A, 362, 599

Goriely, S., \& Siess, L. 2005, in From Lithium to Uranium: Elemental Tracers of Early Cosmic Evolution, eds. V. Hill, P. Francois, \& F. Primas, IAU Symp., 228,451

Goriely, S., \& Siess, L. 2018, A\&A, 609, A29

Gorlova, N., Van Winckel, H., Vos, J., et al. 2014, ArXiv e-prints [arXiv:1403.2287]

Goswami, A., \& Aoki, W. 2010, MNRAS, 404, 253

Goswami, A., Aoki, W., Beers, T. C., et al. 2006, MNRAS, 372, 343

Goswami, A., Karinkuzhi, D., \& Shantikumar, N. S. 2010, MNRAS, 402, 111

Goswami, A., Aoki, W., \& Karinkuzhi, D. 2016, MNRAS, 455, 402

Gull, M., Frebel, A., Cain, M. G., et al. 2018, ApJ, 862, 174 
Gustafsson, B., Edvardsson, B., Eriksson, K., et al. 2008, A\&A, 486, 951 Hampel, M., Stancliffe, R. J., Lugaro, M., \& Meyer, B. S. 2016, ApJ, 831, 171 Hansen, T. T., Andersen, J., Nordström, B., et al. 2015, A\&A, 583, A49 Hansen, T. T., Andersen, J., Nordström, B., et al. 2016a, A\&A, 588, A3 Hansen, C. J., Nordström, B., Hansen, T. T., et al. 2016b, A\&A, 588, A37 Hansen, T. T., Andersen, J., Nordström, B., et al. 2016c, A\&A, 586, A160 Heiter, U., Lind, K., Asplund, M., et al. 2015, Phys. Scr., 90, 054010

Heiter, U., Lind, K., Bergemann, M., et al. 2021, A\&A, in press, https : //doi org/10.1051/0004-6361/201936291

Herwig, F., Pignatari, M., Woodward, P. R., et al. 2011, ApJ, 727, 89

Hill, V., Barbuy, B., Spite, M., et al. 2000, A\&A, 353, 557

Hollek, J. K., Frebel, A., Placco, V. M., et al. 2015, ApJ, 814, 121

Ivans, I. I., Simmerer, J., Sneden, C., et al. 2006, ApJ, 645, 613

Iwamoto, N., Kajino, T., Mathews, G., Fujimoto, M., \& Aoki, W. 2004, ApJ, 602,377

Johnson, J. A. 2002, ApJS, 139, 219

Johnson, J. A., Herwig, F., Beers, T. C., \& Christlieb, N. 2007, ApJ, 658, 1203

Jones, S., Ritter, C., Herwig, F., et al. 2016, MNRAS, 455, 3848

Jonsell, K., Barklem, P. S., Gustafsson, B., et al. 2006, A\&A, 451, 651

Jorissen, A., Začs, L., Udry, S., Lindgren, H., \& Musaev, F. A. 2005, A\&A, 441, 1135

Jorissen, A., Van Eck, S., Van Winckel, H., et al. 2016, A\&A, 586, A158

Jorissen, A., Boffin, H. M. J., Karinkuzhi, D., et al. 2019, A\&A, 626, A127

Karinkuzhi, D., \& Goswami, A. 2014, MNRAS, 440, 1095

Karinkuzhi, D., \& Goswami, A. 2015, MNRAS, 446, 2348

Karinkuzhi, D., Van Eck, S., Jorissen, A., et al. 2018, A\&A, 618, A32

Keenan, P. C. 1942, ApJ, 96, 101

Kennedy, C. R., Sivarani, T., Beers, T. C., et al. 2011, AJ, 141, 102

Lodders, K. 2003, ApJ, 591, 1220

Lucatello, S., Tsangarides, S., Beers, T. C., et al. 2005, ApJ, 625, 825

Lugaro, M., Karakas, A. I., Stancliffe, R. J., \& Rijs, C. 2012, ApJ, 747, 2

Lugaro, M., Campbell, S. W., Van Winckel, H., et al. 2015, A\&A, 583, A77

Mashonkina, L., \& Christlieb, N. 2014, A\&A, 565, A123

Mashonkina, L., \& Gehren, T. 2000, A\&A, 364, 249

Mashonkina, L., Gehren, T., \& Bikmaev, I. 1999, A\&A, 343, 519
Mashonkina, L., Zhao, G., Gehren, T., et al. 2008, A\&A, 478, 529 Mashonkina, L., Ryabtsev, A., \& Frebel, A. 2012, A\&A, 540, A98 Masseron, T., Van Eck, S., Famaey, B., et al. 2006, A\&A, 455, 1059 Masseron, T., Johnson, J. A., Plez, B., et al. 2010, A\&A, 509, A93

Masseron, T., Merle, T., \& Hawkins, K. 2016, Astrophysics Source Code Library [record ascl:1605.004]

McClure, R. D., \& Woodsworth, A. W. 1990, ApJ, 352, 709

Neyskens, P., Van Eck, S., Jorissen, A., et al. 2015, Nature, 517, 174

Pereira, C. B., Sales Silva, J. V., Chavero, C., Roig, F., \& Jilinski, E. 2011, A\&A, 533, A51

Pereira, C. B., Drake, N. A., \& Roig, F. 2019, MNRAS, 488, 482

Placco, V. M., Beers, T. C., Ivans, I. I., et al. 2015, ApJ, 812, 109

Plez, B. 2012, Astrophysics Source Code Library [record ascl:1205 . 004]

Preston, G. W., \& Sneden, C. 2001, AJ, 122, 1545

Ramírez, I., \& Meléndez, J. 2004, ApJ, 609, 417

Raskin, G., van Winckel, H., Hensberge, H., et al. 2011, A\&A, 526, A69

Reimers, D. 1975, Mem. Soc. R. Sci. Liege, 8, 369

Reyniers, M., \& Van Winckel, H. 2007, A\&A, 463, L1

Roederer, I. 2011, ApJ, 732, L17

Roederer, I. U., Cowan, J. J., Karakas, A. I., et al. 2010, ApJ, 724, 975

Shetye, S., Van Eck, S., Jorissen, A., et al. 2018, A\&A, 620, A148

Shetye, S., Goriely, S., Siess, L., et al. 2019, A\&A, 625, L1

Siess, L., \& Arnould, M. 2008, A\&A, 489, 395

Sneden, C., Roederer, I., Cowan, J., \& Lawler, J. E. 2010, Nuclei in the Cosmos, 74

Spite, M., Caffau, E., Bonifacio, P., et al. 2013, A\&A, 552, A107

Starkenburg, E., Shetrone, M. D., McConnachie, A. W., \& Venn, K. A. 2014, MNRAS, 441, 1217

Tsangarides, S. A. 2005, PhD Thesis, Open University

Van Eck, S., Goriely, S., Jorissen, A., \& Plez, B. 2003, A\&A, 404, 291

Vanture, A. D. 1992a, AJ, 104, 1997

Vanture, A. D. 1992b, AJ, 103, 2035

Vanture, A. D., Wallerstein, G., Gallino, R., \& Masera, S. 2003, ApJ, 587, 384

Vassiliadis, E., \& Wood, P. R. 1993, ApJ, 413, 641

Yoon, J., Beers, T. C., Placco, V. M., et al. 2016, ApJ, 833, 20 


\section{Appendix A: Line list}

Table A.1. Lines used in the abundance analysis.

\begin{tabular}{lcr}
\hline \hline $\begin{array}{l}\lambda \\
(\AA)\end{array}$ & $\begin{array}{l}\chi_{\text {low }} \\
(\mathrm{eV})\end{array}$ & $\log g f$ \\
\hline $\mathrm{OI}$ & & \\
6300.304 & 0.000 & -9.715 \\
7771.941 & 9.146 & 0.369 \\
7774.161 & 9.146 & 0.223 \\
7775.338 & 9.146 & 0.001 \\
\hline $\mathrm{Na} \mathrm{I}$ & & \\
5682.633 & 2.102 & -0.706 \\
5688.205 & 2.104 & -0.450 \\
6154.226 & 2.102 & -1.547 \\
6160.747 & 2.104 & -1.246
\end{tabular}

Notes. The full table is only available at the CDS.

\section{Appendix B: Elemental abundances for the programme stars}

Table B.1. Light element abundances.

\begin{tabular}{lrrrrrrrrr}
\hline \hline Star name & {$[\mathrm{Ca} / \mathrm{Fe}]$} & {$[\mathrm{Sc} / \mathrm{Fe}]$} & {$[\mathrm{Ti} / \mathrm{Fe}]$} & {$[\mathrm{Cr} / \mathrm{Fe}]$} & {$[\mathrm{Mn} / \mathrm{Fe}]$} & {$[\mathrm{Co} / \mathrm{Fe}]$} & {$[\mathrm{Ni} / \mathrm{Fe}]$} & {$[\mathrm{Cu} / \mathrm{Fe}]$} & {$[\mathrm{Zn} / \mathrm{Fe}]$} \\
\hline CS 22887-048 & 0.66 & - & 0.45 & 0.16 & -0.33 & 0.11 & 0.18 & - & 0.39 \\
CS 22891-171 & 0.81 & 0.10 & 0.69 & -0.09 & -0.68 & 0.06 & 0.43 & - & 0.29 \\
CS 22942-019 & 0.31 & 0.35 & 0.40 & -0.14 & -0.23 & 0.11 & 0.18 & - & 0.24 \\
CS 30322-023 & 0.36 & - & 0.43 & -0.39 & -0.33 & -0.04 & 0.13 & - & 0.34 \\
HD 26 & 0.19 & 0.38 & 0.63 & 0.07 & 0.05 & - & 0.11 & 0.09 & 0.42 \\
HD 5223 & 0.31 & 0.07 & 0.05 & -0.34 & -0.43 & - & 0.08 & 0.11 & 0.14 \\
HD 55496 & 0.74 & 0.31 & 0.39 & -0.12 & -0.32 & 0.12 & 0.18 & 0.32 & 0.74 \\
HD 76396 & 0.53 & 0.55 & 0.37 & 0.13 & -0.36 & 0.28 & 0.15 & 0.18 & 0.56 \\
HD 145777 & 0.58 & 0.02 & 0.04 & -0.32 & -0.33 & - & -0.45 & - & -0.04 \\
HD 187861 & 0.49 & 0.60 & 0.50 & -0.19 & - & - & 0.08 & $0.81:$ & 0.04 \\
HD 196944 & 0.36 & 0.05 & 0.15 & -0.22 & -0.38 & -0.09 & -0.02 & - & 0.24 \\
HD 198269 & 0.38 & 0.25 & 0.15 & -0.38 & -0.51 & 0.11 & -0.17 & -0.09 & 0.04 \\
HD 201626 & 0.55 & 0.30 & 0.12 & 0.13 & -0.06 & -0.52 & 0.02 & -0.02 & 0.21 \\
HD 206983 & -0.35 & -0.26 & 0.04 & -0.55 & -0.84 & - & -0.93 & - & - \\
HD 209621 & 0.51 & 0.70 & 0.57 & -0.04 & - & -0.39 & -0.02 & -0.19 & 0.24 \\
HD 221170 & 0.33 & 0.08 & 0.15 & -0.14 & -0.33 & -0.19 & -0.07 & 0.21 & 0.09 \\
HD 224959 & 0.42 & 0.21 & 0.29 & 0.07 & -0.47 & -0.03 & 0.14 & -0.43 & -0.05 \\
HE 0111-1346 & 0.55 & 0.25 & 0.15 & -0.54 & - & - & - & 0.21 & 0.24 \\
HE 0151-0341 & 0.55 & 0.57 & 0.34 & -0.20 & -0.54 & -0.40 & 0.07 & - & 0.33 \\
HE 0319-0215 & 0.57 & 0.25 & 0.09 & 0.08 & -0.38 & - & 0.08 & - & 0.34 \\
HE 0507-1653 & 0.67 & 0.20 & 0.32 & -0.05 & -0.20 & 0.16 & -0.11 & -0.14 & 0.09 \\
HE 1120-2122 & 0.18 & 0.15 & -0.16 & -0.36 & -0.43 & - & -0.27 & - & -0.01 \\
HE 1429-0551 & 0.36 & - & 0.29 & -0.17 & -0.43 & 0.11 & -0.02 & 0.51 & 0.14 \\
HE 2144-1832 & 0.39 & 0.55 & 0.50 & 0.04 & -0.05 & - & -0.02 & 0.03 & 0.39 \\
HE 2255-1724 & 0.49 & 0.35 & 0.22 & -0.03 & -0.26 & 0.03 & - & -0.47 & 0.26 \\
\hline
\end{tabular}


Table B.2. Elemental abundances.

\begin{tabular}{|c|c|c|c|c|c|c|c|c|}
\hline & \multirow[b]{2}{*}{$Z$} & \multirow[b]{2}{*}{$\log _{\odot} \epsilon^{(a)}$} & \multicolumn{3}{|c|}{ CS 22891-171 } & \multicolumn{3}{|c|}{ CS 22887-048 } \\
\hline & & & $\log \epsilon$ & $\sigma_{1}(\mathrm{~N})$ & {$[\mathrm{X} / \mathrm{Fe}] \pm \sigma_{\mathrm{t}}$} & $\log \epsilon$ & $\sigma_{1}(\mathrm{~N})$ & {$[\mathrm{X} / \mathrm{Fe}] \pm \sigma_{\mathrm{t}}$} \\
\hline $\mathrm{C}$ & 6 & 8.43 & 8.00 & $0.08(3)$ & $2.07 \pm 0.07$ & 8.20 & $0.08(3)$ & $1.87 \pm 0.06$ \\
\hline${ }^{12} \mathrm{C} /{ }^{13} \mathrm{C}$ & - & - & - & - & 6 & - & - & 12 \\
\hline $\mathrm{N}$ & 7 & 7.83 & 7.00 & $0.08(5)$ & $1.67 \pm 0.33$ & 7.40 & $0.05(5)$ & $1.67 \pm 0.32$ \\
\hline $\mathrm{O}$ & 8 & 8.69 & - & - & - & - & - & - \\
\hline $\mathrm{Na}$ & 11 & 6.24 & 5.20 & $0.05(2)$ & $1.46 \pm 0.11$ & - & - & - \\
\hline $\mathrm{Mg}$ & 12 & 7.60 & 5.90 & $0.10(1)$ & $0.74 \pm 0.10$ & 6.15 & $0.10(1)$ & $0.59 \pm 0.09$ \\
\hline $\mathrm{Fe}$ & 26 & 7.50 & 5.00 & $0.12(18)$ & - & 5.40 & $0.06(32)$ & - \\
\hline $\mathrm{Sr}_{\text {LTE }}$ & 38 & 2.87 & - & - & - & 2.35 & $0.05(1)$ & $1.58 \pm 0.13$ \\
\hline $\mathrm{Sr}_{\mathrm{NLTE}}$ & 38 & 2.87 & - & - & - & 2.30 & $0.05(1)$ & $1.53 \pm 0.13$ \\
\hline$Y^{\text {NLIE }}$ & 39 & 2.21 & 0.50 & $0.03(3)$ & $0.90 \pm 0.13$ & 1.00 & $0.05(2)$ & $0.89 \pm 0.13$ \\
\hline $\mathrm{Zr}$ & 40 & 2.58 & 1.05 & $0.15(3)$ & $0.97 \pm 0.12$ & 2.00 & $0.08(4)$ & $1.52 \pm 0.09$ \\
\hline $\mathrm{Ba}$ & 56 & 2.18 & 1.75 & $0.10(1)$ & $2.07 \pm 0.15$ & 1.90 & $0.10(1)$ & $1.82 \pm 0.15$ \\
\hline $\mathrm{La}$ & 57 & 1.10 & 0.85 & $0.09(12)$ & $2.25 \pm 0.09$ & 0.90 & $0.08(5)$ & $1.90 \pm 0.09$ \\
\hline $\mathrm{Ce}$ & 58 & 1.58 & 1.20 & $0.14(10)$ & $2.12 \pm 0.11$ & 1.30 & $0.11(5)$ & $1.82 \pm 0.11$ \\
\hline $\operatorname{Pr}$ & 59 & 0.72 & 0.30 & $0.03(5)$ & $2.08 \pm 0.08$ & - & - & - \\
\hline $\mathrm{Nd}$ & 60 & 1.42 & 1.10 & $0.03(14)$ & $2.18 \pm 0.08$ & 1.00 & $0.04(4)$ & $1.68 \pm 0.08$ \\
\hline $\mathrm{Sm}$ & 62 & 0.96 & 0.25 & $0.07(5)$ & $1.79 \pm 0.10$ & 0.40 & $0.02(2)$ & $1.54 \pm 0.10$ \\
\hline $\mathrm{Eu}_{\mathrm{LTE}}$ & 63 & 0.52 & -0.25 & $0.15(3)$ & $1.73 \pm 0.16$ & -0.25 & $0.03(2)$ & $1.33 \pm 0.14$ \\
\hline $\mathrm{Eu}_{\mathrm{NLTE}}$ & 63 & 0.52 & -0.28 & $0.10(1)$ & $1.70 \pm 0.16$ & -0.18 & $0.10(1)$ & $1.40 \pm 0.15$ \\
\hline Gd & 64 & 1.07 & 0.30 & $0.10(1)$ & $1.73 \pm 0.17$ & - & - & - \\
\hline Dy & 66 & 1.10 & 0.00 & $0.15(2)$ & $1.40 \pm 0.15$ & - & - & - \\
\hline Er & 68 & 0.92 & 0.00 & $0.10(1)$ & $1.58 \pm 0.11$ & 1.00 & $0.10(1)$ & $2.18 \pm 0.11$ \\
\hline $\mathrm{Hf}$ & 72 & 0.85 & $0.45:$ & $0.10(1)$ & $2.10 \pm 0.10$ & - & - & - \\
\hline $\mathrm{Pb}_{\text {LTE }}$ & 82 & 1.75 & 1.40: & $0.10(1)$ & $2.15 \pm 0.24$ & - & - & - \\
\hline $\mathrm{Pb}_{\text {NLTE }}$ & 82 & 1.75 & 1.92: & $0.10(1)$ & $2.67 \pm 0.24$ & - & - & - \\
\hline
\end{tabular}

Notes. ${ }^{(a)}$ Asplund et al. (2009). Uncertain abundances due to noisy or blended region are indicated with ":". The full table is only available at the CDS. 
D. Karinkuzhi et al.: Low-mass low-metallicity AGB stars as an efficient $i$-process site explaining CEMP-rs stars

Table B.3. Abundance ratios for the programme stars.

\begin{tabular}{lrrrr}
\hline \hline Star name & {$[\mathrm{ls} / \mathrm{Fe}]$} & {$[\mathrm{hs} / \mathrm{Fe}]$} & {$[\mathrm{hs} / \mathrm{ls}]$} & {$[\mathrm{s} / \mathrm{Fe}]$} \\
\hline CS 22887-048 & 1.26 & 1.85 & 0.59 & 1.62 \\
CS 22891-171 & 0.94 & 2.19 & 1.25 & 1.75 \\
CS 22942-019 & 1.68 & 1.28 & -0.40 & 1.45 \\
CS 30322-023 & 0.03 & 1.06 & 1.03 & 0.65 \\
HD 26 & 1.38 & 1.81 & 0.43 & 1.66 \\
HD 5223 & 0.70 & 1.48 & 0.78 & 1.21 \\
HD 55496 & 1.06 & 0.73 & -0.33 & 0.87 \\
HD 76396 & 1.43 & 1.88 & 0.45 & 1.74 \\
HD 145777 & 0.65 & 1.11 & 0.46 & 0.93 \\
HD 187861 & 0.61 & 1.74 & 1.13 & 1.38 \\
HD 196944 & 0.42 & 0.83 & 0.41 & 0.72 \\
HD 198269 & 0.47 & 1.27 & 0.80 & 1.01 \\
HD 201626 & 0.94 & 1.60 & 0.66 & 1.38 \\
HD 206983 & 0.25 & 0.74 & 0.49 & 0.59 \\
HD 209621 & 1.03 & 1.81 & 0.78 & 1.56 \\
HD 221170 & -0.20 & 0.29 & 0.49 & 0.07 \\
HD 224959 & 0.74 & 2.05 & 1.31 & 1.65 \\
HE 0111-1346 & 0.85 & 1.87 & 1.02 & 1.52 \\
HE 0151-0341 & 0.67 & 1.81 & 1.14 & 1.44 \\
HE 0319-0215 & 0.49 & 1.73 & 1.24 & 1.32 \\
HE 0507-1653 & 1.28 & 1.78 & 0.50 & 1.62 \\
HE 1120-2122 & 0.75 & 1.35 & 0.60 & 1.14 \\
HE 1429-0551 & 1.01 & 1.36 & 0.35 & 1.28 \\
HE 2144-1832 & 1.25 & 1.62 & 0.37 & 1.50 \\
HE 2255-1724 & 0.71 & 1.59 & 0.88 & 1.32 \\
\hline
\end{tabular}

Notes. $\quad[\mathrm{ls} / \mathrm{Fe}]=([\mathrm{Y} / \mathrm{Fe}]+[\mathrm{Zr} / \mathrm{Fe}]) / 2, \quad[\mathrm{hs} / \mathrm{Fe}]=([\mathrm{La} / \mathrm{Fe}]+[\mathrm{Ce} / \mathrm{Fe}]) / 2$, $[\mathrm{s} / \mathrm{Fe}]=([\mathrm{Y} / \mathrm{Fe}]+[\mathrm{Zr} / \mathrm{Fe}]+[\mathrm{Ba} / \mathrm{Fe}]+[\mathrm{La} / \mathrm{Fe}]+[\mathrm{Ce} / \mathrm{Fe}]+[\mathrm{Nd} / \mathrm{Fe}]) / 6$.
Table B.4. Standard, minimum and maximum $s$ - and $r$-contributions to the Solar System abundances.

\begin{tabular}{|c|c|c|c|c|c|c|c|}
\hline$Z$ & El. & Stand $s$ & $\operatorname{Min} s$ & $\operatorname{Max} s$ & Stand $r$ & $\operatorname{Min} r$ & $\operatorname{Max} r$ \\
\hline 30 & $\mathrm{Zn}$ & 2.44 & 2.40 & 2.48 & 2.44 & 2.38 & 2.48 \\
\hline 31 & $\mathrm{Ga}$ & 3.12 & 3.10 & 3.14 & 2.46 & - & 2.81 \\
\hline 32 & $\mathrm{Ge}$ & 3.52 & 3.47 & 3.56 & 3.09 & 2.80 & 3.30 \\
\hline 33 & As & 2.35 & 2.33 & 2.37 & 2.13 & 2.06 & 2.22 \\
\hline 34 & $\mathrm{Se}$ & 3.35 & 3.33 & 3.37 & 3.13 & 3.09 & 3.28 \\
\hline 35 & $\mathrm{Br}$ & 2.61 & 2.56 & 2.65 & 2.50 & 2.15 & 2.57 \\
\hline 36 & $\mathrm{Kr}$ & 3.23 & 3.14 & 3.31 & 3.00 & 2.79 & 3.16 \\
\hline 37 & $\mathrm{Rb}$ & 2.41 & 2.39 & 2.43 & 2.05 & 1.57 & 2.25 \\
\hline 38 & $\mathrm{Sr}$ & 2.81 & 2.78 & 2.83 & 2.16 & - & 2.22 \\
\hline 39 & $\mathrm{Y}$ & 2.21 & 2.19 & 2.23 & 1.60 & - & 1.74 \\
\hline 40 & $\mathrm{Zr}$ & 2.60 & 2.58 & 2.62 & 2.01 & 1.69 & 2.15 \\
\hline 41 & $\mathrm{Nb}$ & 1.41 & 1.39 & 1.43 & 0.54 & - & 0.94 \\
\hline 42 & Mo & 1.72 & 1.70 & 1.74 & 1.27 & 1.10 & 1.42 \\
\hline 44 & $\mathrm{Ru}$ & 1.73 & 1.68 & 1.77 & 1.59 & 1.52 & 1.66 \\
\hline 45 & $\mathrm{Rh}$ & 1.09 & 1.00 & 1.17 & 1.02 & 0.87 & 1.12 \\
\hline 46 & $\mathrm{Pd}$ & 1.63 & 1.58 & 1.67 & 1.40 & 1.28 & 1.50 \\
\hline 47 & $\mathrm{Ag}$ & 1.23 & 1.19 & 1.27 & 1.13 & 1.04 & 1.20 \\
\hline 48 & $\mathrm{Cd}$ & 1.68 & 1.63 & 1.72 & 1.41 & 1.19 & 1.55 \\
\hline 49 & In & 0.78 & 0.73 & 0.82 & 0.60 & 0.46 & 0.68 \\
\hline 50 & $\mathrm{Sn}$ & 2.04 & 2.00 & 2.08 & 1.62 & 1.35 & 1.77 \\
\hline 51 & $\mathrm{Te}$ & 1.00 & 0.95 & 1.04 & 0.84 & 0.73 & 0.94 \\
\hline 52 & In & 2.18 & 2.14 & 2.22 & 2.13 & 2.07 & 2.18 \\
\hline 53 & I & 1.50 & 1.41 & 1.58 & 1.48 & 1.37 & 1.56 \\
\hline 54 & $\mathrm{Xe}$ & 2.13 & 2.04 & 2.21 & 2.06 & 1.92 & 2.16 \\
\hline 55 & $\mathrm{Cs}$ & 1.12 & 1.10 & 1.14 & 1.04 & 1.01 & 1.08 \\
\hline 56 & $\mathrm{Ba}$ & 2.17 & 2.14 & 2.19 & 1.35 & - & 1.75 \\
\hline 57 & $\mathrm{La}$ & 1.21 & 1.19 & 1.23 & 0.75 & 0.34 & 0.94 \\
\hline 58 & $\mathrm{Ce}$ & 1.63 & 1.60 & 1.65 & 0.91 & - & 1.20 \\
\hline 59 & $\operatorname{Pr}$ & 0.81 & 0.76 & 0.85 & 0.59 & 0.32 & 0.67 \\
\hline 60 & $\mathrm{Nd}$ & 1.35 & 1.33 & 1.37 & 1.12 & 1.00 & 1.19 \\
\hline 62 & $\mathrm{Sm}$ & 0.87 & 0.85 & 0.89 & 0.81 & 0.73 & 0.81 \\
\hline 63 & $\mathrm{Eu}$ & 0.55 & 0.53 & 0.57 & 0.53 & 0.41 & 0.55 \\
\hline 64 & $\mathrm{Gd}$ & 1.07 & 1.05 & 1.09 & 1.01 & 0.95 & 1.04 \\
\hline 65 & $\mathrm{~Tb}$ & 0.36 & 0.32 & 0.40 & 0.33 & 0.27 & 0.38 \\
\hline 66 & Dy & 1.15 & 1.13 & 1.17 & 1.11 & 1.06 & 1.11 \\
\hline 67 & Ho & 0.50 & 0.46 & 0.55 & 0.47 & 0.42 & 0.52 \\
\hline 68 & $\mathrm{Er}$ & 0.96 & 0.94 & 0.98 & 0.89 & 0.84 & 0.93 \\
\hline 69 & $\mathrm{Tm}$ & 0.15 & 0.10 & 0.19 & 0.08 & -0.04 & 0.14 \\
\hline 70 & $\mathrm{Yb}$ & 0.94 & 0.91 & 0.96 & 0.77 & 0.62 & 0.84 \\
\hline 71 & $\mathrm{Lu}$ & 0.12 & 0.10 & 0.14 & 0.03 & -0.25 & 0.12 \\
\hline 72 & Hf & 0.72 & 0.70 & 0.75 & 0.40 & 0.22 & 0.49 \\
\hline 73 & $\mathrm{Ta}$ & -0.15 & -0.19 & -0.11 & -0.42 & -0.66 & -0.29 \\
\hline 74 & W & 0.68 & 0.65 & 0.71 & 0.29 & -0.31 & 0.45 \\
\hline 75 & $\operatorname{Re}$ & 0.28 & 0.25 & 0.31 & 0.22 & 0.15 & 0.28 \\
\hline 76 & Os & 1.36 & 1.34 & 1.38 & 1.33 & 1.29 & 1.36 \\
\hline 77 & $\mathrm{Ir}$ & 1.35 & 1.33 & 1.36 & 1.34 & 1.32 & 1.36 \\
\hline 78 & $\mathrm{Pt}$ & 1.67 & 1.63 & 1.71 & 1.65 & 1.59 & 1.70 \\
\hline 79 & $\mathrm{Au}$ & 0.86 & 0.84 & 0.88 & 0.83 & 0.80 & 0.86 \\
\hline 80 & $\mathrm{Hg}$ & 1.12 & 1.02 & 1.20 & 0.76 & 0.40 & 0.99 \\
\hline 81 & $\mathrm{Tl}$ & 0.81 & 0.77 & 0.86 & 0.27 & - & 0.70 \\
\hline 82 & $\mathrm{~Pb}$ & 2.05 & 2.00 & 2.09 & 1.08 & 0.11 & 1.96 \\
\hline 83 & $\mathrm{Bi}$ & 0.71 & 0.64 & 0.78 & 0.25 & -0.45 & 0.76 \\
\hline
\end{tabular}

Notes. Adapted from Table 3 of Goriely (1999), here presented per element (El.) and in the scale $\log \epsilon_{x_{i}}=\log _{10}\left(n_{x_{i}} / n_{\mathrm{H}}\right)+12$. A dash means that the corresponding abundance limit is totally negligible. 